\title{
ON A LONG RANGE SEGREGATION MODEL
}

\author{
L. CAFFARELLI, S. PATRIZI, AND V. QUITALO
}

\begin{abstract}
In this work we study the properties of segregation processes modeled by a family of equations

$$
L\left(u_{i}\right)(x)=u_{i}(x) F_{i}\left(u_{1}, \ldots, u_{K}\right)(x) \quad i=1, \ldots, K
$$

where $F_{i}\left(u_{1}, \ldots, u_{K}\right)(x)$ is a non-local factor that takes into consideration the values of the functions $u_{j}$ 's in a full neighborhood of $x$. We consider as a model problem

$$
\Delta u_{i}^{\varepsilon}(x)=\frac{1}{\varepsilon^{2}} u_{i}^{\varepsilon}(x) \sum_{i \neq j} H\left(u_{j}^{\varepsilon}\right)(x)
$$

where $\varepsilon$ is a small parameter and $H\left(u_{j}^{\varepsilon}\right)(x)$ is for instance

or

$$
H\left(u_{j}^{\varepsilon}\right)(x)=\int_{\mathcal{B}_{1}(x)} u_{j}^{\varepsilon}(y) \mathrm{d} y
$$

$$
H\left(u_{j}^{\varepsilon}\right)(x)=\sup _{y \in \mathcal{B}_{1}(x)} u_{j}^{\varepsilon}(y) .
$$

Here the set $\mathcal{B}_{1}(x)$ is the unit ball centered at $x$ with respect to a smooth, uniformly convex norm $\rho$ of $\mathbb{R}^{n}$. Heuristically, this will force the populations to stay at $\rho$-distance 1 , one from each other, as $\varepsilon \rightarrow 0$.
\end{abstract}

\section{INTRODUCTION}

Segregation phenomena occur in many areas of mathematics and science: from equipartition problems in geometry, to social and biological processes (cells, bacteria, ants, mammals), to finance (sellers and buyers). There is a large body of literature in connection to our work and we would like to refer to $4,5,8,21,26,29,31,33$ and the references therein. We particularly

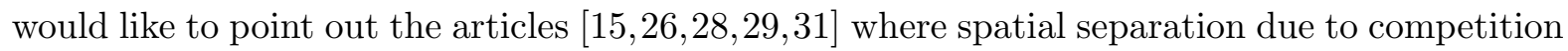
for resources is discussed among ant nests, mussels and sessile animals.

They study a family of models arising from different applications whose main two ingredients are: in the absence of competition species follow a "propagation" equation involving diffusion,

Date: July 31, 2021.

1991 Mathematics Subject Classification. Primary: 35J60; Secondary: 35R35, 35B65, 35Q92.

Key words and phrases. Regularity for viscosity solutions, Segregation of populations.

S. Patrizi was supported by the ERC grant 277749 "EPSILON Elliptic Pde's and Symmetry of Interfaces and Layers for Odd Nonlinearities". 
transport, birth-death, etc, but when two species overlap, their growth is mutually inhibited by competition, consumption of resources, etc. The simplest form of such models consists, for species $\sigma_{i}$ with spatial density $u_{i}$, on a system of equations

$$
L\left(u_{i}\right)=u_{i} F_{i}\left(u_{1}, \ldots, u_{K}\right)
$$

The operator $L$ quantifies diffusion, transport, etc, while the term $u_{i} F_{i}$ does attrition of $u_{i}$ from competition with the remaining species.

In these models, the interaction is punctual, i.e. $u_{i}(x)$ interacts with the remaining densities also at position $x$. There are many processes, though where the growth of $\sigma_{i}$ at $x$ is inhibited by the populations $\sigma_{j}$ in a full area surrounding $x$.

The purpose of this work is a first attempt to study the properties of such a segregation process. Basically, we consider a family of equations,

$$
L\left(u_{i}\right)(x)=u_{i}(x) F_{i}\left(u_{1}, \ldots, u_{K}\right)(x)
$$

where $F_{i}\left(u_{1}, \ldots, u_{K}\right)(x)$ is now a non-local factor that takes into consideration the values of $u_{j}$ in a full neighborhood of $x$. Given the previous discussion a possible model problem would be the system

$$
\Delta u_{i}^{\varepsilon}(x)=\frac{1}{\varepsilon^{2}} u_{i}^{\varepsilon}(x) \sum_{i \neq j} H\left(u_{j}^{\varepsilon}\right)(x), \quad i=1, \ldots, K
$$

where $\varepsilon$ is a small parameter and $H\left(u_{j}^{\varepsilon}\right)(x)$ is a non-local operator, for instance

$$
H\left(u_{j}^{\varepsilon}\right)(x)=\int_{B_{1}(x)} u_{j}^{\varepsilon}(y) \mathrm{d} y
$$

or

$$
H\left(u_{j}^{\varepsilon}\right)(x)=\sup _{y \in B_{1}(x)} u_{j}^{\varepsilon}(y)
$$

To study the limit configuration when the competition for resources is very high, we consider the limit when $\varepsilon$ tends to 0 . Heuristically, the non-local term forces the populations to stay at distance 1, one from each other. As an example, as we will prove, in the case of two populations in dimension two, we will have strips of length precisely one between the regions 
where the populations live. At "edge" points, that we will define as singular points, the angles of the asymptotic cones have to be the same, see Figure 1. Here $S_{i}=S_{i}^{1} \cup S_{i}^{2}, i=1,2$, represents the region where the the population $\sigma_{i}$ with density $u_{i}$ exists. Moreover, the ratio between the normal derivatives at regular points across the free boundary, depends on the ratio of the respective curvature $\varkappa$. For example, if $Z_{1} \in \partial S_{1}^{1}$ and $Z_{2} \in \partial S_{2}^{1}, Z_{1}$ and $Z_{2}$ are not "edge" points, and $d\left(Z_{1}, Z_{2}\right)=1$ then

$$
\frac{u_{\nu}^{1}\left(Z_{1}\right)}{u_{\nu}^{2}\left(Z_{2}\right)}=\frac{\varkappa\left(Z_{1}\right)}{\varkappa\left(Z_{2}\right)} \quad \text { if } \varkappa\left(Z_{2}\right) \neq 0, \quad \text { and } \quad u_{\nu}^{1}\left(Z_{1}\right)=u_{\nu}^{2}\left(Z_{2}\right) \quad \text { if } \varkappa\left(Z_{2}\right)=0 \text {. }
$$

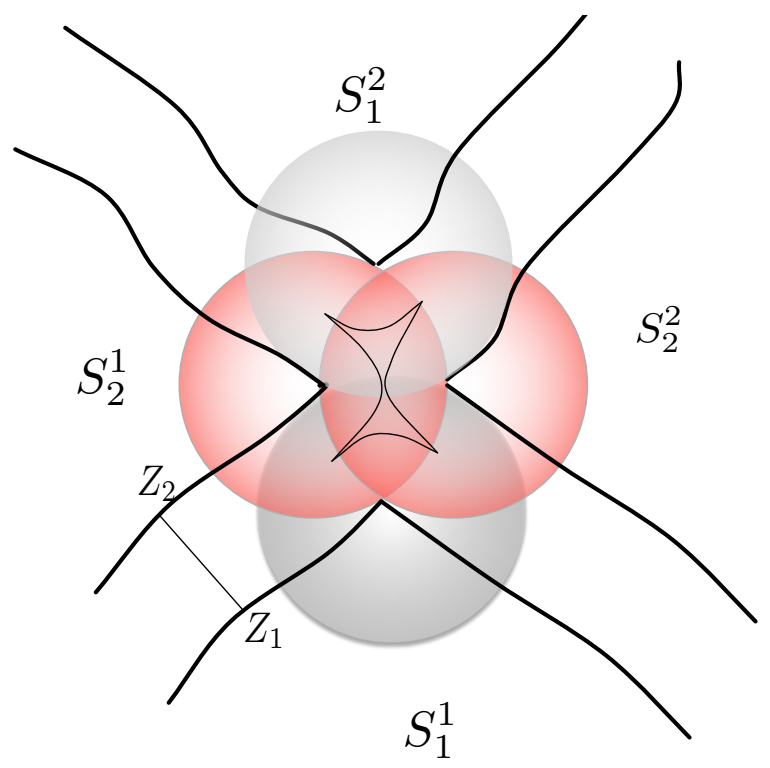

Figure 1. Example of a limit configuration for $K=2, n=2$

We will consider instead of the unit ball in the Euclidean norm $B_{1}(x)$, the translation at $x$ of a general smooth set $\mathcal{B}$ that is also uniformly convex, bounded and symmetric with respect to the origin. The set $\mathcal{B}$ defines a smooth, uniformly convex norm $\rho$ in $\mathbb{R}^{n}$.

Let us note that there is some similarity also with the Lasry-Lions model of price formation (see [6, 25]) where selling and buying prices are separated by a gap due to transaction cost. 


\section{Notation And Statement of the PRoblem}

Let $\mathcal{B}$ be an open bounded domain of $\mathbb{R}^{n}$, convex, symmetric with respect to the origin and with smooth boundary. Then $\mathcal{B}$ can be represented as the unit ball of a norm $\rho: \mathbb{R}^{n} \rightarrow \mathbb{R}$, $\rho \in C^{\infty}\left(\mathbb{R}^{n} \backslash\{0\}\right)$, called the defining function of $\mathcal{B}$, i.e.,

$$
\mathcal{B}=\left\{x \in \mathbb{R}^{n} \mid \rho(x)<1\right\}
$$

We assume that $\mathcal{B}$ is uniformly convex, i.e., there exists $0<a \leq A$ such that in $\mathbb{R}^{n} \backslash\{0\}$

$$
a I_{n} \leq D^{2}\left(\frac{1}{2} \rho^{2}\right) \leq A I_{n}
$$

where $I_{n}$ is the $n \times n$ identity matrix. In what follows we denote

$$
\begin{gathered}
\mathcal{B}_{r}:=\left\{y \in \mathbb{R}^{n} \mid \rho(y)<r\right\}, \\
\mathcal{B}_{r}(x):=\left\{y \in \mathbb{R}^{n} \mid \rho(x-y)<r\right\} .
\end{gathered}
$$

So through the paper we will always refer to the Euclidean ball as $B$ and to the $\rho$-ball as $\mathcal{B}$. For a given closed set $K$, let

$$
d_{\rho}(\cdot, K)=\inf _{y \in K} \rho(\cdot-y)
$$

be the distance function from $K$ associated to $\rho$. Then there exist $c_{1}, c_{2}>0$ such that

$$
c_{1} d(\cdot, K) \leq d_{\rho}(\cdot, K) \leq c_{2} d(\cdot, K)
$$

where $d(\cdot, K)$ is the distance function associated to the Euclidian norm $|\cdot|$ of $\mathbb{R}^{n}$.

Let $\Omega \subset \mathbb{R}^{n}$ be a bounded Lipschitz domain. We will denote by $(\partial \Omega)_{1}$ the $\rho$-strip of size 1 around $\partial \Omega$ in the complement of $\Omega$ defined by

$$
(\partial \Omega)_{1}:=\left\{x \in \Omega^{c}: d_{\rho}(x, \partial \Omega) \leq 1\right\}
$$

For $i=1, \ldots, K$, let $f_{i}$ be non-negative functions defined on $(\partial \Omega)_{1}$ with supports at $\rho$-distance equal or greater than 1, one from each other:

$$
d_{\rho}\left(\operatorname{supp} f_{i}, \operatorname{supp} f_{j}\right) \geq 1, \quad \text { for } i \neq j
$$


We will consider the following system of equations: for $i=1, \ldots, K$

$$
\begin{cases}\Delta u_{i}^{\varepsilon}(x)=\frac{1}{\varepsilon^{2}} u_{i}^{\varepsilon}(x) \sum_{j \neq i} H\left(u_{j}^{\varepsilon}\right)(x) & \text { in } \Omega, \\ u_{i}^{\varepsilon}=f_{i} & \text { on }(\partial \Omega)_{1} .\end{cases}
$$

The functional $H\left(u_{j}\right)(x)$ depends only on the restriction of $u_{j}$ to $\mathcal{B}_{1}(x)$.

We will consider, for simplicity,

$$
H(w)(x)=\int_{\mathcal{B}_{1}(x)} w^{p}(y) \varphi(\rho(x-y)) \mathrm{d} y, \quad 1 \leq p<\infty
$$

Or

$$
H(w)(x)=\sup _{\mathcal{B}_{1}(x)} w
$$

with $\varphi$ a strictly positive smooth function of $\rho$, with at most polynomial decay at $\partial \mathcal{B}_{1}$ :

$$
\varphi(\rho) \geq C(1-\rho)^{q}, \quad q \geq 0
$$

In rest of the paper, when we refer to consider $u_{1}^{\varepsilon}, \ldots, u_{K}^{\varepsilon}$, viscosity solutions of the problem (2.4), we mean that $u_{1}^{\varepsilon}, \ldots, u_{K}^{\varepsilon}$ are continuous functions that satisfy in the viscosity sense the system of equations (2.4). Moreover, we make the following assumptions: for $i=1, \ldots, K$,

$$
\left\{\begin{array}{l}
\varepsilon>0, \Omega \text { is a bounded Lipschitz domain of } \mathbb{R}^{n}, \\
f_{i}:(\partial \Omega)_{1} \rightarrow \mathbb{R}, f_{i} \geq 0, f_{i} \not \equiv 0, f_{i} \text { is Hölder continuous, } \\
\exists c>0 \text { s. t. } \forall x \in \partial \Omega \cap \operatorname{supp} f_{i},\left|\mathcal{B}_{r}(x) \cap \operatorname{supp} f_{i}\right| \geq c\left|\mathcal{B}_{r}(x)\right|, \\
(2.3 \text { holds true, } \\
H \text { is either of the form (2.5) or 2.6) and 2.7) holds. }
\end{array}\right.
$$

\section{MAin RESUlts}

For the reader's convenience we present our main results below. Assume that 2.8 holds true, then:

\section{Existence (Theorem 4.1):}

There exist continuous functions $u_{1}^{\varepsilon}, \ldots, u_{K}^{\varepsilon}$, depending on the parameter $\varepsilon$, viscosity solutions of the problem (2.4). 


\section{Limit problem (Corollary 5.6):}

There exists a subsequence $(\vec{u})^{\varepsilon_{m}}$ converging locally uniformly, as $\varepsilon \rightarrow 0$, to a function $\vec{u}=\left(u_{1}, \ldots, u_{K}\right)$, satisfying the following properties:

i) the $u_{i}$ 's are locally Lipschitz continuous in $\Omega$ and have supports at distance at least 1, one from each other, i.e.

$$
u_{i} \equiv 0 \quad \text { in the set } \quad\left\{x \in \Omega \mid d_{\rho}\left(x, \text { supp } u_{j}\right) \leq 1\right\} \quad \text { for any } j \neq i .
$$

ii) $\Delta u_{i}=0$ when $u_{i}>0$.

\section{Semiconvexity of the free boundary (Corollary 6.2):}

If $x_{0} \in \partial\left\{u_{i}>0\right\}$ there is an exterior tangent $\rho$-ball of radius 1 at $x_{0}$.

The supports of $u_{i}$ are sets of finite perimeter (Corollary 6.5):

The set $\left\{u_{i}>0\right\}$ has finite perimeter.

\section{Sharp characterization of the interfaces (Theorem 7.1):}

Under the additional assumption that $p=1$ in (2.5), the supports of the limit functions are at distance exactly 1 , one from each other, i.e, if $x_{0} \in \partial\left\{u_{i}>0\right\} \cap \Omega$, then there exists $j \neq i$ such that

$$
\overline{\mathcal{B}_{1}\left(x_{0}\right)} \cap \partial\left\{u_{j}>0\right\} \neq \emptyset
$$

\section{Classification of singular points in dimension 2 (Lemma 8.9 , Theorem 8.10 ,} Corollary 8.11, Corollary 8.12):

For $n=2$, under the additional assumption that $p=1$ in 2.5), for $i \neq j$, let $x_{0} \in$ $\partial\left\{u_{i}>0\right\} \cap \Omega$ and $y_{0} \in \partial\left\{u_{j}>0\right\} \cap \Omega$ be points such that $\left\{u_{i}>0\right\}$ has an angle $\theta_{i}$ at $x_{0},\left\{u_{j}>0\right\}$ has an angle $\theta_{j}$ at $y_{0}$ and $\rho\left(x_{0}-y_{0}\right)=1$. Then we have

$$
\begin{gathered}
\theta_{i}=\theta_{j} . \\
\text { If } x_{0} \in \partial\left\{u_{i}>0\right\} \cap \partial \Omega \text { and } y_{0} \in \partial\left\{u_{j}>0\right\} \cap \Omega, \text { then } \\
\theta_{i} \leq \theta_{j} .
\end{gathered}
$$


Moreover, singular points, i.e. points where the free boundaries have corners, are isolated and finite. If the domain is a strip and there are only two populations, under additional monotonicity assumptions on the boundary data, the free boundary sets $\partial\left\{u_{i}>0\right\}$, $i=1,2$, are of class $C^{1}$.

\section{Lipschitz regularity for free boundary for the obstacle problem associated in dimension 2 (Theorem 8.18):}

For $n=2$, under the additional assumption that $p=1$ in (2.5), $f_{i} \equiv 1$ and additional conditions about the regularity of $\partial \Omega$, if $\left(u_{1}^{\varepsilon}, \ldots, u_{K}^{\varepsilon}\right)$ is a particular solution of 2.4 which satisfies the associated obstacle problem 8.49 with $\left(u_{1}, \ldots, u_{K}\right)$ the limit as $\varepsilon \rightarrow$ 0 , then the free boundaries $\partial\left\{u_{i}>0\right\}, i=1, \ldots, K$, are Lipschitz curves of the plane.

\section{Free boundary condition (Theorem 9.2):}

In any dimension, assume that we have 2 populations, $H$ is defined as in (2.5) with $\varphi \equiv 1, p=1$ and $\mathcal{B}_{1}(x)=B_{1}(x)$ is the Euclidian ball, $0 \in \partial\left\{u_{1}>0\right\}, e_{n} \in \partial\left\{u_{2}>0\right\}$, and $\partial\left\{u_{1}>0\right\}$ and $\partial\left\{u_{2}>0\right\}$ are of class $C^{2}$ in a neighborhood of 0 and $e_{n}$ respectively. Let $\varkappa_{i}(0)$ denote the principal curvatures of $\partial\left\{u_{1}>0\right\}$ at 0 , where outward is the positive direction and let $\varkappa_{i}\left(e_{n}\right)$ denote the principal curvatures of $\partial\left\{u_{2}>0\right\}$ at $e_{n}$ where now inward is the positive direction. Then, we have the following relation on the exterior normal derivatives of $u_{1}$ and $u_{2}$ :

$$
\frac{u_{\nu}^{1}(0)}{u_{\nu}^{2}\left(e_{n}\right)}=\prod_{\substack{i=1 \\ \varkappa_{i}(0) \neq 0}}^{n-1} \frac{\varkappa_{i}(0)}{\varkappa_{i}\left(e_{n}\right)} \quad \text { if } \varkappa_{i}(0) \neq 0 \text { for some } i=1, \ldots, n-1
$$

and

$$
u_{\nu}^{1}(0)=u_{\nu}^{2}\left(e_{n}\right) \quad \text { if } \varkappa_{i}(0)=0 \text { for any } i=1, \ldots, n-1 \text {. }
$$




\section{Existence OF SOLUTiOns}

This proof follows the same steps as in 30 and it is written below for the reader's convenience.

Theorem 4.1. Assume 2.8]. Then there exist continuous positive functions $u_{1}^{\varepsilon}, \ldots, u_{K}^{\varepsilon}$, depending on the parameter $\varepsilon$, viscosity solutions of the problem 2.4.

Proof. The proof uses a fixed point result. Let $B$ be the Banach space of bounded continuous vector-valued functions defined on the domain $\Omega$ with the norm

$$
\left\|\left(u_{1}, u_{2}, \ldots, u_{K}\right)\right\|_{B}:=\max _{i}\left(\sup _{x \in \Omega}\left|u_{i}(x)\right|\right) .
$$

For $i=1, \ldots, K$, let $\phi_{i}$ be the solutions of

$$
\begin{cases}\Delta \phi_{i}=0 & \text { in } \Omega, \\ \phi_{i}=f_{i} & \text { on } \partial \Omega .\end{cases}
$$

Let $\Theta$ be the subset of bounded continuous functions in $\Omega$, that satisfy prescribed boundary data, and are bounded from above and from below as stated below:

$$
\Theta=\left\{\left(u_{1}, u_{2}, \ldots, u_{K}\right) \mid u_{i}: \Omega \rightarrow \mathbb{R} \text { is continuous, } 0 \leq u_{i} \leq \phi_{i} \text { in } \Omega, u_{i}=f_{i} \text { on }(\partial \Omega)_{1}\right\}
$$

Notice that $\Theta$ is a closed and convex subset of $B$. Let $T^{\varepsilon}$ be the operator that is defined on $\Theta$ in the following way: $T^{\varepsilon}\left(\left(u_{1}, u_{2}, \ldots, u_{K}\right)\right):=\left(v_{1}^{\varepsilon}, v_{2}^{\varepsilon}, \ldots, v_{K}^{\varepsilon}\right)$ if for any $i=1, \ldots, K, v_{i}^{\varepsilon}$ is solution to the following problem:

$$
\begin{cases}\Delta\left(v_{i}^{\varepsilon}\right)(x)=\frac{1}{\varepsilon^{2}} v_{i}^{\varepsilon}(x) \sum_{j \neq i} H\left(u_{j}\right)(x) & \text { in } \Omega \\ v_{i}^{\varepsilon}=f_{i} & \text { on }(\partial \Omega)_{1},\end{cases}
$$

where $u_{j}, j \neq i$ are given. Observe that if $T^{\varepsilon}$ has a fixed point

$$
T^{\varepsilon}\left(\left(u_{1}^{\varepsilon}, u_{2}^{\varepsilon}, \ldots, u_{K}^{\varepsilon}\right)\right)=\left(u_{1}^{\varepsilon}, u_{2}^{\varepsilon}, \ldots, u_{K}^{\varepsilon}\right)
$$

then $\left(u_{1}^{\varepsilon}, u_{2}^{\varepsilon}, \ldots, u_{K}^{\varepsilon}\right)$ is a solution of problem 2.4 .

In order for $T^{\varepsilon}$ to have a fixed point, we need to prove that it satisfies the hypothesis of the Schauder fixed point Theorem, see 23:

(1) $T^{\varepsilon}(\Theta) \subset \Theta$ : 
Classical existence results guarantee the existence of a viscosity solution $\left(v_{1}^{\varepsilon}, v_{2}^{\varepsilon}, \ldots, v_{K}^{\varepsilon}\right)$ of problem 4.2 which is smooth in $\Omega$. Since $f_{i} \geq 0$ and $f_{i} \not \equiv 0$, the strong maximum principle implies

$$
v_{i}^{\varepsilon}>0 \quad \text { in } \Omega \text {. }
$$

This implies that

$$
\Delta v_{i}^{\varepsilon} \geq 0 \quad \text { in } \Omega
$$

and, again from the comparison principle, we have

$$
v_{i}^{\varepsilon} \leq \phi_{i} \quad \text { in } \Omega
$$

We have proved that $T^{\varepsilon}\left(\left(u_{1}, u_{2}, \ldots, u_{K}\right)\right) \in \Theta$.

(2) $T^{\varepsilon}$ is continuous:

Let us assume that $\left(\left(u_{1}\right)_{m}, \ldots,\left(u_{K}\right)_{m}\right) \rightarrow\left(u_{1}, \ldots, u_{K}\right)$ in $B$ meaning that when $m$ tends to $+\infty$,

$$
\max _{1 \leq i \leq K}\left\|\left(u_{i}\right)_{m}-u_{i}\right\|_{L^{\infty}} \rightarrow 0
$$

We need to prove that for each fixed $\varepsilon>0$

$$
\left\|T^{\varepsilon}\left(\left(u_{1}\right)_{m}, \ldots,\left(u_{K}\right)_{m}\right)-T^{\varepsilon}\left(u_{1}, \ldots, u_{K}\right)\right\|_{B} \rightarrow 0
$$

when $m \rightarrow+\infty$. Let

$$
T^{\varepsilon}\left(\left(u_{1}\right)_{m}, \ldots,\left(u_{K}\right)_{m}\right)=\left(\left(v_{1}^{\varepsilon}\right)_{m}, \ldots,\left(v_{K}^{\varepsilon}\right)_{m}\right)
$$

then if we prove that there exists a constant $C_{\varepsilon}$ independent of $m$, so that we have the estimate, for $i=1, \ldots, K$

$$
\left\|\left(v_{i}^{\varepsilon}\right)_{m}-v_{i}^{\varepsilon}\right\|_{L^{\infty}} \leq C_{\varepsilon} \max _{j}\left\|\left(u_{j}\right)_{m}-u_{j}\right\|_{L^{\infty}}
$$

the result follows. For all $x \in \Omega$ and for fixed $i$, let $\omega_{m}$ be the function

$$
\omega_{m}(x)=\left(v_{i}^{\varepsilon}\right)_{m}(x)-v_{i}^{\varepsilon}(x),
$$


and suppose for instance that there exists $y \in \Omega$ such that

$$
\omega_{m}(y)>r^{2} D \max _{j}\left\|\left(u_{j}\right)_{m}-u_{j}\right\|_{L^{\infty}}
$$

for some large $D>0$, where $r$ is such that $\Omega \subset B_{r}$, and $B_{r}$ is the ball centered at 0 of radius $r$ in the Euclidean norm. We want to prove that this is impossible if $D$ is sufficiently large. Let $h_{m}$ be the concave radially symmetric function

$$
h_{m}(x)=\gamma_{m}\left(r^{2}-|x|^{2}\right)
$$

with $\gamma_{m}=D \max _{j}\left\|\left(u_{j}\right)_{m}-u_{j}\right\|_{L^{\infty}}$. Observe that:

(a) $h_{m}(x)=0$ on $\partial B_{r}$;

(b) $h_{m}(x) \leq r^{2} D \max _{j}\left\|\left(u_{j}\right)_{m}-u_{j}\right\|_{L^{\infty}}$ for all $x$ in $B_{r}$;

(c) $0=\omega_{m}(x) \leq h_{m}(x)$ on $\partial \Omega$, since $\left(v_{i}^{\varepsilon}\right)_{m}$ and $v_{i}^{\varepsilon}$ are solutions with the same boundary data.

Since we are assuming (4.4), there exists a negative minimum of $h_{m}-\omega_{m}$ in $\Omega$. Let $x_{0} \in \Omega$ be a point where the minimum value of $h_{m}-\omega_{m}$ is attained. Then

$$
h_{m}\left(x_{0}\right)-\omega_{m}\left(x_{0}\right)<0 \quad \text { and } \quad \Delta\left(h_{m}-\omega_{m}\right)\left(x_{0}\right) \geq 0 .
$$

Then, we have

$$
\begin{aligned}
\Delta \omega_{m}\left(x_{0}\right) & =\Delta\left(\left(v_{i}^{\varepsilon}\right)_{m}\right)\left(x_{0}\right)-\Delta v_{i}^{\varepsilon}\left(x_{0}\right) \\
& =\frac{1}{\varepsilon^{2}}\left(\left(\left(v_{i}^{\varepsilon}\right)_{m}\left(x_{0}\right)-v_{i}^{\varepsilon}\left(x_{0}\right)\right) \sum_{j \neq i} H\left(\left(u_{j}\right)_{m}\right)\left(x_{0}\right)\right. \\
& \left.-v_{i}^{\varepsilon}\left(x_{0}\right) \sum_{j \neq i}\left(H\left(u_{j}\right)\left(x_{0}\right)-H\left(\left(u_{j}\right)_{m}\right)\left(x_{0}\right)\right)\right) \\
& \geq \frac{1}{\varepsilon^{2}}\left(\left(\left(v_{i}^{\varepsilon}\right)_{m}\left(x_{0}\right)-v_{i}^{\varepsilon}\left(x_{0}\right)\right) \sum_{j \neq i} H\left(\left(u_{j}\right)_{m}\right)\left(x_{0}\right)\right. \\
& \left.-v_{i}^{\varepsilon}\left(x_{0}\right)(K-1) C \max _{j}\left\|\left(u_{j}\right)_{m}-u_{j}\right\|_{L^{\infty}(\Omega)}\right)
\end{aligned}
$$


adding and subtracting $\frac{1}{\varepsilon^{2}} v_{i}^{\varepsilon}\left(x_{0}\right) \sum_{j \neq i} H\left(\left(u_{j}\right)_{m}\right)\left(x_{0}\right)$, where $C$ depends on the $f_{j}$ 's and $\varphi$. Then

$$
\begin{aligned}
0 & \leq \Delta\left(h_{m}-\omega_{m}\right)\left(x_{0}\right) \\
& \leq-2 \gamma_{m} n-\frac{1}{\varepsilon^{2}}\left(\left(\left(v_{i}^{\varepsilon}\right)_{m}-v_{i}^{\varepsilon}\right)\left(x_{0}\right) \sum_{j \neq i} H\left(\left(u_{j}\right)_{m}\right)\left(x_{0}\right)\right. \\
& \left.-v_{i}^{\varepsilon}\left(x_{0}\right)(K-1) C \max _{j}\left\|\left(u_{j}\right)_{m}-u_{j}\right\|_{L^{\infty}}\right) \\
& \leq-2 n D \max _{j}\left\|\left(u_{j}\right)_{m}-u_{j}\right\|_{L^{\infty}}+\frac{1}{\varepsilon^{2}} v_{i}^{\varepsilon}\left(x_{0}\right)(K-1) C \max _{j}\left\|\left(u_{j}\right)_{m}-u_{j}\right\|_{L^{\infty}} \\
& \leq-2 n D \max _{j}\left\|\left(u_{j}\right)_{m}-u_{j}\right\|_{L^{\infty}}+\frac{\widetilde{C}}{\varepsilon^{2}} \max _{j}\left\|\left(u_{j}\right)_{m}-u_{j}\right\|_{L^{\infty}}
\end{aligned}
$$

because $0<h_{m}\left(x_{0}\right)<\omega_{m}\left(x_{0}\right)=\left(\left(v_{i}^{\varepsilon}\right)_{m}-v_{i}^{\varepsilon}\right)\left(x_{0}\right)$ and $\sum_{j \neq i} H\left(\left(u_{j}\right)_{m}\right)\left(x_{0}\right) \geq 0$ and so

$$
-\frac{1}{\varepsilon^{2}}\left(\left(v_{i}^{\varepsilon}\right)_{m}-v_{i}^{\varepsilon}\right)\left(x_{0}\right) \sum_{j \neq i} H\left(\left(u_{j}\right)_{m}\right)\left(x_{0}\right) \leq 0 .
$$

Taking $D=D_{\varepsilon}>\frac{\widetilde{C}}{2 n \varepsilon^{2}}$, we obtain that

$$
0 \leq \Delta\left(h_{m}-\omega_{m}\right)\left(x_{0}\right)<0
$$

which is a contradiction.

(3) $T^{\varepsilon}(\Theta)$ is precompact:

Let $\left(\left(u_{1}\right)_{m}, \ldots,\left(u_{K}\right)_{m}\right)$ be a bounded sequence in $B$ and let

$$
\left(\left(v_{1}^{\varepsilon}\right)_{m}, \ldots,\left(v_{K}^{\varepsilon}\right)_{m}\right)=T^{\varepsilon}\left(\left(u_{1}\right)_{m}, \ldots,\left(u_{K}\right)_{m}\right)
$$

Then by standard Hölder estimates for viscosity solutions, $\left(\left(v_{1}^{\varepsilon}\right)_{m}, \ldots,\left(v_{K}^{\varepsilon}\right)_{m}\right)$ is bounded in the space of Hölder continuous functions on $\bar{\Omega}$. Since the subset of $\Theta$ of Hölder continuous functions on $\bar{\Omega}$ is precompact in $\Theta$, we can extract from $\left(\left(v_{1}^{\varepsilon}\right)_{m}, \ldots,\left(v_{K}^{\varepsilon}\right)_{m}\right)$ a subsequence which is converging in $B$.

We have proven the existence of a solution $\left(u_{1}^{\varepsilon}, \ldots, u_{K}^{\varepsilon}\right)$ of 2.4 . The same argument as in (1) shows that $u_{i}^{\varepsilon}>0$ in $\Omega$. This concludes the proof of the theorem. 


\section{UNIFORM IN $\varepsilon$ LIPSCHITZ ESTIMATES}

In this section we will prove uniform in $\varepsilon$ Lipschitz estimates that will imply the convergence, up to subsequences, of the solution $\left(u_{1}^{\varepsilon}, \ldots, u_{K}^{\varepsilon}\right)$ of 2.4 to a limit function $\left(u_{1}, \ldots, u_{K}\right)$ as $\varepsilon \rightarrow 0$. We will show that the functions $u_{i}$ 's are locally Lipschitz continuous in $\Omega$ and harmonic inside their support. Moreover, $u_{i} \equiv 0$ in the $\rho$-strip of size 1 of the support of $u_{j}$ for any $j \neq i$, i.e., the supports of the limit functions are at distance at least 1, one from each other. We start by proving general properties of subsolutions of uniform elliptic equations.

\section{Lemma 5.1. Let:}

a) $\omega$ be a subharmonic function in $\mathcal{B}_{1}$, such that

a) $\omega \leq 1$ in $\mathcal{B}_{1}$

$\left.\mathrm{a}_{2}\right) \omega(0)=m>0$.

b) $D_{0}$ be a smooth convex set with bounded curvatures

$$
\left|\varkappa_{i}\left(\partial D_{0}\right)\right| \leq C_{0}, \quad i=1, \ldots, n-1
$$

(like $\mathcal{B}_{1}$ above).

Then, there exists a universal $\tau_{0}=\tau_{0}\left(C_{0}, n, \rho\right)$ such that, if the distance $d_{\rho}\left(D_{0}, 0\right) \leq \tau_{0} m$, then

$$
\sup _{\partial D_{0} \cap \mathcal{B}_{1}} \omega \geq \frac{m}{2}
$$

Proof. Assume w.l.o.g. that $0 \notin D_{0}$ and let $h$ be harmonic in $\mathcal{B}_{1} \backslash D_{0}$ and such that

$$
\left\{\begin{array}{l}
h=1 \text { on }\left(\partial \mathcal{B}_{1}\right) \backslash D_{0} \\
h=\frac{m}{2} \text { on }\left(\partial D_{0}\right) \cap \mathcal{B}_{1} .
\end{array}\right.
$$

By assumption (b), the set $\mathcal{B}_{1} \backslash D_{0}$ satisfies an exterior uniform ball condition at any point of $\partial D_{0} \cap \mathcal{B}_{1}$, therefore, by a standard barrier argument, $h$ grows no more than linearly away from $\partial D_{0}$ in $\mathcal{B}_{\frac{1}{2}}$, i.e., there exist $k_{1}, k_{2}>0$ depending on $C_{0}$ and $n$ such that, if $x \in \mathcal{B}_{\frac{1}{2}} \backslash D_{0}$ and $d\left(x, \partial D_{0}\right) \leq k_{2}$, then $h(x) \leq k_{1} d\left(x, \partial D_{0}\right)+\frac{m}{2}$. To prove that $h(0)<m$ observe that if $\tau_{0} \leq k_{2} c_{1}$, where $c_{1}$ is given by $(2.2)$, then $d\left(0, \partial D_{0}\right) \leq \tau_{0} m / c_{1} \leq k_{2} m \leq k_{2}$ and therefore, if in 
addition $\tau_{0}$ is so small that $\frac{k_{1}}{c_{1}} \tau_{0} \leq \frac{1}{2}$, we have

$$
h(0) \leq k_{1} d\left(0, \partial D_{0}\right)+\frac{m}{2} \leq \frac{k_{1}}{c_{1}} d_{\rho}\left(0, \partial D_{0}\right)+\frac{m}{2} \leq \frac{k_{1}}{c_{1}} \tau_{0} m+\frac{m}{2}<m .
$$

Hence, we must have $\sup _{\left(\partial D_{0} \cap \mathcal{B}_{1}\right)} \omega \geq \frac{m}{2}$, otherwise the comparison principle would imply $\omega(x) \leq h(x)$ in $\mathcal{B}_{1} \backslash D_{0}$, which is a contradiction at $x=0$.

Lemma 5.2. Let $\omega$ be a positive subsolution of a uniformly elliptic equation, $\left(\lambda^{2} I \leq a_{i j} \leq \Lambda^{2} I\right)$

$$
a_{i j} D_{i j} \omega \geq \theta^{2} \omega \quad \text { in } \mathcal{B}_{r}
$$

Then there exist $c, C>0$ such that

$$
\frac{\omega(0)}{\sup _{\mathcal{B}_{r}} \omega} \leq C \mathrm{e}^{-c \theta r}
$$

Proof. The function

$$
g(x)=\sum_{i=1}^{n} \cosh \left(\frac{\theta}{\Lambda} x_{i}\right)
$$

is a supersolution of the equation $a_{i j} D_{i j} u=\theta^{2} u$. Moreover, using the convexity of the exponential function, it is easy to check that it satisfies

$$
g(x) \geq C_{1} e^{c \theta r} \quad \text { for any } x \in \partial \mathcal{B}_{r} .
$$

Then, the comparison principle implies

$$
\frac{\omega(x)}{\sup _{\mathcal{B}_{r}} \omega} \leq \frac{g(x)}{C_{1} e^{c \theta r}} \quad \text { for any } x \in \mathcal{B}_{r} .
$$

The result follows taking $x=0$.

The next lemma says that if $u_{i}^{\varepsilon}$ attains a positive value $\sigma$ at some interior point, then all the other functions $u_{j}^{\varepsilon}, j \neq i$, go to zero exponentially in a $\rho$-ball of radius $1+c \sigma$ around that point.

Lemma 5.3. Assume 2.8). Let $\left(u_{1}^{\varepsilon}, \ldots, u_{K}^{\varepsilon}\right)$ be a viscosity solution of the problem (2.4). For $i=1, \ldots, K, \sigma>0$, and $0<r<1$ let

$$
\Gamma_{i}^{\sigma, r}:=\left\{y \in \Omega: d_{\rho}\left(y, \operatorname{supp} f_{i}\right) \geq 2 r, u_{i}^{\varepsilon}=\sigma\right\}
$$


and

$$
m:=\frac{\sigma}{\sup _{\partial \Omega} f_{i}}
$$

Then, there exists a universal constant $0<\tau<1$ such that, in the sets

$$
\Sigma_{i, j}^{\sigma, r}:=\left\{x \in \Omega: d_{\rho}\left(x, \Gamma_{i}^{\sigma, r}\right) \leq 1+\frac{\tau m r}{2}, d_{\rho}\left(x, \operatorname{supp} f_{j}\right) \geq \frac{\tau m r}{4}\right\}
$$

we have

$$
u_{j}^{\varepsilon} \leq C e^{-\frac{c \sigma^{\alpha} r^{\beta}}{\varepsilon}}, \quad \text { for } j \neq i,
$$

for some positive $\alpha$ and $\beta$ depending on the structure of $H$ ( $p$ and $q)$.

Proof. Let $0<\tau<1$ to be determined. For $0<r<1$, let us consider the set $\Sigma_{i, j}^{\sigma, r}$ defined above and let $\bar{x} \in \Sigma_{i, j}^{\sigma, r}$. We want to show that for $j \neq i$, we have

$$
\Delta u_{j}^{\varepsilon} \geq \frac{C \sigma^{\bar{\alpha}} r^{\bar{\beta}}}{\varepsilon^{2}} u_{j}^{\varepsilon} \quad \text { in } \mathcal{B}_{\frac{\tau m r}{4}}(\bar{x})
$$

for some $\bar{\alpha}, \bar{\beta}>0$. Let us prove it for $\bar{x}$ such that $d_{\rho}\left(\bar{x}, \Gamma_{i}^{\sigma, r}\right)=1+\frac{\tau m r}{2}$, which is the hardest case. First of all, remark that since $d_{\rho}\left(\bar{x}, \operatorname{supp} f_{j}\right) \geq \frac{\tau m r}{4}$, the ball $\mathcal{B}_{\frac{\tau m r}{4}}(\bar{x})$ does not intersect $\operatorname{supp} f_{j}$. Therefore, $u_{j}^{\varepsilon}$ (which is eventually zero in $\mathcal{B}_{\frac{\tau m r}{4}}(\bar{x}) \cap \Omega^{c}$ ) satisfies

$$
\Delta u_{j}^{\varepsilon} \geq \frac{1}{\varepsilon^{2}} u_{j}^{\varepsilon} \sum_{k \neq j} H\left(u_{k}^{\varepsilon}\right) \quad \text { in } \mathcal{B}_{\frac{\tau m r}{4}}(\bar{x}) .
$$

Next, the ball $\mathcal{B}_{1-\frac{\tau m r}{2}}(\bar{x})$ is at distance $\tau m r$ from a point $y \in \Gamma_{i}^{\sigma, r}$. Remark that since $\mathcal{B}_{2 r}(y) \cap$ $\operatorname{supp} f_{i}=\emptyset$, the function $u_{i}^{\varepsilon}$ (which is eventually equal to zero in $\mathcal{B}_{2 r}(y) \cap \Omega^{c}$ ) satisfies $\Delta u_{i}^{\varepsilon} \geq 0$ in $\mathcal{B}_{2 r}(y)$. Moreover, since $u_{i}^{\varepsilon}$ is subharmonic in $\Omega$, it attains its maximum at the boundary of $\Omega$, so that $u_{i}^{\varepsilon} / \sup _{\partial \Omega} f_{i} \leq 1$ in $\Omega$. In particular $m=\frac{\sigma}{\sup _{\partial \Omega} f_{i}} \leq 1$. Set

$$
v(x):=\frac{u_{i}^{\varepsilon}(y+r x)}{\sup _{\partial \Omega} f_{i}},
$$

then $v \leq 1$ and $v(0)=u_{i}^{\varepsilon}(y) / \sup _{\partial \Omega} f_{i}=\sigma / \sup _{\partial \Omega} f_{i}=m$ and $\Delta v \geq 0$ in $\mathcal{B}_{1}$. Let

$$
D_{0}:=\mathcal{B}_{\frac{1}{r}-\frac{\tau m}{2}}\left(\frac{\bar{x}-y}{r}\right),
$$

then the principal curvatures of $D_{0}$ satisfy

$$
\left|\varkappa_{i}\left(\partial D_{0}\right)\right| \leq \frac{C_{\rho}}{\frac{1}{r}-\frac{\tau m}{2}}=\frac{2 r C_{\rho}}{2-r \tau m}<2 r C_{\rho}<2 C_{\rho} .
$$


Moreover $D_{0}$ is at distance $\tau m$ from 0. Hence, from Lemma 5.1 applied to the function $v$ given by (5.3) with $D_{0}$ defined as above, if $\tau=\min \left\{1, \tau_{0}\right\}$, where $\tau_{0}$ is the universal constant given by the lemma, then there is a point $z$ in $\partial \mathcal{B}_{1-\frac{\tau m r}{2}}(\bar{x}) \cap \mathcal{B}_{r}(y)$, such that $u_{i}^{\varepsilon}(z) \geq \sigma / 2$. Next, remark that if $x \in \mathcal{B}_{\frac{\tau m r}{4}}(\bar{x})$ then

$$
\mathcal{B}_{1}(x) \supset \mathcal{B}_{\frac{\tau m r}{4}}(z)
$$

(since $\left.d_{\rho}(x, z) \leq d_{\rho}(x, \bar{x})+d_{\rho}(\bar{x}, z) \leq \frac{\tau m r}{4}+1-\frac{\tau m r}{2}=1-\frac{\tau m r}{4}\right)$.

Let us first consider the case $H$ defined as in 2.6). Then for any $x \in \mathcal{B}_{\frac{\tau m r}{4}}(\bar{x})$ we have

$$
H\left(u_{i}^{\varepsilon}\right)(x)=\sup _{\mathcal{B}_{1}(x)} u_{i}^{\varepsilon} \geq u_{i}^{\varepsilon}(z) \geq \frac{\sigma}{2}
$$

which, with together $(5.2)$, implies 5.1$)$ with $\bar{\alpha}=1$ and $\bar{\beta}=0$.

Next, let us turn to the case $H$ defined as in 2.5). Remark that since $z \in \mathcal{B}_{r}(y)$ and $d_{\rho}\left(y\right.$, supp $\left.f_{i}\right) \geq 2 r$, we have that $\mathcal{B}_{r}(z) \cap \operatorname{supp} f_{i}=\emptyset$ and therefore the function $u_{i}^{\varepsilon}$ (which is eventually equal to zero in $\mathcal{B}_{r}(z) \cap \Omega^{c}$ ) satisfies $\Delta u_{i}^{\varepsilon} \geq 0$ in $\mathcal{B}_{r}(z)$. This implies that $\left(u_{i}^{\varepsilon}\right)^{p}$, $p \geq 1$, is subharmonic in $\mathcal{B}_{r}(z)$ and by the mean value inequality

$$
f_{B_{s}(z)}\left(u_{i}^{\varepsilon}\right)^{p} d x \geq\left(\frac{\sigma}{2}\right)^{p}
$$

in any Euclidian ball $B_{s}(z) \subset \mathcal{B}_{r}(z)$, for any $p \geq 1$. Since $d_{\rho}$ and the Euclidian distance are equivalent, there is an $s \sim \tau m r$ such that

$$
B_{s}(z) \subset \mathcal{B}_{\frac{\tau m r}{8}}(z) \subset \mathcal{B}_{\frac{\tau m r}{4}}(z) \subset \mathcal{B}_{1}(x)
$$

Moreover, if $y \in B_{s}(z)$ and $x \in \mathcal{B}_{\frac{\tau m r}{4}}(\bar{x})$, then

$$
\rho(y-x) \leq \rho(y-z)+\rho(z-\bar{x})+\rho(\bar{x}-x) \leq \frac{\tau m r}{8}+\left(1-\frac{\tau m r}{2}\right)+\frac{\tau m r}{4}=1-\frac{\tau m r}{8},
$$

that is

$$
1-\rho(y-x) \geq \frac{\tau m r}{8}
$$


Hence, using (5.5), (2.7), 5.6) and (5.4), for all $x \in \mathcal{B}_{\frac{\tau m r}{4}}(\bar{x})$ we get

$$
\begin{aligned}
H\left(u_{i}^{\varepsilon}\right)(x) & =\int_{\mathcal{B}_{1}(x)}\left(u_{i}^{\varepsilon}\right)^{p}(y) \varphi(\rho(y-x)) d y \\
& \geq \int_{B_{s}(z)}\left(u_{i}^{\varepsilon}\right)^{p}(y) C(1-\rho(y-x))^{q} d y \\
& \geq \int_{B_{s}(z)}\left(u_{i}^{\varepsilon}\right)^{p}(y) C\left(\frac{\tau m r}{8}\right)^{q} d y \\
& \geq C \sigma^{\bar{\alpha}} r^{\bar{\beta}}
\end{aligned}
$$

where $\bar{\alpha}$ and $\bar{\beta}$ depend on $p, q$ and on the dimension $n$. This and (5.2) imply (5.1).

Now, by Lemma 5.2 we get

$$
u_{j}^{\varepsilon}(\bar{x}) \leq C e^{-\frac{c \sigma^{\alpha} r^{\beta}}{\varepsilon}}
$$

for $\alpha=\frac{\bar{\alpha}}{2}+1$ and $\beta=\frac{\bar{\beta}}{2}+1$, and the lemma is proven.

Corollary 5.4. Assume (2.8). Let $\left(u_{1}^{\varepsilon}, \ldots, u_{K}^{\varepsilon}\right)$ be a viscosity solution of the problem (2.4). Let $y$ be a point in $\Omega$ such that

$$
u_{i}^{\varepsilon}(y)=\sigma, \quad d_{\rho}\left(y, \operatorname{supp} f_{j}\right) \geq 1+\tau m r, \quad i \neq j \quad \text { and } \quad d_{\rho}(y, \partial \Omega) \geq 2 r
$$

where $m=\frac{\sigma}{\sup _{\partial \Omega} f_{i}}, 0<r<1, \varepsilon \leq \sigma^{2 \alpha} r^{2 \beta}$ and $\tau, \alpha$ and $\beta$ are given by Lemma 5.3. Then there exists a constant $C_{0}>0$ such that in $\mathcal{B}_{\frac{\tau m r}{4}}(y)$ we have

$$
\left|\nabla u_{i}^{\varepsilon}\right| \leq \frac{C_{0}}{r}
$$

and

$$
\Delta u_{i}^{\varepsilon} \rightarrow 0 \text { as } \varepsilon \rightarrow 0 \text { uniformly }
$$

Proof. First of all, remark that $m \leq 1$, as $u_{i}$ attains its maximum at the boundary of $\Omega$. Since in addition $\tau<1$, we have that $\mathcal{B}_{\frac{\tau m r}{2}}(y) \subset \mathcal{B}_{2 r}(y) \subset \Omega$. Therefore, we use 2.4) to estimate $\Delta u_{i}^{\varepsilon}(z)$, for $z \in \mathcal{B}_{\frac{\tau m r}{2}}(y)$. In order to do that, we need to estimate $H\left(u_{j}^{\varepsilon}\right)(z)$ for $j \neq i$. But $H\left(u_{j}^{\varepsilon}\right)(z)$ involves points $x$ at $\rho$-distance 1 from $z$. Let $x$ be such that $d_{\rho}(x, z) \leq 1$, then 
$d_{\rho}(x, y) \leq 1+\frac{\tau m r}{2}$. Moreover, since $d_{\rho}\left(y, \operatorname{supp} f_{j}\right) \geq 1+\tau m r$, we have $d_{\rho}\left(x\right.$, supp $\left.f_{j}\right) \geq \frac{\tau m r}{2}$.

Hence, by Lemma 5.3 , for any $j \neq i$

$$
u_{j}^{\varepsilon}(x) \leq C e^{-\frac{c \sigma^{\alpha} r^{\beta}}{\varepsilon}} \quad \text { for } x \in \mathcal{B}_{1}(z)
$$

From the previous estimate and (2.4), it follows that for $z \in \mathcal{B}_{\frac{\tau m r}{2}}(y)$ we have

$$
0 \leq \Delta u_{i}^{\varepsilon}(z) \leq u_{i}^{\varepsilon}(z) \frac{C e^{-\frac{c \sigma^{\alpha} r^{\beta}}{\varepsilon}}}{\varepsilon^{2}} \leq u_{i}^{\varepsilon}(z) \frac{C e^{-c \varepsilon^{-\frac{1}{2}}}}{\varepsilon^{2}}=o(1) \quad \text { as } \varepsilon \rightarrow 0,
$$

for $\varepsilon \leq \sigma^{2 \alpha} r^{2 \beta}$. If we normalize the ball $\mathcal{B}_{\frac{\tau m r}{2}}(y)$ in a Lipschitz fashion:

$$
\bar{u}_{i}^{\varepsilon}(\bar{z}):=2 \frac{u_{i}^{\varepsilon}\left(\frac{\tau m r}{2} \bar{z}+y\right)}{\tau m r},
$$

then we have

$$
\bar{u}_{i}^{\varepsilon}(0)=2 \frac{u_{i}^{\varepsilon}(y)}{\tau m r}=\frac{2 \sup _{\partial \Omega} f_{i}}{\tau r},
$$

and

$$
0 \leq \Delta \bar{u}_{i}^{\varepsilon}(\bar{z}) \leq \frac{\tau m r}{2} \bar{u}_{i}^{\varepsilon}(\bar{z}) \sum_{j \neq i} \frac{1}{\varepsilon^{2}} H\left(u_{j}^{\varepsilon}\right)\left(\frac{\tau m r}{2} \bar{z}+y\right) \quad \text { for } \bar{z} \in \mathcal{B}_{1}(0)
$$

where

$$
\frac{\tau m r}{2} \bar{u}_{i}^{\varepsilon}(\bar{z}) \sum_{j \neq i} \frac{1}{\varepsilon^{2}} H\left(u_{j}^{\varepsilon}\right)\left(\frac{\tau m r}{2} \bar{z}+y\right) \leq \frac{C e^{-c \varepsilon^{-\frac{1}{2}}}}{\varepsilon^{2}} .
$$

Then, by the Harnack inequality (see e.g. Theorem 4.3 in [3]), we get

$$
\sup _{\mathcal{B}_{\frac{1}{2}}(0)} \bar{u}_{i}^{\varepsilon} \leq C_{n}\left(\inf _{\mathcal{B}_{\frac{1}{2}}(0)} \bar{u}_{i}^{\varepsilon}+\frac{C e^{-c \varepsilon^{-\frac{1}{2}}}}{\varepsilon^{2}}\right) \leq C_{n}\left(\frac{2 \sup _{\partial \Omega} f_{i}}{\tau r}+\frac{C e^{-c \varepsilon^{-\frac{1}{2}}}}{\varepsilon^{2}}\right) \leq \frac{C}{r} .
$$

Lipschitz estimates then imply that $\left|\nabla \bar{u}_{i}^{\varepsilon}\right| \leq C / r$ in $\mathcal{B}_{\frac{1}{2}}(0)$ and 5.7 follows.

Further, 5.9 implies (5.8).

The next lemma says that in a $\rho$-strip of size 1 of support of the $f_{j}$ 's, the function $u_{i}^{\varepsilon}, i \neq j$, decays to 0 exponentially.

Lemma 5.5. Assume 2.8). Let $\left(u_{1}^{\varepsilon}, \ldots, u_{K}^{\varepsilon}\right)$ be a viscosity solution of the problem (2.4). For $j=1, \ldots, K, \sigma>0$, let $\bar{\Gamma}_{j}^{\sigma}:=\left\{f_{j} \geq \sigma\right\} \subset \Omega^{c}$. Then on the sets

$$
\left\{x \in \Omega: d_{\rho}\left(x, \bar{\Gamma}_{j}^{\sigma}\right) \leq 1-r\right\}, \quad 0<r<1
$$


we have

$$
u_{i}^{\varepsilon} \leq C e^{-\frac{c \sigma^{\alpha} r^{\beta}}{\varepsilon}}, \text { for } i \neq j
$$

for some positive $\alpha$ and $\beta$ depending on the structure of $H$ ( $p$ and $q)$ and the modulus of continuity of $f_{j}$.

Proof. Let $\bar{x} \in \Omega$ and $y \in \bar{\Gamma}_{j}^{\sigma}$ be such that $d_{\rho}(\bar{x}, y) \leq 1-r$. We want to estimate $H\left(u_{j}^{\varepsilon}\right)(x)$, for any $x \in \mathcal{B}_{\frac{r}{2}}(\bar{x})$. Let $x \in \mathcal{B}_{\frac{r}{2}}(\bar{x})$, then

$$
d_{\rho}(x, y) \leq 1-\frac{r}{2}
$$

Let us first consider the case $H$ defined as in (2.6). We have

$$
H\left(u_{j}^{\varepsilon}\right)(x)=\sup _{\mathcal{B}_{1}(x)} u_{j}^{\varepsilon} \geq f_{j}(y) \geq \sigma .
$$

Next, let us turn to the case $H$ defined as in (2.5). Let $r_{0}:=\min \left\{\sigma^{\gamma}, r / 4\right\}$, for some $\gamma$ depending on the modulus of continuity of $f_{j}$, then $f_{j} \geq \sigma / 2$ in the set $\mathcal{B}_{r_{0}}(y) \cap \operatorname{supp} f_{j}$. Moreover, remark that from (5.10) and $r_{0} \leq r / 4$, we have

$$
\mathcal{B}_{r_{0}}(y) \cap \operatorname{supp} f_{j} \subset \mathcal{B}_{\frac{r}{4}}(y) \subset \mathcal{B}_{\frac{r}{2}}(y) \subset \mathcal{B}_{1}(x),
$$

and for any $z \in \mathcal{B}_{r_{0}}(y) \cap \operatorname{supp} f_{j}$

$$
\rho(x-z) \leq \rho(x-y)+\rho(y-z) \leq 1-\frac{r}{2}+r_{0} \leq 1-\frac{r}{4}
$$

Therefore, using in addition (2.7), and that, by (2.8), $\left|\mathcal{B}_{r_{0}}(y) \cap \operatorname{supp} f_{j}\right| \geq c\left|\mathcal{B}_{r_{0}}(y)\right|$, we get

$$
\begin{aligned}
H\left(u_{j}^{\varepsilon}\right)(x) & =\int_{\mathcal{B}_{1}(x)}\left(u_{j}^{\varepsilon}\right)^{p}(z) \varphi(\rho(x-z)) d z \\
& \geq \int_{\mathcal{B}_{r_{0}}(y) \cap \operatorname{supp} f_{j}}\left(u_{j}^{\varepsilon}\right)^{p}(z)(1-\rho(x-z))^{q} d z \\
& \geq \int_{\mathcal{B}_{r_{0}}(y) \cap \operatorname{supp} f_{j}}\left(f_{j}\right)^{p}(z) C\left(\frac{r}{4}\right)^{q} d z \\
& \geq C \sigma^{p} r_{0}^{\bar{\beta}},
\end{aligned}
$$

where $\bar{\beta}$ depends on $q$ and on the dimension $n$. 
Then, for $H$ defined as in (2.5) or (2.6), the function $u_{i}^{\varepsilon}, i \neq j$ (which is eventually zero in $\left.B_{\frac{r}{2}}(\bar{x}) \cap \Omega^{c}\right)$ is subsolution of

$$
\Delta u_{i}^{\varepsilon} \geq u_{i}^{\varepsilon} \frac{C \sigma^{p} r_{0}^{\bar{\beta}}}{\varepsilon^{2}}
$$

in $B_{\frac{r}{2}}(\bar{x})$, where $p=1$ and $\bar{\beta}=0$ in the case 2.6). The conclusion follows as in Lemma 5.3 .

The following corollary is a consequence of Lemma 5.3. Corollary 5.4 and Lemma 5.5 .

Corollary 5.6. Assume 2.8. Let $\left(u_{1}^{\varepsilon}, \ldots, u_{K}^{\varepsilon}\right)$ be a viscosity solution of the problem (2.4).

Then, there exists a subsequence $\left(u_{1}^{\varepsilon_{1}}, \ldots, u_{K}^{\varepsilon_{l}}\right)$ and continuous functions $\left(u_{1}, \ldots, u_{K}\right)$ such that,

$$
\left(u_{1}^{\varepsilon_{l}}, \ldots, u_{K}^{\varepsilon_{l}}\right) \rightarrow\left(u_{1}, \ldots, u_{K}\right) \quad \text { as } l \rightarrow+\infty, \quad \text { a.e. in } \Omega
$$

and the convergence of $u_{i}^{\varepsilon_{l}}$ to $u_{i}$ is locally uniform in the set $\left\{x \in \Omega: d_{\rho}\left(x, \operatorname{supp} f_{j}\right)>1, j \neq i\right\}$. Moreover, we have:

i) the $u_{i}$ 's are locally Lipschitz continuous in $\Omega$ and have disjoint supports, in particular

$$
u_{i} \equiv 0 \quad \text { in the set } \quad\left\{x \in \Omega \mid d_{\rho}\left(x, \operatorname{supp} u_{j}\right) \leq 1\right\} \quad \text { for any } j \neq i \text {. }
$$

ii) $\Delta u_{i}=0$ when $u_{i}>0$.

Proof. Fix an index $i=1, \ldots, K$. Let us denote

$$
\Omega_{i}:=\left\{x \in \Omega \mid d_{\rho}\left(x, \operatorname{supp} f_{j}\right)>1 \text { for any } j \neq i\right\}
$$

and

$$
B_{i}:=\Omega \backslash \bar{\Omega}_{i}
$$

Claim 1: $u_{i}^{\varepsilon}(x) \rightarrow 0$ as $\varepsilon \rightarrow 0$ for any $x \in B_{i}$.

Indeed, let $x_{0}$ belong to $B_{i}$, then there exists $j \neq i$ such that $d_{\rho}\left(x_{0}, \operatorname{supp} f_{j}\right)<1$. Remark that

$$
\left\{x \in \Omega \mid d_{\rho}\left(x, \operatorname{supp} f_{j}\right)<1\right\} \subset \cup_{r, \sigma>0}\left\{x \in \Omega \mid d_{\rho}\left(x, \bar{\Gamma}_{j}^{\sigma}\right) \leq 1-r\right\},
$$

where $\bar{\Gamma}_{j}^{\sigma}=\left\{f_{j} \geq \sigma\right\}$. Therefore, there exist $r, \sigma>0$ such that $x_{0} \in\left\{x \in \Omega \mid d_{\rho}\left(x, \bar{\Gamma}_{j}^{\sigma}\right) \leq 1-r\right\}$, and by Lemma 5.5 we have that $u_{i}^{\varepsilon}\left(x_{0}\right) \leq C e^{-\frac{c \sigma^{\alpha} r^{\beta}}{\varepsilon}}$, for some $\alpha, \beta>0$. Claim 1 follows. 
Claim 2: there exists a subsequence $\left(u_{i}^{\varepsilon_{l}}\right)_{l}$ locally uniformly convergent in $\Omega_{i}$ as $l \rightarrow+\infty$ to a locally Lipschitz continuous function $u_{i}$.

Fix, $0<r<1$, and define

$$
\Omega_{i}^{r}:=\left\{x \in \Omega_{i} \mid d_{\rho}(x, \partial \Omega)>2 r, d_{\rho}\left(x, \operatorname{supp} f_{j}\right) \geq 1+\tau r \text { for any } j \neq i\right\}
$$

Fix $\theta<\frac{1}{2 \alpha}$ and set $\sigma_{\varepsilon}=\varepsilon^{\theta}>0$ and consider $\tau, \alpha$ and $\beta$ as given by Lemma 5.3. Since $\varepsilon=\sigma_{\varepsilon}^{2 \alpha} \sigma_{\varepsilon}^{\frac{1}{\theta}-2 \alpha}=\sigma_{\varepsilon}^{2 \alpha} \varepsilon^{\theta\left(\frac{1}{\theta}-2 \alpha\right)}$ and $\frac{1}{\theta}-2 \alpha>0$, we can fix $\varepsilon_{0}=\varepsilon_{0}(r)$ so small that for any $\varepsilon<\varepsilon_{0}$ we have that $\varepsilon \leq \sigma_{\varepsilon}^{2 \alpha} r^{2 \beta}$. Then, by Corollary 5.4 , the functions

$$
v_{i}^{\varepsilon}:=\left(u_{i}^{\varepsilon}-\sigma_{\varepsilon}\right)_{+}=\left(u_{i}^{\varepsilon}-\varepsilon^{\theta}\right)_{+}
$$

are Lipschitz continuous in $\Omega_{i}^{r}$. Indeed, when $u_{i}^{\varepsilon}(x)<\varepsilon^{\theta}$, then $v_{i}^{\varepsilon}(x)=0$. Next, let $x$ such that $u_{i}^{\varepsilon}(x)>\varepsilon^{\theta}$, then $\nabla v_{i}^{\varepsilon}(x)=\nabla u_{i}^{\varepsilon}(x)$. Set $\sigma=u_{i}^{\varepsilon}(x)$, then at those points $x$, we have that $d_{\rho}\left(x, \operatorname{supp} f_{j}\right) \geq 1+\tau r \geq 1+m \tau r$, where $m=\sigma / \sup _{\partial \Omega} f_{i} \leq 1$. Moreover, $d_{\rho}(x, \partial \Omega)>2 r$ and $\varepsilon \leq \sigma_{\varepsilon}^{2 \alpha} r^{2 \beta} \leq \sigma^{2 \alpha} r^{2 \beta}$. We can therefore apply Corollary 5.4 and we get that

$$
\left|\nabla u_{i}^{\varepsilon}(x)\right| \leq \frac{C_{0}}{r}
$$

This concludes the proof that the functions $v_{i}^{\varepsilon}$ are Lipschitz continuous in $\Omega_{i}^{r}$. Therefore, we can extract a subsequence $\left(v_{i}^{\varepsilon_{l}}\right)_{l}$ uniformly convergent to a Lipschitz continuous function $u_{i}$ in $\Omega_{i}^{r}$ as $l \rightarrow+\infty$. By the definition of the $v_{i}$ 's, this implies that there exists a subsequence $\left(u_{i}^{\varepsilon_{l}}\right)_{l}$ uniformly convergent to the same function $u_{i}$ in $\Omega_{i}^{r}$ as $l \rightarrow+\infty$. Taking $r \rightarrow 0$ and using a diagonalization argument, we can find a subsequence of $\left(u_{i}^{\varepsilon}\right)_{\varepsilon}$ converging locally uniformly to a Lipschitz function $u_{i}$ in $\Omega_{i}$. This ends the proof of Claim 2 .

Claims 1 and 2 yield the convergence, up to a subsequence, of $u_{i}^{\varepsilon}$ to a continuous function $u_{i}$ which is locally Lipchitz in both $\Omega_{i}$ and $B_{i}$. The fact that the supports of the limit functions are at distance greater or equal than 1, is a consequence of Claims 1 and 2 and Lemma 5.3 . Moreover, from the proof of Claim 2 and Corollary 5.4, we infer that the limit function $u_{i}$ is harmonic inside its support, i.e. (ii). To conlude the proof of (i), we just need to prove that $u_{i}$ is Lipschitz in a neighborhood of points belonging to $\partial B_{i}=\partial \Omega_{i} \cap \Omega$. Let $x_{0} \in \partial \Omega_{i} \cap \Omega$, then 
from Claim $1, u_{i}\left(x_{0}\right)=0$. If $x_{0} \notin \partial\left\{u_{i}>0\right\}$, then in a neighborhood of $x_{0}, u_{i} \equiv 0$ and of course it is Lipschitz there. On the other hand, if $x_{0} \in \partial\left\{u_{i}>0\right\}$, then, since there exists an exterior $\rho$-tangent ball of radius 1 at any point of $\partial \Omega_{i} \cap \Omega$ and $u_{i}$ is harmonic inside its support, a barrier argument implies that there exist $r_{0}, C>0$ such that $0 \leq u_{i}(x)=u_{i}(x)-u_{i}\left(x_{0}\right) \leq C\left|x-x_{0}\right|$ for any $x \in B_{r_{0}}\left(x_{0}\right)$. This concludes the proof of (i).

This concludes the proof of the corollary.

\section{A SEMICONVEXIty PROPERTy OF THE FREE BOUNDARIES}

Let $\left(u_{1}, \ldots, u_{K}\right)$ be the limit of a convergent subsequence of $\left(u_{1}^{\varepsilon}, \ldots, u_{K}^{\varepsilon}\right)$, whose existence is guaranteed by Corollary 5.6. For $i=1, \ldots, K$, let us denote

$$
S\left(u_{i}\right):=\left\{x \in \Omega: u_{i}>0\right\}
$$

(In the next sections, for simplicity this set will be represented by $S_{i}$.) Then the sets $S\left(u_{i}\right)$ have the following semiconvexity property:

Lemma 6.1. Given $S\left(u_{i}\right)$ consider

$$
T\left(u_{i}\right)=\left\{x \in \Omega: d_{\rho}\left(x, S\left(u_{i}\right)\right) \geq 1\right\}
$$

and

$$
S^{*}\left(u_{i}\right)=\left\{x \in \Omega: d_{\rho}\left(x, T\left(u_{i}\right)\right)>1\right\}
$$

Then $\partial S\left(u_{i}\right) \subset \partial S^{*}\left(u_{i}\right)$.

Proof. We have that $S^{*}\left(u_{i}\right) \supset S\left(u_{i}\right)$. To prove the desired inclusion, for $\sigma>0$ consider the sets

$$
\begin{gathered}
S_{\sigma}\left(u_{i}\right):=\left\{x \in \Omega: u_{i}>\sigma\right\} \\
T_{\sigma}\left(u_{i}\right):=\left\{x \in \Omega: d_{\rho}\left(x, S_{\sigma}\left(u_{i}\right)\right) \geq 1\right\}
\end{gathered}
$$

and

$$
S_{\sigma}^{*}\left(u_{i}\right):=\left\{x \in \Omega: d_{\rho}\left(x, T_{\sigma}\left(u_{i}\right)\right)>1\right\} .
$$


Notice that, the union of $\rho$-balls centered at points in $S_{\sigma}\left(u_{i}\right)$ coincides with the union of $\rho$-balls centered at points in $S_{\sigma}^{*}\left(u_{i}\right)$, i.e.

a) $\left(T_{\sigma}\left(u_{i}\right)\right)^{c}=\cup \mathcal{B}_{1}(x)$ for $x \in S_{\sigma}\left(u_{i}\right)$ and

b) $\left(T_{\sigma}\left(u_{i}\right)\right)^{c}=\cup \mathcal{B}_{1}(x)$ for $x \in S_{\sigma}^{*}\left(u_{i}\right)$.

If $x \in S_{\sigma}\left(u_{i}\right)$, from (i) of Corollary 5.6 we have that $d_{\rho}\left(x, \operatorname{supp} f_{j}\right)>1$ for $j \neq i$, and the locally uniform convergence of $u_{i}^{\varepsilon}$ to $u_{i}$ and Lemma 5.3 imply that, up to subsequences, $u_{j}^{\varepsilon} \leq C e^{-\frac{c \sigma^{\alpha} r^{\beta}}{\varepsilon}}$ in $\mathcal{B}_{1}(x)$, where $2 r=\min \left\{d_{\rho}\left(x, \operatorname{supp} f_{i}\right), C\left(d_{\rho}\left(x, \operatorname{supp} f_{j}\right)-1\right)\right\}$. Now, the set where $u_{j}^{\varepsilon}$ decays is the same if we had considered $x \in S_{\sigma}^{*}\left(u_{i}\right)$, since from (a) and (b) we have

$$
\cup_{x \in S_{\sigma}\left(u_{i}\right)} \mathcal{B}_{1}(x)=\cup_{x \in S_{\sigma}^{*}\left(u_{i}\right)} \mathcal{B}_{1}(x) .
$$

Therefore $\frac{H\left(u_{j}^{\varepsilon}\right)}{\varepsilon^{2}}$ goes to zero as $\varepsilon$ goes to zero in $S_{\sigma}^{*}\left(u_{i}\right)$. It follows that $\Delta u_{i} \equiv 0$ in $S_{\sigma}^{*}\left(u_{i}\right)$, if $S_{\sigma}^{*}\left(u_{i}\right)$ is not empty. Now, if $A$ is a connected component of $S_{\sigma}\left(u_{i}\right)$, then there exists a connected component $A^{*}$ of $S_{\sigma}^{*}\left(u_{i}\right)$ such that $A \subset A^{*}$. Since $u_{i}$ is harmonic and non-negative in $A^{*}$, the strong maximum principle implies that $u_{i}>0$ in all $A^{*}$, that is $A^{*} \subset A$. We conclude that $A=A^{*}$. Therefore, any connected component of $S_{\sigma}\left(u_{i}\right)$ is equal to a connected component of $S_{\sigma}^{*}\left(u_{i}\right)$. Passing to the limit as $\sigma \rightarrow 0$, we obtain that any connected component of $S\left(u_{i}\right)$ is equal to a connected component of $S^{*}\left(u_{i}\right)$. In particular, $\partial S\left(u_{i}\right) \subset \partial S^{*}\left(u_{i}\right)$.

From Lemma 6.1 we can conclude that the sets $S\left(u_{i}\right)$ have a tangent $\rho$-ball of radius 1 from outside at any point of the boundary, as stated in the following corollary.

Corollary 6.2. If $x_{0} \in \partial S\left(u_{i}\right) \cap \Omega$ there is an exterior tangent ball, $\mathcal{B}_{1}(y)$ at $x_{0}$, in the sense that for $x \in \mathcal{B}_{1}(y) \cap \mathcal{B}_{1}\left(x_{0}\right)$, all $u_{j}(x) \equiv 0$ (including $u_{i}$ ).

The following two lemmas about the distance function may be known in the literature and we provide the proof here for the reader's convenience. 
Lemma 6.3. Let $S$ be a closed set. Let $d_{\rho}(\cdot, S)$ denote the $\rho$-distance function from $S$. Then, in the set $\left\{x: d_{\rho}(x, S)>0\right\}, d_{\rho}(\cdot, S)$ satisfies in the viscosity sense

$$
\Delta d_{\rho}(\cdot, S) \leq \frac{C}{d_{\rho}(\cdot, S)}
$$

where $C$ is a constant depending on $n,\left\|D d_{\rho}(\cdot, S)\right\|_{L^{\infty}}$ and the constant $A$ given in (2.1).

Proof. We first prove that there exists a smooth tangent function from above at any point of the graph of $d_{\rho}(\cdot, S)$ in the set $\left\{d_{\rho}(\cdot, S)>0\right\}$. For simplicity we will write $d_{S}(\cdot)$ instead of $d_{\rho}(\cdot, S)$. Let $y_{0}$ be a point in the complementary of $S$. Let $x \in \partial S$ be a point where $y_{0}$ realizes the distance from $S$. Assume, without loss of generality, that $x=0$. Then $d_{\rho}\left(y_{0}, 0\right)=\rho\left(y_{0}\right)=d_{S}\left(y_{0}\right)$. In particular, the ball $\mathcal{B}_{\rho\left(y_{0}\right)}\left(y_{0}\right)$ is contained in $S^{c}$ and tangent to $S$ at 0 . For any $y \in \mathcal{B}_{\rho\left(y_{0}\right)}\left(y_{0}\right)$, we have that $d_{S}(y) \leq d_{\rho}(y, 0)=\rho(y)$, therefore the cone, graph of the function $y \rightarrow \rho(y)$, is tangent from above to the graph of $d_{S}(\cdot)$ at $\left(y_{0}, d_{S}\left(y_{0}\right)\right)$.

Next, let $\psi$ be a test function touching from below $d_{S}(\cdot)$ at $y_{0}$, then $\psi$ touches from below the function $\rho(y)$ at $y_{0}$. In particular, $\Delta \psi\left(y_{0}\right) \leq \Delta \rho\left(y_{0}\right)$. Let us compute $\Delta \rho$. Using (2.1), we get

$$
D^{2}(\rho)=\frac{1}{\rho} D^{2}\left(\frac{1}{2} \rho^{2}\right)-\frac{D \rho \otimes D \rho}{\rho} \leq \frac{1}{\rho}\left(A I_{n}-D \rho \otimes D \rho\right)
$$

which gives

$$
\Delta \rho \leq \frac{C}{\rho}
$$

In particular,

$$
\Delta \psi\left(y_{0}\right) \leq \frac{C}{\rho\left(y_{0}\right)}=\frac{C}{d_{S}\left(y_{0}\right)}
$$

This concludes the proof.

Lemma 6.4. Let $S$ be a closed and bounded set. Let us denote by $d_{\rho}(\cdot, S)$ the $\rho$-distance function from $S$ and by $(S)_{1}$ the set at $\rho$-distance 1 from $S$. Then $(S)_{1}$ has finite perimeter.

Proof. For simplicity we will write $d_{S}(\cdot)$ instead of $d_{\rho}(\cdot, S)$, as in the previous lemma and first we present an heuristic proof integrating $\Delta d_{S}^{2}$ over the set $\left\{0<d_{S}<1\right\}$. Since $\left|D d_{S}\right|$ is bounded, 
from Lemma 6.3 , we see that

$$
\Delta d_{S}^{2}=2\left|D d_{S}\right|^{2}+2 d_{S} \Delta d_{S} \leq C
$$

Therefore, integrating $\Delta d_{S}^{2}$, we get

$$
\begin{aligned}
C & \geq \int_{\left\{0<d_{S}<1\right\}} \Delta d_{S}^{2} d x=\int_{\left\{d_{S}=0\right\}} 2 d_{S} D d_{S} \cdot n d \mathcal{H}^{n-1}+\int_{\left\{d_{S}=1\right\}} 2 d_{S} D d_{S} \cdot n d \mathcal{H}^{n-1} \\
& =\int_{\left\{d_{S}=1\right\}} 2 D d_{S} \cdot n d \mathcal{H}^{n-1} \geq c \int_{\left\{d_{S}=1\right\}} d \mathcal{H}^{n-1}=c \mathcal{H}^{n-1}\left(\left\{d_{S}=1\right\}\right),
\end{aligned}
$$

where $n=D d_{S} /\left|D d_{S}\right|$ is the unit exterior normal. This provides un upper bound for $\mathcal{H}^{n-1}\left(\left\{d_{S}=\right.\right.$ 1\}) and concludes the heuristic proof.

To make the argument precise, we need to correct the regularity problem over the boundary. For that, consider a smooth function $\eta$ with compact support in $(0,1)$ such that $0 \leq \eta(\xi) \leq \xi$ for any $\xi \in[0,1], \eta(\xi)=\xi$ for $\xi \in[\delta, 1-\delta],\left|\eta^{\prime}\right| \leq c$ on $(0,1-\delta)$ and $\eta^{\prime}(\xi) \leq-c / \delta$ for $\xi \in(1-\delta, 1)$, where $\delta>0$ is a small parameter. Then, in a weak sense we have

$$
\operatorname{div}\left(\eta\left(d_{S}\right) D d_{S}\right)=\eta^{\prime}\left(d_{S}\right)\left|D d_{S}\right|^{2}+\eta\left(d_{S}\right) \Delta d_{S}
$$

Moreover, from Lemma 6.3, in the set $\left\{0<d_{S}<1\right\}$ we have

$$
\eta\left(d_{S}\right) \Delta d_{S} \leq \eta\left(d_{S}\right) \frac{C}{d_{S}} \leq C
$$

in the viscosity sense and therefore in the distributional sense (see, e.g., 24 for the equivalence between viscosity solutions and weak solutions). Therefore, since $\eta\left(d_{S}\right)$ is a function with compact support in $\left\{0<d_{S}<1\right\}$, we get

$$
\begin{aligned}
0 & =\int_{\left\{0<d_{S}<1\right\}} \operatorname{div}\left(\eta\left(d_{S}\right) D d_{S}\right) \leq \int_{\left\{0<d_{S}<1\right\}} \eta^{\prime}\left(d_{S}\right)\left|D d_{S}\right|^{2} d x+C \\
& =\int_{\left\{0<d_{S}<1-\delta\right\}} \eta^{\prime}\left(d_{S}\right)\left|D d_{S}\right|^{2} d x+\int_{\left\{1-\delta<d_{S}<1\right\}} \eta^{\prime}\left(d_{S}\right)\left|D d_{S}\right|^{2} d x+C \\
& \leq \int_{\left\{1-\delta<d_{S}<1\right\}} \eta^{\prime}\left(d_{S}\right)\left|D d_{S}\right|^{2} d x+C \\
& \leq-\frac{c}{\delta} \int_{\left\{1-\delta<d_{S}<1\right\}}\left|D d_{S}\right|^{2} d x+C .
\end{aligned}
$$

Now, using the coarea formula and the inequalities above, we get

$$
\frac{1}{\delta} \int_{1-\delta}^{1} \mathcal{H}^{n-1}\left(\left\{d_{S}=t\right\}\right) d t=\frac{1}{\delta} \int_{\left\{1-\delta<d_{S}<1\right\}}\left|D d_{S}\right|^{2} d x \leq C .
$$


Finally, taking the limit as $\delta \rightarrow 0^{+}$and using the lower semicontinuity of the perimeter with respect to the convergence in measure, we infer that

$$
\operatorname{Per}\left(\left\{d_{S}=1\right\}\right) \leq \liminf _{\delta \rightarrow 0^{+}} \frac{1}{\delta} \int_{1-\delta}^{1} \mathcal{H}^{n-1}\left(\left\{d_{S}=t\right\}\right) d t \leq C .
$$

This concludes the proof of the lemma.

Corollary 6.5. The sets $S\left(u_{i}\right), i=1, \ldots, K$ have finite perimeter.

Proof. The corollary is an immediate consequence of Lemma 6.1 and Lemma 6.4.

\section{A sharp Characterization of the interfaces}

In Section 5 we proved that the supports of the limit functions $u_{i}$ 's are at distance at least 1, one from each other (see Corollary 5.6). In this section we will prove that they are exactly at distance 1, as stated in the following theorem.

Theorem 7.1. Assume (2.8) with $p=1$ in 2.5. Let $\left(u_{1}^{\varepsilon}, \ldots, u_{K}^{\varepsilon}\right)$ be a viscosity solution of the problem (2.4) and $\left(u_{1}, \ldots, u_{K}\right)$ the limit as $\varepsilon \rightarrow 0$ of a convergent subsequence. Let $x_{0} \in \partial\left\{u_{i}>0\right\} \cap \Omega$, then there exists $j \neq i$ such that

$$
\overline{\mathcal{B}_{1}\left(x_{0}\right)} \cap \partial\left\{u_{j}>0\right\} \neq \emptyset
$$

Proof. It is enough to prove the theorem for a point $x_{0}$ for which $\partial S\left(u_{i}\right)$ has a tangent $\rho$-ball from inside, since such points are dense on $\partial S\left(u_{i}\right)$. Indeed, let $x$ be any point of $\partial S\left(u_{i}\right)$. Let us consider a sequence of points $\left(x_{k}\right)$ contained in $S\left(u_{i}\right)$ and converging to $x$ as $k \rightarrow \infty$. Let $d_{k}$ be the $\rho$-distance of $x_{k}$ from $\partial S\left(u_{i}\right)$. Then the $\rho$-balls $\mathcal{B}_{d_{k}}\left(x_{k}\right)$ are contained in $S\left(u_{i}\right)$ and there exist points $y_{k} \in \partial S\left(u_{i}\right) \cap \mathcal{B}_{d_{k}}\left(x_{k}\right)$ where the $x_{k}$ 's realize the distance from $\partial S\left(u_{i}\right)$. The sequence $\left(y_{k}\right)$ is a sequence of points of $\partial S\left(u_{i}\right)$ that have a tangent $\rho$-ball from the inside and converges to $x$.

Next, remark that from (b) in Corollary 5.6, we have that $d_{\rho}\left(x_{0}, \operatorname{supp} f_{j}\right) \geq 1$ for any $j \neq i$. If there is a $j$ such that $d_{\rho}\left(x_{0}, \operatorname{supp} f_{j}\right)=1$, then 7.1 is obviously true. Therefore, we can assume 
that $d_{\rho}\left(x_{0}, \operatorname{supp} f_{j}\right)>1$ for any $j \neq i$. Then, for small $S>0$ we have that $\mathcal{B}_{1+S}\left(x_{0}\right) \cap \operatorname{supp} f_{j}=$ $\emptyset$ and from (2.4), we know that

$$
\Delta u_{j}^{\varepsilon} \geq \frac{1}{\varepsilon^{2}} u_{j}^{\varepsilon} \sum_{k \neq j} H\left(u_{k}^{\varepsilon}\right) \quad \text { in } \mathcal{B}_{1+S}\left(x_{0}\right) .
$$

We divide the proof in two cases.

a) $H(u)(x)=\int_{\mathcal{B}_{1}(x)} u(y) \varphi(\rho(x-y)) \mathrm{d} y$

and

b) $H(u)(x)=\sup _{y \in \mathcal{B}_{1}(x)} u(y)$.

Proof of case a): Let $S\left(u_{i}\right)=\left\{x \in \Omega: u_{i}>0\right\}$ as in 6.1. Let $\mathcal{B}_{S}$ be a small $\rho$-ball centered at $x_{0} \in \partial S\left(u_{i}\right)$. Then, as a measure, as $\varepsilon \rightarrow 0$, up to subsequence

$$
\left.\left.\Delta u_{i}^{\varepsilon}\right|_{\mathcal{B}_{S}\left(x_{0}\right)} \longrightarrow \Delta u_{i}\right|_{\mathcal{B}_{S}\left(x_{0}\right)}
$$

(that has strictly positive mass, since $u_{i}$ is not harmonic in $\mathcal{B}_{S}\left(x_{0}\right)$ ).

We bound by below

$$
\int_{\mathcal{B}_{1+S}\left(x_{0}\right)} \sum_{j \neq i} \Delta u_{j}^{\varepsilon} d x \quad \text { by } \quad \int_{\mathcal{B}_{S}\left(x_{0}\right)} \Delta u_{i}^{\varepsilon} d x \text {. }
$$

Indeed

$$
\begin{aligned}
\varepsilon^{2} \int_{\mathcal{B}_{S}\left(x_{0}\right)} \Delta u_{i}^{\varepsilon}(x) d x & =\sum_{j \neq i} \int_{\mathcal{B}_{S}\left(x_{0}\right)} \int_{\mathcal{B}_{1}(x)} u_{i}^{\varepsilon}(x) \varphi(\rho(x-y)) u_{j}^{\varepsilon}(y) d y d x \\
& =\sum_{j \neq i} \iint_{\mathcal{B}_{S}\left(x_{0}\right) \times \mathcal{B}_{1+S}\left(x_{0}\right)} u_{i}^{\varepsilon}(x) \chi_{[0,1]}(\rho(x-y)) \varphi(\rho(x-y)) u_{j}^{\varepsilon}(y) d x d y \\
& \leq \sum_{j \neq i} \iint_{\mathcal{B}_{2+S}\left(x_{0}\right) \times \mathcal{B}_{1+S}\left(x_{0}\right)} u_{i}^{\varepsilon}(x) \chi_{[0,1]}(\rho(x-y)) \varphi(\rho(x-y)) u_{j}^{\varepsilon}(y) d x d y \\
& =\sum_{j \neq i} \int_{\mathcal{B}_{1+S}\left(x_{0}\right)} \int_{\mathcal{B}_{1}(y)} u_{i}^{\varepsilon}(x) \varphi(\rho(x-y)) u_{j}^{\varepsilon}(y) d x d y \\
& \leq \varepsilon^{2} \sum_{j \neq i} \int_{\mathcal{B}_{1+S}\left(x_{0}\right)} \Delta u_{j}^{\varepsilon}(y) d y
\end{aligned}
$$

where $\chi_{[0,1]}$ is the indicator function of the set $[0,1]$. 
Therefore, for any small positive $S$, taking the limit in $\varepsilon$ we get

$$
\int_{\mathcal{B}_{1+S}\left(x_{0}\right)} \sum_{j \neq i} \Delta u_{j} \geq \int_{\mathcal{B}_{S}\left(x_{0}\right)} \Delta u_{i}>0
$$

which implies that there exists $j \neq i$ such that $u_{j}$ cannot be identical equal to zero in $\mathcal{B}_{1+S}\left(x_{0}\right)$. Since $S$ small is arbitrary, the result follows.

The case b) is more involved. We may assume $x_{0}=0$. Let $y_{0}$ be such that $\mathcal{B}_{\mu}\left(y_{0}\right) \subset$ $S\left(u_{i}\right)$ and $0 \in \partial \mathcal{B}_{\mu}\left(y_{0}\right)$. By Corollary 6.2 we know that there exists a $\rho$-ball $\mathcal{B}_{1}\left(y_{1}\right)$ such that $\mathcal{B}_{1}\left(y_{1}\right) \cap S\left(u_{i}\right)=\emptyset$ and $0 \in \partial \mathcal{B}_{1}\left(y_{1}\right)$.

Let us first prove two claims.

Claim 1: There exists $\mu^{\prime}<\mu$ and $C_{1}>0$ such that in the annulus $\left\{\mu^{\prime}<\rho\left(x-y_{0}\right)<\mu\right\}$ we have

$$
u_{i}(x) \geq C_{1} d_{\rho}\left(x, \partial \mathcal{B}_{\mu}\left(y_{0}\right)\right)
$$

Since any $\rho$-ball $\mathcal{B}$ satisfies the uniform interior ball condition, for any point $\bar{x} \in \partial \mathcal{B}_{\mu}\left(y_{0}\right)$ there exists an Euclidian ball $B_{R_{0}}\left(z_{0}\right)$ of radius $R_{0}$ independent of $\bar{x}$ contained in $\mathcal{B}_{\mu}\left(y_{0}\right)$ and tangent to $\partial \mathcal{B}_{\mu}\left(y_{0}\right)$ at $\bar{x}$. Let $m>0$ be the infimum of $u_{i}$ on the set $\left\{x \in \mathcal{B}_{\mu}\left(y_{0}\right) \mid d\left(x, \partial \mathcal{B}_{\mu}\left(y_{0}\right)\right) \geq R_{0} / 2\right\}$, where $d$ is the Euclidian distance function, and let $\phi$ be the solution of

$$
\left\{\begin{array}{lll}
\Delta \phi=0 & \text { in } & \left\{\frac{R_{0}}{2}<\left|x-z_{0}\right|<R_{0}\right\} \\
\phi=0 & \text { on } & \partial B_{R_{0}}\left(z_{0}\right) \\
\phi=m & \text { on } & \partial B_{\frac{R_{0}}{2}}\left(z_{0}\right)
\end{array}\right.
$$

i.e., for $n \geq 3$,

$$
\phi(x)=C(n) m\left(\frac{R_{0}^{n-2}}{\left|x-z_{0}\right|^{n-2}}-1\right) .
$$

Since $u_{i}$ is harmonic in $\mathcal{B}_{\mu}\left(y_{0}\right)$ and $u_{i} \geq \phi$ on $\partial B_{R_{0}}\left(z_{0}\right) \cup \partial B_{\frac{R_{0}}{2}}\left(z_{0}\right)$, by comparison principle $u_{i} \geq \phi$ in $\left\{\frac{R_{0}}{2}<\left|x-z_{0}\right|<R_{0}\right\}$. In particular, for any $x \in\left\{\frac{R_{0}}{2}<\left|x-z_{0}\right|<R_{0}\right\}$ and belonging to the segment between $z_{0}$ and $\bar{x}$, using that $\phi$ is convex in the radial direction,

$$
\left.\frac{\partial \phi}{\partial \nu_{i}}\right|_{\partial B_{R_{0}}\left(z_{0}\right)}=\frac{C(n)(n-2) m}{R_{0}}
$$


where $\nu_{i}$ is the interior normal at $\partial B_{R_{0}}\left(z_{0}\right)$, and (2.2), we get

$$
u_{i}(x) \geq \frac{C(n)(n-2) m}{R_{0}} d\left(x, \partial B_{R_{0}}\left(z_{0}\right)\right)=C\left(n, R_{0}\right) m d\left(x, \partial \mathcal{B}_{\mu}\left(y_{0}\right)\right) \geq C_{1} d_{\rho}\left(x, \partial \mathcal{B}_{\mu}\left(y_{0}\right)\right) .
$$

Therefore, letting $\bar{x}$ vary in $\partial \mathcal{B}_{\mu}\left(y_{0}\right)$ we get

$$
u_{i}(x) \geq C_{1} d_{\rho}\left(x, \partial \mathcal{B}_{\mu}\left(y_{0}\right)\right) \quad \text { for any } x \in \mathcal{B}_{\mu}\left(y_{0}\right) \text { with } d\left(x, \partial \mathcal{B}_{\mu}\left(y_{0}\right)\right) \leq \frac{R_{0}}{2} \text {. }
$$

Using (2.2), Claim 1 follows.

Next, let $e_{0}=y_{0} / \rho\left(y_{0}\right)$ and fix $\sigma<\mu$ so small that $\mathcal{B}_{\sigma}\left(\sigma e_{0}\right) \subset\left\{\mu^{\prime}<\rho\left(x-y_{0}\right)<\mu\right\} \cap \mathcal{B}_{1+\delta}\left(y_{1}\right)$. For $r \in[\sigma-v, \sigma+v]$ and small $v<\sigma$, let us define

$$
\underline{u}_{i}^{\varepsilon}:=\inf _{\partial \mathcal{B}_{r}\left(\sigma e_{0}\right)} u_{i}^{\varepsilon} \quad \text { and } \quad \underline{u}_{i}:=\inf _{\partial \mathcal{B}_{r}\left(\sigma e_{0}\right)} u_{i} .
$$

Since for $r \in[\sigma, \sigma+v], \partial \mathcal{B}_{r}\left(\sigma e_{0}\right) \cap\left(S\left(u_{i}\right)\right)^{c} \neq \emptyset$ and $u_{i} \equiv 0$ on $\left(S\left(u_{i}\right)\right)^{c}$, we have

$$
\underline{u}_{i}=0 \text { for } r \in[\sigma, \sigma+v] \text {. }
$$

By Claim 1, we know that in $B_{\sigma}\left(\sigma e_{0}\right)$ we have

$$
\begin{aligned}
u_{i}(x) & \geq C_{1} d_{\rho}\left(x, \partial \mathcal{B}_{\mu}\left(y_{0}\right)\right) \\
& \geq C_{1} d_{\rho}\left(x, \partial \mathcal{B}_{\sigma}\left(\sigma e_{0}\right)\right) \\
& =C_{1}\left(\sigma-\rho\left(x-\sigma e_{0}\right)\right) .
\end{aligned}
$$

We deduce that for $r \in[\sigma-v, \sigma]$

$$
\underline{u}_{i}=\inf _{\partial \mathcal{B}_{r}\left(\sigma e_{0}\right)} u_{i} \geq \inf _{\partial \mathcal{B}_{r}\left(\sigma e_{0}\right)} C_{1}\left(\sigma-\rho\left(x-\sigma e_{0}\right)\right)=C_{1}(\sigma-r) .
$$

From the previous inequality and (7.3), we infer that

$$
\underline{u}_{i} \geq C_{1}(\sigma-r)^{+}, \quad r \in[\sigma-v, \sigma+v] .
$$

Next, for $j \neq i, r \in[\sigma-v, \sigma+v]$, let us define

$$
\bar{u}_{j}^{\varepsilon}:=\sup _{\partial \mathcal{B}_{1+r}\left(\sigma e_{0}\right)} u_{j}^{\varepsilon} \quad \text { and } \quad \bar{u}_{j}:=\sup _{\partial \mathcal{B}_{1+r}\left(\sigma e_{0}\right)} u_{j} .
$$


The functions $\underline{u}_{i}^{\varepsilon}$ and $\bar{u}_{j}^{\varepsilon}$ are respectively solutions of

$$
\begin{array}{r}
\Delta_{r} \underline{u}_{i}^{\varepsilon} \leq \frac{1}{\varepsilon^{2}} \underline{u}_{i}^{\varepsilon} \sum_{i \neq j} \sup _{\mathcal{B}_{1}\left(\underline{z}_{r}^{i}\right)} u_{j}^{\varepsilon} \\
\Delta_{r} \bar{u}_{j}^{\varepsilon} \geq \frac{1}{\varepsilon^{2}} \bar{u}_{j}^{\varepsilon} \sup _{\mathcal{B}_{1}\left(\bar{z}_{r}^{j}\right)} u_{i}^{\varepsilon}
\end{array}
$$

where

$$
\Delta_{r} u=u_{r r}+\frac{(n-1)}{r} u_{r}=\frac{1}{r^{n-1}} \frac{\partial}{\partial r}\left(r^{n-1} \frac{\partial u}{\partial r}\right)
$$

and $\underline{z}_{r}^{i}$ and $\bar{z}_{r}^{j}$ are respectively the points where the infimum of $u_{i}^{\varepsilon}$ on $\partial \mathcal{B}_{r}\left(\sigma e_{0}\right)$ and the supremum of $u_{j}^{\varepsilon}$ on $\partial \mathcal{B}_{1+r}\left(\sigma e_{0}\right)$ are attained. Note that in spherical coordinates

$$
\Delta u=\Delta_{r} u+\Delta_{\theta} u
$$

and that if we are on a point where $u$ attains a minimum value in the $\theta$ for a fixed $r$ then $\Delta_{\theta} u \geq 0$ and the opposite inequality holds if we are on a maximum point. We also remark that

$$
\bar{y}_{r}^{j}:=\sigma e_{0}+\frac{r}{r+1}\left(\bar{z}_{r}^{j}-\sigma e_{0}\right) \in \partial \mathcal{B}_{r}\left(\sigma e_{0}\right) \cap \partial \mathcal{B}_{1}\left(\bar{z}_{r}^{j}\right)
$$

therefore

$$
\sup _{\mathcal{B}_{1}\left(\bar{z}_{r}^{j}\right)} u_{i}^{\varepsilon} \geq u_{i}^{\varepsilon}\left(\bar{y}_{r}^{j}\right) \geq \underline{u}_{i}^{\varepsilon} .
$$

Moreover, since $\mathcal{B}_{1}\left(\underline{z}_{r}^{i}\right) \subset \mathcal{B}_{1+r}\left(\sigma e_{0}\right)$ and $u_{j}^{\varepsilon}$ is a subharmonic function, we have

$$
\begin{aligned}
\sup _{\mathcal{B}_{1}\left(\underline{z}_{r}^{i}\right)} u_{j}^{\varepsilon} & \leq \sup _{\mathcal{B}_{1+r}\left(\sigma e_{0}\right)} u_{j}^{\varepsilon} \\
& =\sup _{\partial \mathcal{B}_{1+r}\left(\sigma e_{0}\right)} u_{j}^{\varepsilon} \\
& =\bar{u}_{j}^{\varepsilon} .
\end{aligned}
$$

From (7.5), 7.6 and (7.7), we conclude that

$$
\Delta_{r} \underline{u}_{i}^{\varepsilon} \leq \Delta_{r}\left(\sum_{j \neq i} \bar{u}_{j}^{\varepsilon}\right) .
$$

In other words, for any $\phi \in C_{c}^{\infty}(\sigma-v, \sigma+v), \phi \geq 0$, we have

$$
\int_{\sigma-v}^{\sigma+v} \underline{u}_{i}^{\varepsilon} \frac{\partial}{\partial r}\left(r^{n-1} \frac{\partial}{\partial r}\left(\frac{1}{r^{n-1}} \phi\right)\right) d r \leq \int_{\sigma-v}^{\sigma+v} \sum_{j \neq i} \bar{u}_{j}^{\varepsilon} \frac{\partial}{\partial r}\left(r^{n-1} \frac{\partial}{\partial r}\left(\frac{1}{r^{n-1}} \phi\right)\right) d r .
$$


Passing to the limit as $\varepsilon \rightarrow 0$ along a uniformly converging subsequence, we get

$$
\int_{\sigma-v}^{\sigma+v} \underline{u}_{i} \frac{\partial}{\partial r}\left(r^{n-1} \frac{\partial}{\partial r}\left(\frac{1}{r^{n-1}} \phi\right)\right) d r \leq \int_{\sigma-v}^{\sigma+v} \sum_{j \neq i} \bar{u}_{j} \frac{\partial}{\partial r}\left(r^{n-1} \frac{\partial}{\partial r}\left(\frac{1}{r^{n-1}} \phi\right)\right) d r .
$$

The linear growth of $u_{i}$ away from the free boundary given by $(7.3)$ and $(7.4)$, implies that $\Delta_{r} \underline{u}_{i}$ develops a Dirac mass at $r=\sigma$ and

$$
\int_{\sigma-v}^{\sigma+v} \underline{u}_{i} \frac{\partial}{\partial r}\left(r^{n-1} \frac{\partial}{\partial r}\left(\frac{1}{r^{n-1}} \phi\right)\right) d r>0
$$

for $v$ small enough. Hence, $\Delta_{r}\left(\sum_{j \neq i} \bar{u}_{j}\right)$ is a positive measure in $(\sigma-v, \sigma+v)$ and therefore there exists $j \neq i$ such that $u_{j}$ cannot be identically equal to zero in the ball $\mathcal{B}_{1+\sigma}\left(\sigma e_{0}\right)$. Since $\sigma$ small is arbitrary, the result follows.

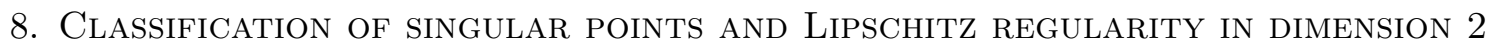

In this section we study singular points in dimension 2. We will always assume 2.8 with $p=1$ in 2.5 . From the results of the previous sections we know that the solutions $u_{1}^{\varepsilon}, \ldots, u_{K}^{\varepsilon}$ of system (2.4), through a subsequence, converge as $\varepsilon \rightarrow 0$ to functions $u_{1}, \ldots, u_{K}$ which are locally Lipschitz continuous in $\Omega$ and harmonic inside their support. For $i=1, \ldots, K$, let us denote the interior of the support of $u_{i}$ by $S_{i}$ as in (6.1) and the union of the interior of the supports of all the other functions by

$$
C_{i}:=\cup_{j \neq i} S_{j}
$$

Since the sets $S_{i}$ are disjoint we have $\partial C_{i}=\cup_{j \neq i} \partial S_{j}$. From Theorem 7.1 we know that $S_{i}$ and $C_{i}$ are at $\rho$-distance 1 , therefore for any point $x \in \partial S_{i}$ there is a point $y \in \partial C_{i}$ such that $\rho(x-y)=1$. We say that $x$ realizes at $y$ the distance from $C_{i}$.

Definition. A point $x \in \partial S_{i}$ is a singular point if it realizes the distance from $C_{i}$ to at least two points in $\partial C_{i}$. We say that $x \in \partial S_{i}$ is a regular point if it is not singular.

Geometrically, we can describe regular and singular points as follows. Let $x \in \partial S_{i}$ be a singular point and $y_{1}, y_{2} \in \partial C_{i}$ points where $x$ realizes the distance from $C_{i}$. Then the balls 


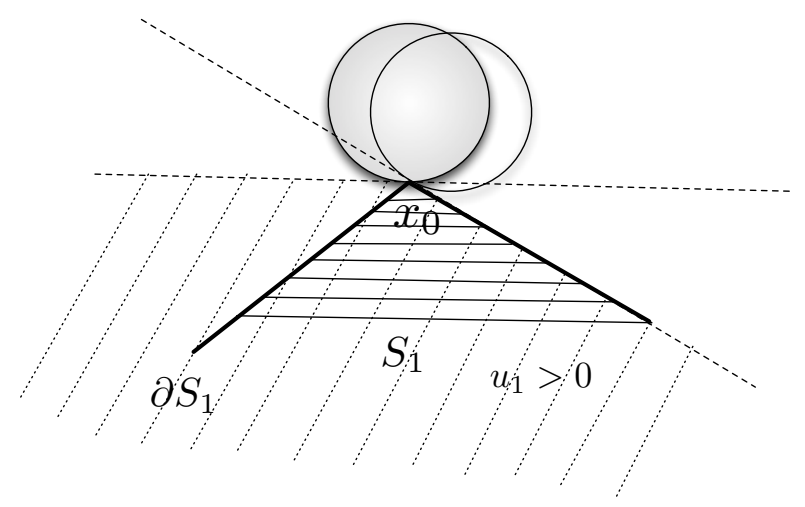

Figure 2. Asymptotic cone at $x_{0}$

$\mathcal{B}_{1}\left(y_{1}\right)$ and $\mathcal{B}_{1}\left(y_{2}\right)$ are tangent to $\partial S_{i}$ at $x$. Consider the convex cone determined by the two tangent lines to the two tangent $\rho$-balls $\mathcal{B}_{1}\left(y_{1}\right)$ and $\mathcal{B}_{1}\left(y_{2}\right)$, which does not intersect the two $\rho$-balls. The intersection of all cones generated by all $\rho$-balls of radius 1 , tangent at $x$ and with center in $C_{i}$ defines a convex asymptotic cone centered at $x$, see Figure 2, If $x \in \partial S_{i}$ is a regular point, then there is only one point $y \in \partial C_{i}$ where $x$ realizes the distance from $C_{i}$. In this case, the two tangent balls coincide and therefore, by definition the asymptotic cone at $x \in \partial S_{i}$ is an half-plane. We will show that at regular points $\partial S_{i}$ is the graph of a differentiable function. If $\theta \in[0, \pi]$ is the opening of the cone at $x$, we say that $S_{i}$ has an angle $\theta$ at $x$. Regular points correspond to $\theta=\pi$. When $\theta=0$ the tangent cone is actually a semi-line and $S_{i}$ has a cusp at $x$. We will show, later on in this section, that, assuming additional hypothesis on the boundary data and the domain $\Omega$, the case $\theta=0$ never occurs and therefore the free boundaries are Lipschitz curves of the plane.

Lemma 8.1. Let $\mathcal{C}=\left\{\left(x_{1}, x_{2}\right): x_{2} \geq \alpha\left|x_{1}\right|\right\}, \alpha \geq 0$, be the asymptotic cone of $S_{i}$ at $0 \in \partial S_{i}$. Then there exist $y_{1}, y_{2} \in \partial C_{i}$ such that the balls $\mathcal{B}_{1}\left(y_{1}\right)$ and $\mathcal{B}_{1}\left(y_{2}\right)$ are tangent respectively to the lines $x_{2}= \pm \alpha x_{1}$ at 0 .

Proof. Let $y_{1}, y_{2} \in \mathcal{B}_{1}(0)$ be such that $x_{2}=\alpha x_{1}$ is a tangent line to $\mathcal{B}_{1}\left(y_{1}\right)$ at 0 and $x_{2}=-\alpha x_{1}$ is a tangent line to $\mathcal{B}_{1}\left(y_{2}\right)$ at 0 . Suppose by contradiction that $y_{1}, y_{2} \notin \partial C_{i}$. Then, any $y \in C_{i}$ such 
that $\rho(y-0)=1$ must lie in the smaller arc in $\partial \mathcal{B}_{1}(0)$ between $y_{1}$ and $y_{2}$. Moreover, there exists $\delta>0$ such that all $\rho$-balls $\mathcal{B}_{1}(y)$ have at most as tangent lines at 0 the lines $x_{2}= \pm(\alpha-\delta) x_{1}$. Then the asymptotic cone at 0 must contain the cone $\left\{\left(x_{1}, x_{2}\right): x_{2} \geq(\alpha-\delta)\left|x_{1}\right|\right\}$, which is not possible.

Lemma 8.2. Assume that $S_{i}$ has an angle $\theta \in(0, \pi]$ at $x_{0} \in \partial S_{i}$. Then, there exists a neighborhood $U$ of $x_{0}$, a system of coordinates $\left(x_{1}, x_{2}\right)$ and a locally Lipschitz function $\psi$ : $(-r, r) \rightarrow \mathbb{R}$, for some $r>0$, such that in the system of coordinates $\left(x_{1}, x_{2}\right)$, we have that $x_{0}=(0,0)$ and

$$
\partial S_{i} \cap U=\left\{\left(x_{1}, \psi\left(x_{1}\right): x_{1} \in(-r, r)\right\} .\right.
$$

If in addition $\theta=\pi$, then $\varphi$ is differentiable at 0 .

Proof. Let $\mathcal{C}$ be the convex asymptotic cone of $S_{i}$ at $x_{0}$. Let us fix a system of coordinates $\left(x_{1}, x_{2}\right)$ such that the $x_{2}$ axis coincides with the axis of the cone and is oriented such that the cone is above the $x_{1}$ axis. Then we have that $x_{0}=(0,0)$ and $\mathcal{C}=\left\{\left(x_{1}, x_{2}\right): x_{2} \geq \alpha\left|x_{1}\right|\right\}$ with $\alpha=\tan \left(\frac{\pi-\theta}{2}\right)$. To prove that in this system of coordinates, $\partial S_{i}$ is the graph of a function in a small neighborhood of $x_{0}$, it suffices to show that there exists a small $r>0$ such that, for any $|t|<r$, the vertical line $\left\{x_{1}=t\right\}$, intersects $\partial S_{i} \cap B_{r}(0)$ at only one point. Suppose by contradiction that there exists a sequence $\left(t_{n}\right)$ such that $t_{n} \rightarrow 0$ as $n \rightarrow+\infty$, and the line $\left\{x_{1}=t_{n}\right\}$ intersects $\partial S_{i} \cap B_{r}(0)$ at two distinct points $\left(t_{n}, a_{n}\right)$ and $\left(t_{n}, b_{n}\right)$ with $b_{n}>a_{n}$. Assume, without loss of generality, that $t_{n}>0$ for any $n$. By Lemma 8.1 there exist $y_{1}$, $y_{2} \in \partial C_{i}$ that realize the distance from 0 , and such that $\mathcal{B}_{1}\left(y_{1}\right)$ is tangent to the line $\left\{\left(x_{1}, x_{2}\right)\right.$ : $\left.x_{2}=\alpha x_{1}\right\}$ at 0 and $\mathcal{B}_{1}\left(y_{2}\right)$ is tangent to $\left\{\left(x_{1}, x_{2}\right): x_{2}=-\alpha x_{1}\right\}$ also at 0 . For instance, in the particular case of the Euclidean norm, we would have $y_{1}=\left(\sqrt{\frac{1}{1+\alpha^{2}}},-\alpha \sqrt{\frac{1}{1+\alpha^{2}}}\right)$ and $y_{2}=\left(-\sqrt{\frac{1}{1+\alpha^{2}}},-\alpha \sqrt{\frac{1}{1+\alpha^{2}}}\right)$. In general, what we can say is that the $x_{2}$ coordinate of $y_{1}$ and $y_{2}$ is a negative value $-c$. We have that $\mathcal{B}_{1}\left(y_{1}\right) \cap \mathcal{B}_{1}\left(y_{2}\right) \neq \emptyset$, since $\theta>0$. Moreover, $S_{i} \cap\left(\mathcal{B}_{1}\left(y_{1}\right) \cup \mathcal{B}_{1}\left(y_{2}\right)\right)=\emptyset$. Then, both points $\left(t_{n}, a_{n}\right)$ and $\left(t_{n}, b_{n}\right)$ must be above $\mathcal{B}_{1}\left(y_{1}\right) \cup \mathcal{B}_{1}\left(y_{2}\right)$ 
for $n$ large enough. Next, let $y_{n}^{a}, y_{n}^{b} \in \partial C_{i}$ be points where $\left(t_{n}, a_{n}\right)$ and $\left(t_{n}, b_{n}\right)$, respectively, realize the distance from $C_{i}$. Then the $\rho$-balls $\mathcal{B}_{1}\left(y_{n}^{a}\right)$ and $\mathcal{B}_{1}\left(y_{n}^{b}\right)$ are exterior tangent balls to $\partial S_{i}$ at $\left(t_{n}, a_{n}\right)$ and $\left(t_{n}, b_{n}\right)$, respectively. Recall that the $\rho$-distance between the points $\left(t_{n}, a_{n}\right)$ and $\left(t_{n}, b_{n}\right)$ converges to 0 as $n \rightarrow+\infty$, and so, the point $y_{n}^{a}$ has to belong to the lower half $\rho$-ball $\partial \mathcal{B}_{1}\left(t_{n}, a_{n}\right) \cap\left\{x_{2}<a_{n}\right\}$ for $n$ large enough. Indeed, if not the tangent $\rho$-ball $\mathcal{B}_{1}\left(y_{n}^{a}\right)$ would contain $\left(t_{n}, b_{n}\right)$ for $n$ large enough. Similarly, $y_{n}^{b}$ has to belong to the upper half $\rho$-ball $\partial \mathcal{B}_{1}\left(t_{n}, b_{n}\right) \cap\left\{x_{2}>b_{n}\right\}$ for $n$ large enough. This implies that the tangent $\rho$-ball $\mathcal{B}_{1}\left(y_{n}^{b}\right)$ will converge to a tangent ball to $S_{i}$ at $0, \mathcal{B}_{1}\left(y^{b}\right)$, with $y^{b} \in\left\{x_{2} \geq 0\right\}$. On the other hand, by the definition of the asymptotic cones, all the centers of the tangent balls at 0 must belong to the set $\partial \mathcal{B}_{1}(0) \cap\left\{x_{2} \leq-c\right\}$, where $-c<0$ is the $x_{2}$ coordinate of the points $y_{1}, y_{2}$ defined above. Therefore, we have reached a contradiction. We infer that there exists $r>0$ such that $\partial S_{i}$ is the graph of a function $\psi:(-r, r) \rightarrow \mathbb{R}$. Since $\partial S_{i}$ is a closed set, $\psi$ is continuous.

Let us prove that $\psi$ is Lipschitz continuous at 0 . If $\mathcal{C}=\left\{x_{2} \geq \alpha\left|x_{1}\right|\right\}$ is the tangent cone of $S_{i}$ at $x_{0}$ in the system of coordinates $\left(x_{1}, x_{2}\right)$, then for $r>0$ small enough we have

$$
\left\{x_{2} \geq 2 \alpha\left|x_{1}\right|\right\} \subset S_{i} \cap B_{r}(0) \subset\left\{x_{2} \geq \frac{\alpha}{2}\left|x_{1}\right|\right\}
$$

that is, for $\left|x_{1}\right|<r$,

$$
\frac{\alpha}{2}\left|x_{1}\right| \leq \psi\left(x_{1}\right)=\psi\left(x_{1}\right)-\psi(0) \leq 2 \alpha\left|x_{1}\right|
$$

Therefore, $\psi$ is Lipschitz at 0.

Next, assume that $\theta=\pi$. Then, we have that $y_{1}=y_{2}$, and $x_{0}$ is a regular point. Therefore, $\mathcal{B}_{1}\left(y_{1}\right) \subset\left\{x_{2}<0\right\}$ is the unique tangent ball to the graph of $\psi$ at $x_{0}=(0,0)$. Moreover, the tangent cone is the half plane $\left\{x_{2} \geq 0\right\}$. Let us show that $\psi$ is differentiable at 0 . Assume by contradiction that there exists a sequence of positive points $\left(x_{1}^{n}\right) \in(-r, r)$ such that $x_{1}^{n} \rightarrow 0$ as $n \rightarrow+\infty$ and

$$
\lim _{n \rightarrow+\infty} \frac{\psi\left(x_{1}^{n}\right)}{x_{1}^{n}}=\beta \neq 0 .
$$


Since there exists a tangent ball from below to the graph of $\psi$ at 0 contained in $\left\{x_{2}<0\right\}$, we must have $\beta>0$. For any point $\left(x_{1}^{n}, \psi\left(x_{1}^{n}\right)\right) \in \partial S_{i}$ there exists a point $y_{n} \in \partial C_{i}$ such that $\mathcal{B}_{1}\left(y_{n}\right)$ is tangent to $S_{i}$ at $\left(x_{1}^{n}, \psi\left(x_{1}^{n}\right)\right)$. Let $y_{2} \in \partial C_{i}$ be the limit of a converging subsequence of $\left(y_{n}\right)$. Then the $\rho$-ball $\mathcal{B}_{1}\left(y_{2}\right)$ is an exterior tangent ball at $S_{i}$ at 0 . Equation 8.2 gives $\psi\left(x_{1}^{n}\right) \geq \frac{\beta}{2} x_{1}^{n}$ for $n$ large enough, i.e., the points $\left(x_{1}^{n}, \psi\left(x_{1}^{n}\right)\right)$ of the free boundary are above the line $\left\{x_{2}=\beta / 2\left|x_{1}\right|\right\}$. This implies that $y_{1} \neq y_{2}$, that is the limit $\rho$-ball $\mathcal{B}_{1}\left(y_{2}\right)$ must be different from $\mathcal{B}_{1}\left(y_{1}\right)$. This is in contradiction with the fact that $x_{0}$ is a regular point. Therefore we must have

$$
\lim _{x_{1} \rightarrow 0^{+}} \frac{\psi\left(x_{1}\right)}{x_{1}}=0
$$

Similarly, one can prove that

$$
\lim _{x_{1} \rightarrow 0^{-}} \frac{\psi\left(x_{1}\right)}{x_{1}}=0
$$

We conclude that $\psi$ is differentiable at 0 and $\psi^{\prime}(0)=0$.

Lemma 8.3. Assume that there exists an open set $U$ of $\mathbb{R}^{2}$ such that any point of $U \cap \partial S_{i}$ is regular. Then $U \cap \partial S_{i}$ is a $C^{1}$-curve of the plane.

Proof. Let $y_{0} \in \partial S_{i} \cap U$. By Lemma 8.2, there exists a differentiable function $\psi$ and a small $r>0$, such that, in the system of coordinates $\left(x_{1}, x_{2}\right)$ centered at $y_{0}$ and with the $x_{2}$ axis in the direction of the inner normal of $\partial S_{i}$ at $y_{0}, \partial S_{i} \cap B_{r}\left(y_{0}\right)$ is the graph of $\psi$. Moreover, in this system of coordinates, $\psi\left(y_{0}\right)=\psi^{\prime}\left(y_{0}\right)=0$. By Corollary 6.2, there exists a tangent ball from below, with uniform radius, at any point of the graph of $\psi$. This implies that for any $\left|x_{1}^{0}\right|<r$, there exists a $C^{2}$ function $\varphi_{x_{1}^{0}}$ tangent from below to the graph of $\psi$ at $x_{1}^{0}$ and such that $\left|\varphi_{x_{1}^{0}}^{\prime \prime}\right| \leq C$, for some $C>0$ independent of $x_{1}^{0}$. Therefore we have, for any $\left|x_{1}\right|<r$,

$$
\begin{aligned}
\psi\left(x_{1}\right) & \geq \varphi_{x_{1}^{0}}\left(x_{1}\right) \geq \varphi_{x_{1}^{0}}\left(x_{1}^{0}\right)+\varphi_{x_{1}^{0}}^{\prime}\left(x_{1}^{0}\right)\left(x_{1}-x_{1}^{0}\right)-C\left|x_{1}-x_{1}^{0}\right|^{2} \\
& =\psi\left(x_{1}^{0}\right)+\psi^{\prime}\left(x_{1}^{0}\right)\left(x_{1}-x_{1}^{0}\right)-C\left|x_{1}-x_{1}^{0}\right|^{2} .
\end{aligned}
$$


Now, let us show that $\psi$ is of class $C^{1}$. Fix a point $x_{1}^{0}$ and consider a sequence $\left(x_{1}^{l}\right)$ converging to $x_{1}^{0}$ as $l \rightarrow+\infty$. Let $p$ be the limit of a convergent subsequence of $\left(\psi^{\prime}\left(x_{1}^{l}\right)\right)$. Passing to the limit in $l$ the inequality,

$$
\psi\left(x_{1}\right) \geq \psi\left(x_{1}^{l}\right)+\psi^{\prime}\left(x_{1}^{l}\right)\left(x_{1}-x_{1}^{l}\right)-C\left|x_{1}-x_{1}^{l}\right|^{2},
$$

we get

$$
\psi\left(x_{1}\right) \geq \psi\left(x_{1}^{0}\right)+p\left(x_{1}-x_{1}^{0}\right)-C\left|x_{1}-x_{1}^{0}\right|^{2},
$$

for any $\left|x_{1}\right|<r$. Since $\psi$ is differentiable at $x_{1}^{0}$, we must have $p=\psi^{\prime}\left(x_{1}^{0}\right)$.

Lemma 8.4. Assume that the supports of the boundary data, $f_{i}$ 's, on $(\partial \Omega)_{1}$ have a finite number of connected components. Then the sets $S_{i}$ 's have a finite number of connected components.

Proof. Consider all the connected components of $S_{i}, S_{i}^{j}, i=1, \ldots, K$ and $j=1,2,3, \ldots$. Remark that for any $i$ and $j$

$$
\partial S_{i}^{j} \cap\left\{x \in(\partial \Omega)_{1}: f_{i}(x)>0\right\} \neq \emptyset .
$$

Indeed, if not we would have $u_{i}=0$ on $\partial S_{i}^{j}$ and $\Delta u_{i} \geq 0$ in $S_{i}^{j}$. The maximum principle then would imply $u_{i} \equiv 0$ in $S_{i}^{j}$, which is not possible. Moreover, by continuity, $\partial S_{i}^{j}$ must contain one connected component of the set $\left\{x \in(\partial \Omega)_{1}: f_{i}(x)>0\right\}$. For this reasons we say that the components of $S_{i}$ reach the boundary of $\Omega$. This implies that the connected components of $S_{i}$ are finite.

8.1. Properties of singular points. We start by proving three lemmas that will allow to estimate the growth of the solutions near the singular points. The first lemma claims that positive functions which are superharmonic (subharmonic) in a cone and vanish on its boundary, have at least (at most) linear growth away from the boundary of the cone far from the vertex, with a slope that degenerate in a Hölder fashion approaching the vertex. The power just depends on the opening of the cone. The second and third lemmas generalize these estimates to domains which are sets of points at $\rho$-distance greater than 1 from a closed bounded set. Then we prove 
that the set of singularities is a set of isolated points and we give a characterization. For the set $S_{i}$ which has finite perimeter, we denote by $\partial^{*} S_{i}$ the reduced boundary, that is the set of points whose blow-ups converge to half-planes and the essential boundary, $\partial_{*} S_{i}$, are all points except points of Lebesgue density zero and one. Moreover, $\mathcal{H}^{1}\left(\partial_{*} S_{i} \backslash \partial^{*} S_{i}\right)=0$. For more details see [1,22].

Lemma 8.5. Let $v$ be a nonnegative Lipschitz function defined on $B_{1} \subset \mathbb{R}^{n}$, such that $\Delta v$ is locally a Radon measure on $B_{1}$ and such that $v$ smooth on $S=\{v>0\}$. Assume that $S$ is a set of finite perimeter. Then, for every smooth $\phi$ with compact support contained in $B_{1}$

$$
\int_{B_{1}} \Delta v \phi=\int_{S} \Delta v \phi d x-\int_{\partial^{*} S} \frac{\partial v}{\partial \nu_{S}} \phi d \mathcal{H}^{n-1}
$$

where $\nu_{S}$ is the measure-theoretic outward unit normal and $\partial^{*} S$ is the reduced boundary.

Proof. As a distribution and integrating by parts

$$
\int_{B_{1}} \Delta v \phi=\int_{S} v \Delta \phi d x=\int_{S} \operatorname{div}(v \nabla \phi)-\operatorname{div}(\nabla v \phi)+\Delta v \phi d x
$$

Applying the generalized Gauss-Green theorem (see [7, and also [1,22 for more details) we obtain the result.

Lemma 8.6. Let $\theta_{0} \in(0, \pi]$. Let $\mathcal{C}$ be the cone defined in polar coordinates by

$$
\mathcal{C}=\left\{(\varrho, \theta) \mid \varrho \in[0,+\infty), 0 \leq \theta \leq \theta_{0}\right\}
$$

Let $u_{1}$ and $u_{2}$ be respectively a superharmonic and subharmonic positive function in the interior of $\mathcal{C} \cap B_{2 r_{0}}$, such that $u_{1} \geq u_{2}=0$ on $\partial \mathcal{C} \cap B_{2 r_{0}}$. Then for any $r<r_{0} / 3$ there exist $R=R\left(\theta_{0}, r\right)$, and constants $c, C>0$ depending on respectively $\left(\theta_{0}, u_{1}, r_{0}\right)$ and $\left(\theta_{0}, u_{2}, r_{0}\right)$, but independent of $r$, such that for any $x \in[r, 3 r] \times[0, R]$ we have

(1) $u_{1}(x) \geq c r^{\alpha} d(x, \partial \mathcal{C})$

(2) $u_{2}(x) \leq C r^{\alpha} d(x, \partial \mathcal{C})$ 
where $\alpha$ is given by

$$
1+\alpha=\frac{\pi}{\theta_{0}}
$$

Proof. Let us introduce the function

$$
v(\varrho, \theta):=\varrho^{1+\alpha} \sin ((1+\alpha) \theta)
$$

Notice that $v$ is harmonic in the interior of $\mathcal{C}$, since it is the imaginary part of the function $z^{1+\alpha}$, where $z=x+i y$, which is holomorphic in the set $\mathbb{C} \backslash(-\infty, 0]$. Moreover $v$ is positive inside $\mathcal{C}$ and vanishes on its boundary. By a barrier argument, $u_{1}$ has at least linear growth away from the boundary of $\mathcal{C}$, meaning for $\rho \in\left[r_{0} / 2,3 r_{0} / 2\right]$ (far from the vertex and from $\partial B_{2 r_{0}}$ )

$$
u_{1}(x) \geq k d(x, \partial \mathcal{C})
$$

for $k=c_{0} \min \underset{d(x, \partial \mathcal{C}) \geq s_{0}}{x \in \mathcal{C}} u_{1}$, and for $x \in\left\{x \in \mathcal{C}: r_{0} / 2<|x|<3 r_{0} / 2, d(x, \partial \mathcal{C}) \leq s_{0}\right\}$ where $c_{0}$ and $s_{0}$ depend on $r_{0}$ and $\theta_{0}$. Therefore, we can find a constant $c>0$ depending on $u_{1}, r_{0}$ and $\theta_{0}$, such that

$$
u_{1} \geq c v \quad \text { on } \mathcal{C} \cap \partial B_{r_{0}}
$$

Since in addition $u_{1} \geq c v=0$ on $\partial C \cap B_{r_{0}}$, the comparison principle implies

$$
u_{1} \geq c v \quad \text { in } \mathcal{C} \cap B_{r_{0}}
$$

Since $v$ is increasing in the radial direction and if we are near $\partial \mathcal{C}$ it is also increasing in the $\theta$ direction, for $r \leq|x| \leq 3 r$, with $r$ such that $r \leq \frac{r_{0}}{3}$ and $d(x, \mathcal{C}) \leq R$ with $R=r \min \left\{1, \tan \left(\frac{\theta_{0}}{2}\right)\right\}$,

$$
u_{1}(x) \geq c v(x) \geq C r^{\alpha} d(x, \partial \mathcal{C})
$$

and (a) follows.

To prove (b) similarly, we have

$$
u_{2} \leq C v \quad \text { in } \mathcal{C} \cap B_{r_{0}}
$$


where $C$ depends on $\left(\theta_{0}, u_{2}, r_{0}\right)$ but it is independent of $r$. In particular, for $r \leq|x| \leq 3 r$ and $d(x, \mathcal{C}) \leq \frac{R}{2}$

$$
u_{2}(x) \leq C v(x) \leq \tilde{C} r^{\alpha} d(x, \partial \mathcal{C})
$$

Lemma 8.7. Let $\Omega$ be an open set, $C$ be a closed subset of $\Omega$ and $S=\left\{x \in \Omega \mid d_{\rho}(x, C) \geq 1\right\}$. Let $S_{1}$ be a connected component of $S$. Assume that $\partial S_{1}=\Gamma_{1} \cup \Gamma_{2}$, with $\Gamma_{1} \cap \Gamma_{2}=\{0\}$ and $S_{1}$ has an angle $\theta_{0} \in(0, \pi]$ at $0 \in \partial S_{1}$. Let $u_{1}$ be a superharmonic positive function in $S_{1} \cap B_{2 r_{0}}(0)$, with $u_{1}=0$ on $\partial S_{1} \cap B_{2 r_{0}}(0)$. Then, there exists a sequence $\left(x_{h}\right) \subset \Gamma_{1}$ of regular points convergent to zero, $x_{h} \rightarrow 0$ as $h \rightarrow 0$, and there exist balls $B_{R_{h}}\left(z_{h}\right) \subset S_{1}$ tangent to $\partial S_{1}$ at $x_{h}$, where $R_{h} \geq c\left|x_{h}\right|$, such that

$$
u_{1}(x) \geq c R_{h}^{\alpha_{\delta}} d\left(x, \partial B_{R_{h}}\left(z_{h}\right)\right) \quad \text { for any } \quad x \in B_{R_{h}}\left(z_{h}\right) \backslash B_{\frac{R_{h}}{4}}\left(z_{h}\right)
$$

where $\alpha_{\delta}$ is given by

$$
1+\alpha_{\delta}=\frac{\pi}{\theta_{0}-\delta}
$$

Proof. Since $\theta_{0} \in(0, \pi]$ for any $0<\delta<\theta_{0}$, there exist $r_{\delta}>0$ and a cone $\mathcal{C}_{\delta}^{1}$ centered at 0 with opening $\theta_{0}-\delta$ such that

$$
\mathcal{C}_{\delta}^{1} \cap B_{r_{\delta}}(0) \subset S_{1} \cap B_{r_{\delta}}(0)
$$

Take a sequence of points $t_{h} \in \partial \mathcal{C}_{\delta}^{1} \cap B_{r_{\delta}}(0)$ converging to 0 as $h \rightarrow 0$. Let

$$
r_{h}:=d\left(t_{h}, 0\right) \quad \text { and } \quad R_{h}:=r_{h} \min \left\{1, \tan \left(\frac{\theta_{0}-\delta}{2}\right)\right\} .
$$

Then, for $h$ small enough, there exist balls $B_{R_{h}}\left(s_{h}\right) \subset \mathcal{C}_{\delta}^{1} \cap B_{r_{\delta}}(0)$ such that $t_{h} \in \partial B_{R_{h}}\left(s_{h}\right)$. Consider a system of polar coordinates $(\varrho, \theta)$ centered at 0 . Moving the balls $B_{R_{h}}\left(s_{h}\right)$ along the $\theta$ direction until it touches $\Gamma_{1}$, we can find a sequence of regular points $x_{h}$ in that region, such that $d\left(x_{h}, 0\right) \leq c r_{h}$ and balls $B_{R_{h}}\left(z_{h}\right) \subset S_{1} \cap B_{r_{\delta}}(0)$ such that $x_{h} \in \partial B_{R_{h}}\left(z_{h}\right)$. Observe that the center of the ball, $z_{h}$, remains inside the cone $\mathcal{C}_{\delta}^{1}$, that is, for $h$ and $\delta$ small enough, we 
have that $z_{h} \in \mathcal{C}_{\delta}^{1}$ and $d\left(z_{h}, \partial \mathcal{C}_{\delta}^{1}\right) \geq \frac{R_{h}}{2}$. Let us introduce the barrier function

$$
\phi(x):=\frac{m}{\log 4} \log \left(\frac{R_{h}}{\left|x-z_{h}\right|}\right), \quad \text { where } \quad m=\inf _{\partial B_{\frac{R_{h}}{4}}\left(z_{h}\right)} u_{1} .
$$

Then $\phi$ satisfies

$$
\begin{cases}\Delta \phi=0 & \text { in } B_{R_{h}}\left(z_{h}\right) \backslash B_{\frac{R_{h}}{4}}\left(z_{h}\right) \\ \phi=0 & \text { on } \partial B_{R_{h}}\left(z_{h}\right) \\ \phi=m & \text { on } \partial B_{\frac{R_{h}}{4}}\left(z_{h}\right) .\end{cases}
$$

Since $u_{1} \geq \phi$ on $\partial B_{R_{h}}\left(z_{h}\right) \cup \partial B_{\frac{R_{h}}{4}}\left(z_{h}\right)$ the comparison principle then implies

$$
u_{1} \geq \phi \quad \text { in } B_{R_{h}}\left(z_{h}\right) \backslash B_{\frac{R_{h}}{4}}\left(z_{h}\right)
$$

If $\nu_{1}$ is the inner normal vector of $B_{R_{h}}\left(z_{h}\right)$, then for $x \in \partial B_{R_{h}}\left(z_{h}\right)$,

$$
\frac{\partial \phi}{\partial \nu_{1}}(x)=\frac{m}{R_{h} \log 4}
$$

and the convexity of $\phi$ in the radial direction gives, for any $x \in B_{R_{h}}\left(z_{h}\right) \backslash B_{\frac{R_{h}}{4}}\left(z_{h}\right)$

$$
u_{1}(x) \geq \frac{m}{R_{h} \log 4} d\left(x, \partial B_{R_{h}}\left(z_{h}\right)\right) .
$$

Let us estimate $m$. Since $d\left(z_{h}, \partial C_{\delta}^{1}\right) \geq \frac{R_{h}}{2}$, we have that $d\left(x, \partial C_{\delta}^{1}\right) \geq \frac{R_{h}}{4}$ for any $x \in B_{\frac{R_{h}}{4}}\left(z_{h}\right)$. As in Lemma 8.6, consider the harmonic function $v(x)$, introduced in (8.3), defined on the cone $\mathcal{C}_{\delta}^{1}\left(\alpha=\alpha_{\delta}\right)$ and the comparison principle result stated in $(8.4)$. Then

$$
m \geq c \min _{\partial B_{\frac{R_{h}}{4}}\left(z_{h}\right)} v \geq \min \left\{v\left(r_{h}-\frac{R_{h}}{4}, \frac{\theta_{0}-\delta}{8}\right), v\left(\frac{3 r_{h}}{4}, \frac{\pi}{16}\right)\right\}=c_{1}\left(\frac{3 r_{h}}{4}\right)^{\alpha_{\delta}+1}
$$

where $c_{1}=c_{1}\left(u_{1}, r_{\delta}, \theta_{0}-\delta\right)$. Then, since $\frac{r_{h}}{R_{h}} \geq 1$ we conclude that for any $x \in B_{R_{h}}\left(z_{h}\right) \backslash B_{\frac{R_{h}}{4}}\left(z_{h}\right)$,

$$
u_{1}(x) \geq c R_{h}^{\alpha_{\delta}} d\left(x, \partial B_{R_{h}}\left(z_{h}\right)\right)
$$

This concludes the proof of the lemma.

Lemma 8.8. Let $\Omega$ be an open set, $C$ be a closed subset of $\Omega$ and $S=\left\{x \in \Omega \mid d_{\rho}(x, C) \geq 1\right\}$. Let $S_{1}$ be a connected component of $S$. Assume that $S_{1}$ has an angle $\theta_{0} \in[0, \pi]$ at $0 \in \partial S_{1}$. Let $u_{2}$ be a subharmonic positive function in $S_{1} \cap B_{2 r_{0}}(0)$, with $u_{2}=0$ on $\partial S_{1} \cap B_{2 r_{0}}(0)$. 
Then, for any $0<\delta<\theta_{0}$, there exists $r_{\delta}>0$ such that for any $r<r_{\delta} / 5$ there exist $R=R\left(\theta_{0}, r\right)$, and a constant $C>0$ depending on $\left(\theta_{0}+\delta, u_{2}, r_{\delta}\right)$, but independent of $r$, such that

$$
u_{2}(x) \leq C r^{\beta_{\delta}} d\left(x, \partial S_{1}\right) \quad \text { for any } \quad x \in\left(B_{3 r}(0) \backslash B_{r}(0)\right) \cap\left\{x \in S_{1}: d\left(x, \partial S_{1}\right) \leq \frac{R}{4}\right\}
$$

where $\beta_{\delta}$ is given by

$$
1+\beta_{\delta}=\frac{\pi}{\theta_{0}+\delta}
$$

Proof. For any $\delta>0$, there exist $r_{\delta}>0$, a cone $\mathcal{C}_{\delta}^{2}$ centered at 0 and with opening $\theta_{0}+\delta$ such that

$$
S_{1} \cap B_{r_{\delta}}(0) \subset \mathcal{C}_{\delta}^{2} \cap B_{r_{\delta}}(0)
$$

Take any $r<r_{\delta}$ and let $y \in \partial S \cap\left(B_{3 r}(0) \backslash B_{r}(0)\right)$ and $r_{y}:=d(y, 0) \in(r, 3 r)$. Since $S$ is at $\rho$-distance 1 from $C$, for any point of the boundary of $S_{1}$ there exists an exterior tangent $\rho$-ball of radius 1 . This implies that for $r$ small enough, there exists $w_{y}$ such that the Euclidian ball $B_{R_{y}}\left(w_{y}\right)$ is contained in the complement of $S$ and $y \in \partial B_{R_{y}}\left(w_{y}\right)$, where $R_{y}$ is defined by

$$
R_{y}=r_{y} \min \left\{1, \tan \left(\frac{\theta_{0}+\delta}{2}\right)\right\} \text {. }
$$

Let us take now as barrier the function

$$
\psi(x):=\frac{M}{\log \frac{3}{2}} \log \left(\frac{\left|w_{y}-x\right|}{R_{y}}\right) \quad \text { with } \quad M=\sup _{\partial B_{\frac{3}{2} R_{y}}\left(w_{y}\right)} u_{2} .
$$

Then $\psi$ satisfies

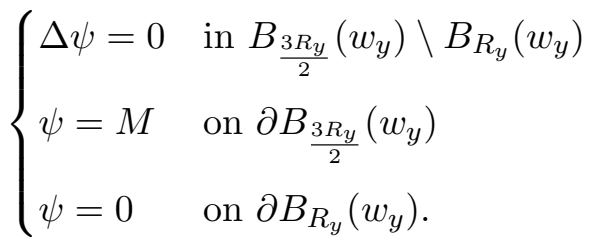

Using the comparison principle with $u_{2}$, the concavity of $\psi$ in the radial direction gives that for any $x \in B_{\frac{3 R_{y}}{2}}\left(w_{y}\right) \backslash B_{R_{y}}\left(w_{y}\right)$

$$
u_{2} \leq \frac{M}{R_{y} \log \left(\frac{3}{2}\right)} d\left(x, \partial B_{R_{y}}\left(w_{y}\right)\right) .
$$


Let us estimate $M$. Consider again a system of polar coordinates $(\varrho, \theta)$ centered at 0 and the harmonic function $v(x)$, introduced in $(8.3)$, defined on the cone $\mathcal{C}_{\delta}^{2}\left(\alpha=\beta_{\delta}\right)$. By definition of $v, R_{y}$, and taking into account 8.5 , for $\delta, r$ small enough,

$$
M \leq C \max _{\partial B_{\frac{3 R_{y}}{2}}\left(w_{y}\right)} v \leq C v\left(4 r_{y}, \frac{\theta_{0}+\delta}{2}\right)=C_{1}\left(4 r_{y}\right)^{\beta_{\delta}+1}=\tilde{C}_{1} r_{y}^{\beta_{\delta}} \frac{R_{y}}{\min \left\{1, \tan \left(\frac{\theta_{0}+\delta}{2}\right)\right\}}
$$

we see that for any $x \in \frac{B_{\frac{3 R_{y}}{2}}}{}\left(w_{y}\right) \backslash B_{R_{y}}\left(w_{y}\right)$ and belonging to the segment $y+s\left(y-w_{y}\right)$, $s \in\left(0, \frac{1}{2}\right)$, we have

$$
u_{2}(x) \leq C M d\left(x, \partial B_{R_{y}}\left(w_{y}\right)\right)=C M d\left(x, \partial S_{1}\right) \leq C r_{y}^{\beta_{\delta}} d\left(x, \partial S_{1}\right)
$$

Letting the tangent ball moving along $\partial S_{1} \cap\left(B_{3 r_{y}}(0) \backslash B_{r_{y}}(0)\right)$, we get (b).

Lemma 8.9. Assume 2.8 with $n=2$ and $p=1$ in 2.5. Assume in addition that the supports on $\partial \Omega$ of the boundary data $f_{i}$ 's have a finite number of connected components. Let $\left(u_{1}^{\varepsilon}, \ldots, u_{K}^{\varepsilon}\right)$ be a viscosity solution of the problem (2.4) and $\left(u_{1}, \ldots, u_{K}\right)$ the limit as $\varepsilon \rightarrow 0$ of a convergent subsequence. The set of singular points of $\Omega$ is a set of isolated points.

Proof. Suppose by contradiction that there exists a sequence of distinct singular points $\left(y_{k}\right)_{k \in \mathbf{N}}$ such that $y_{k} \in \partial S_{j}$ and $y_{k} \rightarrow y \in \Omega$ as $k \rightarrow+\infty$. Since by Lemma 8.4 the connected components of the sets $S_{i}, i=1, \ldots, K$ are finite, we may assume without loss of generality that the points $y_{k}$ belong to the same connected component of $S_{j}$, which we denote by $S_{j}^{1}$. If there exists $\theta_{\max }<\pi$ such that $S_{j}^{1}$ has an angle smaller than $\theta_{\max }$ at $y_{k}$ for any $k$, then, there exists $\bar{k}$ such that starting from $y_{\bar{k}}$, after a finite number of singular points $S_{j}^{1}$ would be an isle and not reach the boundary. Therefore we would have $u_{j}=0$ on $\partial S_{j}^{1}$ and $\Delta u_{j}=0$ in $S_{j}^{1}$, and the maximum principle would imply $u_{j} \equiv 0$ in $S_{j}^{1}$, which is a contradiction. We infer that there exists a $k \in \mathbf{N}$ such that the angle at $y_{k}$ is close to $\pi$. In particular, if $x_{1}^{k}$ and $x_{2}^{k}$ are points in $C_{j}$ that realize the $\rho$-distance from $S_{j}$ at $y_{k}$, then $\rho$-distance between $x_{1}^{k}$ and $x_{2}^{k}$ is less than one. 
Next, suppose that $x_{i}^{k}$ and $x_{2}^{k}$ belong to the same connected component of $S_{i}$, for some $i \neq j$. Then, by Theorem 7.1 we know that $\partial S_{i} \cap \overline{\mathcal{B}_{1}\left(y_{k}\right)}$ has to contain the arc of the unit $\rho$-ball between $x_{1}^{k}$ and $x_{2}^{k}$. If not, there would be points in the curve connecting $x_{1}^{k}$ and $x_{2}^{k}$ which do not realize the distance from $C_{i}$. Any point inside this arc is a regular point at $\rho$-distance 1 from $y_{k}$. Consider any of them, for instance the middle point of the arc, denoted by $x_{k}$. We want to compare the mass of the Laplacian of $u_{i}$ at $x_{k}$ with the mass of the Laplacian at $u_{j}$ at $y_{k}$, across the free boundaries. Let us first assume $H$ defined as in 2.5 . For $\sigma<\frac{1}{8} d_{\rho}\left(x_{1}^{k}, x_{2}^{k}\right)$ let us define

$$
D_{\sigma}\left(x_{k}\right):=\left\{x \in \mathcal{B}_{\sigma}\left(x_{k}\right) \mid d\left(x, \partial \mathcal{C}_{i}\right) \leq \sigma^{2}\right\}
$$

where $\mathcal{C}_{i}$ is the asymptotic cone to $S_{i}^{1}$ at $x_{k}$. Note that, since $x_{k}$ is a regular point, $\partial \mathcal{C}_{i}$ is the tangent line to $\partial S_{i}^{1}$ at $x_{k}$, and so $\mathcal{C}_{i}$ has opening $\pi$. Let $\left(D_{\sigma}\left(x_{k}\right)\right)_{1}$ be the set of points at $\rho$-distance less than 1 from $D_{\sigma}\left(x_{k}\right)$, then we have that

$$
\int_{D_{\sigma}\left(x_{k}\right)} \Delta u_{i} \leq \sum_{j \neq i} \int_{\left(D_{\sigma}\left(x_{k}\right)\right)_{1}} \Delta u_{j}
$$

as in 7.2 with $\left(D_{\sigma}\left(x_{k}\right)\right)_{1}$ in place of $\mathcal{B}_{1+S}\left(x_{0}\right)$. By the Hopf Lemma, we obtain

$$
\int_{D_{\sigma}\left(x_{k}\right)} \Delta u_{i}=\int_{\partial S_{i} \cap D_{\sigma}\left(x_{k}\right)} \frac{\partial u_{i}}{\partial \nu_{i}} d \mathcal{H} \geq c \mathcal{H}\left(\partial S_{i} \cap D_{\sigma}\left(x_{k}\right)\right)=\tilde{C} \sigma
$$

where $\nu_{i}$ is the inner normal vector.

Now we estimate $\int_{\left(D_{\sigma}\left(x_{k}\right)\right)_{1}} \Delta u_{j}$. From Corollary 6.5 we know that $S_{j}$ is a set of finite perimeter. Therefore by Lemma 8.5 and Lemma 8.8 we obtain the following estimate

$$
\int_{\left(D_{\sigma}\left(x_{k}\right)\right)_{1}} \Delta u_{j}=\int_{\partial^{*} S_{j}^{1} \cap\left(D_{\sigma}\left(x_{k}\right)\right)_{1}} \frac{\partial u_{j}}{\partial \nu_{S_{j}^{1}}} d \mathcal{H} \leq C \sigma^{\beta_{\delta}} \mathcal{H}\left(\partial^{*} S_{j}^{1} \cap\left(D_{\sigma}\left(x_{k}\right)\right)_{1}\right)
$$

where $\nu_{S_{j}}$ is the measure-theoretic inward unit normal to $S_{j}^{1}$ and $\beta_{\delta}>0$. Since, for some constant $c$

$$
\partial S_{j}^{1} \cap\left(D_{\sigma}\left(x_{k}\right)\right)_{1} \subset \partial S_{j}^{1} \cap \mathcal{B}_{c \sigma}\left(y_{k}\right)
$$


by (2.2), there exists $\tilde{c}_{2}$, that for simplicity we will still name $c$, such that $\partial S_{j}^{1} \cap\left(D_{\sigma}\left(x_{k}\right)\right)_{1} \subset$ $\partial S_{j}^{1} \cap B_{c \sigma}\left(y_{k}\right)$. Then

$$
\mathcal{H}\left(\partial^{*} S_{j}^{1} \cap\left(D_{\sigma}\left(x_{k}\right)\right)_{1}\right) \leq \operatorname{Per}\left(\partial S_{j}^{1} \cap B_{c \sigma}\left(y_{k}\right)\right)
$$

To estimate $\operatorname{Per}\left(\partial S_{j}^{1} \cap B_{c \sigma}\left(y_{k}\right)\right)$, consider $(6.2)$ in the distributional sense. Then, take a smooth function $0 \leq \phi \leq 1$ with compact support contained in $B_{c \sigma}\left(y_{k}\right) \cap\left\{x: 0<d\left(x, S_{i}\right)<1\right\}$ and such that $\phi \equiv 1$ on the set $B_{c \sigma}\left(y_{k}\right) \cap\left\{x: 1-\delta<d\left(x, S_{i}\right)<1-\epsilon\right\}$, for $0<\varepsilon<\delta$ and $\delta$ as introduced in the definition of $\eta$ in the proof of Lemma 6.4. Then, for $d_{S_{i}}(\cdot)=d_{\rho}\left(\cdot, S_{i}\right)$ we have that

$$
\begin{aligned}
0=\int_{B_{c \sigma}\left(y_{k}\right) \cap\left\{x: 0<d_{S_{i}}<1\right\}} \operatorname{div}\left(\eta\left(d_{S_{i}}\right) D d_{S_{i}}\right) \phi & =\int_{B_{c \sigma}\left(y_{k}\right) \cap\left\{x: 0<d_{S_{i}}<1\right\}} \eta^{\prime}\left(d_{S_{i}}\right)\left|D d_{S_{i}}\right|^{2} \phi d x \\
& +\int_{B_{c \sigma}\left(y_{k}\right) \cap\left\{x: 0<d_{S_{i}}<1\right\}} \eta\left(d_{S_{i}}\right) \Delta d_{S_{i}} \phi \\
& \leq \int_{B_{c \sigma}\left(y_{k}\right) \cap\left\{x: 0<d_{S_{i}}<1\right\}} \eta^{\prime}\left(d_{S_{i}}\right)\left|D d_{S_{i}}\right|^{2} \phi d x+C \sigma .
\end{aligned}
$$

Proceeding as in Lemma 6.4 we obtain that

$$
\operatorname{Per}\left(\partial S_{j}^{1} \cap B_{c \sigma}\left(y_{k}\right)\right) \leq C \sigma
$$

Putting together 8.8, 8.9, 8.10, 8.11) and 8.12 we obtain

$$
C \sigma^{1+\beta_{\delta}} \geq \tilde{C} \sigma
$$

and we get a contradiction for $\sigma$ small enough. In the case 2.6 the proof follows the same steps using (7.8).

Therefore we must have that $x_{1}^{k}$ and $x_{2}^{k}$ belong to different components of $C_{j}$ for any $k \geq \bar{k}$. In particular, since the distance between them is less than one, they must belong to two different components of the same population. Suppose that $x_{1}^{\bar{k}} \in S_{i}^{1}$ and $x_{2}^{\bar{k}} \in S_{i}^{2}$, for $i \neq j$. Consider the consecutive two points $x_{1}^{\bar{k}+1}$ and $x_{2}^{\bar{k}+1}$ which realize the distance at $y_{\bar{k}+1}$, and again belong to two different components of $C_{j}$. Since $S_{j}^{1}$ (to which $y_{\bar{k}}$ belongs) and $S_{i}^{2}$ reach the boundary of $\Omega$, the point $x_{2}^{\bar{k}+1}$ must belong to a connected component different from $S_{i}^{1}$. Iterating the 
procedure, we construct a sequence of distinct points belonging to connected components, each different from the others. This is in contradiction with Lemma 8.4. We conclude that singular points are isolated.

Theorem 8.10. Assume (2.8) with $n=2$ and $p=1$ in 2.5 . Let $\left(u_{1}^{\varepsilon}, \ldots, u_{K}^{\varepsilon}\right)$ be a viscosity solution of the problem (2.4) and $\left(u_{1}, \ldots, u_{K}\right)$ the limit as $\varepsilon \rightarrow 0$ of a convergent subsequence. For $i \neq j$, let $x_{0} \in \partial S_{i} \cap \Omega$ and $y_{0} \in \partial S_{j} \cap \Omega$ be points such that $S_{i}$ has an angle $\theta_{i} \in[0, \pi]$ at $x_{0}, S_{j}$ has an angle $\theta_{j} \in[0, \pi]$ at $y_{0}$ and $\rho\left(x_{0}-y_{0}\right)=1$. Then we have

$$
\theta_{i}=\theta_{j}
$$

If $x_{0} \in \partial S_{i} \cap \partial \Omega$ and $y_{0} \in \partial S_{j} \cap \Omega$, then

$$
\theta_{i} \leq \theta_{j}
$$

Proof. By Lemma 8.4, the connected components of the sets $S_{i}$ 's are finite. Assume $x_{0} \in \bar{\Omega}$ and $y_{0} \in \Omega$. Without loss of generality we can assume that $x_{0}=0$. It suffices to show the theorem for $y_{0}$ belonging to a region that is side by side with $S_{i}$, in the sense that 0 is the limit as $h \rightarrow 0$ of interior regular points $x_{h} \in \partial S_{i} \cap \Omega$ with the property that $x_{h}$ realizes the distance from $S_{j}$ at $y_{h} \in \partial S_{j} \cap \Omega$ interior points, with $y_{h} \rightarrow y_{0}$ as $h \rightarrow 0$. Let $\mathcal{C}_{i}$ be the asymptotic cone at 0 . Let us first suppose for simplicity that $\partial S_{i}$ and $\partial S_{j}$ are locally a cone around 0 and $y_{0}$ respectively. In particular, $\theta_{i}, \theta_{j}>0$. We will explain later on how to handle the general case.

Proof of Theorem 8.10 when $\partial S_{i}$ and $\partial S_{j}$ are locally cones. We assume that there exists $r_{0}>0$ such that $\partial S_{i} \cap B_{2 r_{0}}=\mathcal{C}_{i} \cap B_{2 r_{0}}$, where $B_{2 r_{0}}$ is the Euclidian ball centered at 0 of radius $2 r_{0}$. When $x_{0} \in \partial \Omega$ we are just interested in the side of the cone $\mathcal{C}_{i}$ contained in $\Omega$.

If $(\varrho, \theta)$ is a system of polar coordinates in the plane centered at zero, we may assume that $\mathcal{C}_{i}$ is the cone given by

$$
\mathcal{C}_{i}=\left\{(\varrho, \theta) \mid \varrho \in[0,+\infty), 0 \leq \theta \leq \theta_{i}\right\}
$$


Let us first consider the case 2.6. Let us assume that $x_{h}=\left(2 r_{h}, 0\right)$, with $r_{h}>0$. We know that $r_{h} \rightarrow 0$ as $h \rightarrow 0$, then we can fix $h$ so small that $r_{h}<r_{0} / 3$. By Lemma 8.6 applied to $u_{1}=u_{i}$, we have

$$
u_{i}(x) \geq c r_{h}^{\alpha} d\left(x, \partial S_{i}\right) \quad \text { for any } x \in\left[r_{h}, 3 r_{h}\right] \times\left[0, R_{h}\right]
$$

where

$$
1+\alpha=\frac{\pi}{\theta_{i}} \geq 1
$$

Now, we repeat an argument similar to the one in the proof of Theorem 7.1. We look at inf $u_{i}$ in small circles of radius $r$ that go across the free boundary of $u_{i}$ and we look at sup $u_{j}$ in circles of radius $r+1$ across the free boundary of $u_{j}$, then we compare the mass of the correspondent Laplacians. Precisely, there exists a small $\sigma>0$ and $e \in S_{i}$ such that $\mathcal{B}_{\sigma}(e) \subset\left[r_{h}, 3 r_{h}\right] \times\left[0, R_{h}\right]$ and $x_{h} \in \partial \mathcal{B}_{\sigma}(e)$. In particular, in $\mathcal{B}_{\sigma}(e)$ the function $u_{i}$ satisfies 8.15. For $v<\sigma$ and $r \in[\sigma-v, \sigma+v]$, we define

$$
\underline{u}_{i}:=\inf _{\partial \mathcal{B}_{r}(e)} u_{i} \quad \text { and } \quad \bar{u}_{j}:=\sup _{\partial \mathcal{B}_{1+r}(e)} u_{j} .
$$

In what follows we denote by $C$ and $c$ several constants independent of $h$. For $r \in[\sigma-v, \sigma]$, by 8.15 we have

$$
\underline{u}_{i} \geq \inf _{\partial \mathcal{B}_{r}(e)} c r_{h}^{\alpha} d\left(x, \partial S_{i}\right) \geq \inf _{\partial \mathcal{B}_{r}(e)} C r_{h}^{\alpha} d_{\rho}\left(x, \partial S_{i}\right) \geq C r_{h}^{\alpha}(\sigma-r)
$$

For $r \in[\sigma, \sigma+v]$, the ball $\mathcal{B}_{r}(e)$ goes across $\partial S_{i}$, therefore we have $\underline{u}_{i}=0$. Hence

$$
\begin{aligned}
& \underline{u}_{i}(r) \geq C r_{h}^{\alpha}(\sigma-r) \quad \text { for } r \in[\sigma-v, \sigma] \\
& \underline{u}_{i}(r)=0 \quad \text { for } r \in[\sigma, \sigma+v] .
\end{aligned}
$$

Next, let us study the behavior of $\bar{u}_{j}$. First of all, let us show that

$$
d_{\rho}\left(e, \partial S_{j}\right)=\rho\left(e-y_{h}\right)=1+\sigma .
$$

Since $d_{\rho}\left(e, \partial S_{i}\right)=\sigma$ and $d_{\rho}\left(S_{i}, S_{j}\right) \geq 1$, it is easy to see that $d_{\rho}\left(x, \partial S_{j}\right) \geq 1+\sigma$. The function $\rho$ is also called a Minkowski norm and from known results about Minkowski norms, if we denote by $T$ the Legendre transform $T: \mathbb{R}^{n} \rightarrow \mathbb{R}^{n}$ defined by $T(y)=\rho(y) D \rho(y)$, then $T$ is a bijection with 
inverse $T^{-1}(\xi)=\rho^{*}(\xi) D \rho^{*}(\xi)$, where $\rho^{*}$ is the dual norm defined by $\rho^{*}(\xi):=\sup \left\{y \cdot \xi \mid y \in \mathcal{B}_{1}\right\}$. Now, the ball $\mathcal{B}_{1}\left(y_{h}\right)$ is tangent to $\partial S_{i}$ at $x_{h}$ and therefore is also tangent to $\mathcal{B}_{\sigma}(e)$ at $x_{h}$. This implies that $D \rho\left(e-x_{h}\right)=-D \rho\left(x_{h}-e\right)=D \rho\left(x_{h}-y_{h}\right)$. Consequently we have

$$
\begin{aligned}
e-x_{h} & =T^{-1}\left(T\left(e-x_{h}\right)\right)=T^{-1}\left(\sigma D \rho\left(e-x_{h}\right)\right)=T^{-1}\left(\sigma D \rho\left(x_{h}-y_{h}\right)\right) \\
& =\sigma T^{-1}\left(T\left(x_{h}-y_{h}\right)=\sigma\left(x_{h}-y_{h}\right) .\right.
\end{aligned}
$$

We infer that

$$
e=x_{h}+\sigma\left(x_{h}-y_{h}\right)
$$

and

$$
\rho\left(e-y_{h}\right)=(1+\sigma) \rho\left(x_{h}-y_{h}\right)=1+\sigma
$$

which proves 8.19). As a consequence $\partial \mathcal{B}_{1+r}(e) \cap S_{j}=\emptyset$ for $r \in[\sigma-v, \sigma)$, while if $r \in(\sigma, \sigma+v]$ then $\partial \mathcal{B}_{1+r}(e) \cap S_{j} \neq \emptyset$ and $\partial \mathcal{B}_{1+r}(e)$ enters inside $S_{j}$ at $\rho$-distance at most $r-\sigma$ from the boundary of $S_{j}$. In particular we have

$$
\bar{u}_{j}=0 \quad \text { for } r \in[\sigma-v, \sigma]
$$

Next, if $\theta_{j}$ is the angle of $S_{j}$ at $y_{0}$, let $\beta$ be defined by

$$
1+\beta=\frac{\pi}{\theta_{j}} \geq 1
$$

Remark that $y_{h}$ is at $\rho$-distance $2 r_{h}$ from $y_{0}$. Again by Lemma 8.6 applied to $u_{2}=u_{j}$, (after a rotation and a translation), we have the following estimate

$$
u_{j}(x) \leq C r_{h}^{\beta} d\left(x, \partial S_{j}\right) \leq C r_{h}^{\beta} d_{\rho}\left(x, \partial S_{j}\right),
$$

in a neighborhood of $y_{h}$. As a consequence, recalling in addition that the ball $\mathcal{B}_{1+r}(e)$ enters in $S_{j}$ at $\rho$-distance $r-\sigma$ from the boundary, for $r \in[\sigma, \sigma+v]$ we get

$$
\bar{u}_{j}=\sup _{\partial \mathcal{B}_{1+r}(e)} u_{j} \leq C r_{h}^{\beta}(r-\sigma) .
$$

The last estimate and (8.21) imply

$$
\bar{u}_{j}(r) \leq C r_{h}^{\beta}(r-\sigma)^{+}, \quad \text { for } r \in[\sigma-v, \sigma+v]
$$


Now, we want to compare the mass of the Laplacians of $\underline{u}_{i}$ and $\bar{u}_{j}$. Define as in (8.17)

$$
\underline{u}_{i}^{\varepsilon}:=\inf _{\partial \mathcal{B}_{r}(e)} u_{i}^{\varepsilon}, \quad \bar{u}_{k}^{\varepsilon}:=\sup _{\partial \mathcal{B}_{1+r}(e)} u_{k}^{\varepsilon}, k \neq i .
$$

For $\sigma$ and $v$ small enough, the ball $\mathcal{B}_{r}(e)$ is contained in $\Omega$ for any $r \leq \sigma+v$, and thus

$$
\Delta u_{i}^{\varepsilon}=\frac{1}{\varepsilon^{2}} u_{i}^{\varepsilon} \sum_{k \neq i} H\left(u_{k}^{\varepsilon}\right) \quad \text { in } \mathcal{B}_{r+\sigma}(e) .
$$

On the other hand, since $x_{h}$ is an interior regular point that realizes its distance from $S_{j}$ at an interior point, $y_{h}$, its distance from the support of the boundary data $f_{k}$ is greater than 1 , for any $k \neq i$. We infer that, for $\sigma$ and $v$ small enough and $r \leq \sigma+v$,

$$
\Delta u_{k}^{\varepsilon} \geq \frac{1}{\varepsilon^{2}} u_{k}^{\varepsilon} \sum_{l \neq k} H\left(u_{l}^{\varepsilon}\right) \quad \text { in } \mathcal{B}_{1+r}(e)
$$

Hence, arguing as in the proof of Theorem 7.1, we see that

$$
\Delta_{r} \underline{u}_{i}^{\varepsilon} \leq \sum_{k \neq i} \Delta_{r} \bar{u}_{k}^{\varepsilon} \quad \text { in }(\sigma-v, \sigma+v)
$$

where $\Delta_{r} u=\frac{1}{r} \frac{\partial}{\partial r}\left(r \frac{\partial u}{\partial r}\right)$. Since $x_{h}$ is a regular point of $\partial S_{i}$ that realizes the distance from $S_{j}$ at $y_{h} \in \partial C_{i}$, the ball $\mathcal{B}_{1+\sigma+v}(e)$ does not intersect the support of the functions $u_{k}$ for $k \neq j$ and small $v$ and $\sigma$. Therefore, multiplying inequality 8.24 by a positive test function $\phi \in C_{c}^{\infty}(\sigma-v, \sigma+v)$, integrating by parts in $(\sigma-v, \sigma+v)$ and passing to the limit as $\varepsilon \rightarrow 0$ along a converging subsequence, the only surviving function on the right-hand side is $\bar{u}_{j}$ and we get

$$
\int_{\sigma-v}^{\sigma+v} \underline{u}_{i} \frac{\partial}{\partial r}\left(r \frac{\partial}{\partial r}\left(\frac{1}{r} \phi\right)\right) d r \leq \int_{\sigma-v}^{\sigma+v} \bar{u}_{j} \frac{\partial}{\partial r}\left(r \frac{\partial}{\partial r}\left(\frac{1}{r} \phi\right)\right) d r .
$$

Let us choose a function $\phi$ which is increasing and $(\sigma-v, \sigma)$ and decreasing in $(\sigma, \sigma+v)$ and hence with maximum at $r=\sigma$, and let us estimates the left and the right hand-side of the last 
inequality. Estimates 8.18 imply that $\frac{\partial \underline{u}_{i}}{\partial r}\left(\sigma^{-}\right) \leq-C r_{h}^{\alpha}$. Therefore, for small $v$ we have

$$
\begin{aligned}
\int_{\sigma-v}^{\sigma+v} \underline{u}_{i} \frac{\partial}{\partial r}\left(r \frac{\partial}{\partial r}\left(\frac{1}{r} \phi\right)\right) d r & =-\int_{\sigma-v}^{\sigma} \frac{\partial \underline{u}_{i}}{\partial r} r \frac{\partial}{\partial r}\left(\frac{1}{r} \phi\right) d r \\
& =-\int_{\sigma-v}^{\sigma}\left(\frac{\partial \underline{u}_{i}}{\partial r}\left(\sigma^{-}\right)+o_{\sigma-r}(1)\right) r \frac{\partial}{\partial r}\left(\frac{1}{r} \phi\right) d r \\
& \geq-\int_{\sigma-v}^{\sigma} \frac{\partial \underline{u}_{i}}{\partial r}\left(\sigma^{-}\right)\left(\frac{\partial \phi}{\partial r}-\frac{1}{r} \phi\right) d r \\
& -o_{v}(1) \int_{\sigma-v}^{\sigma}\left(\frac{\partial \phi}{\partial r}+\frac{1}{r} \phi\right) d r \\
& \geq-\frac{\partial \underline{u}_{i}}{\partial r}\left(\sigma^{-}\right)\left[\phi(\sigma)-\phi(\sigma) \log \left(\frac{\sigma}{\sigma-v}\right)\right] \\
& -o_{v}(1)\left[\phi(\sigma)+\phi(\sigma) \log \left(\frac{\sigma}{\sigma-v}\right)\right] \\
& \geq\left(C r_{h}^{\alpha}-o_{v}(1)\right) \phi(\sigma) .
\end{aligned}
$$

Similarly, using (8.23) and integrating by parts, we get

$$
\int_{\sigma-v}^{\sigma+v} \bar{u}_{j} \frac{\partial}{\partial r}\left(r \frac{\partial}{\partial r}\left(\frac{1}{r} \phi\right)\right) d r \leq\left(C r_{h}^{\beta}+o_{v}(1)\right) \phi(\sigma) .
$$

From the previous estimates and 8.25 , letting $v$ go to 0 , we obtain

$$
r_{h}^{\alpha} \leq C r_{h}^{\beta}
$$

and therefore, for $h$ small enough

$$
\beta \leq \alpha
$$

Recalling the definitions 8.16 and 8.22 of $\alpha$ and $\beta$ respectively, we infer that

$$
\theta_{i} \leq \theta_{j}
$$

This proves 8.14. If $x_{0}=0$ is an interior point of $\Omega$, exchanging the roles of $u_{i}$ and $u_{j}$, we get the opposite inequality

$$
\theta_{j} \leq \theta_{i}
$$

and this proves 8.13 for $H$ defined as in 2.6.

Next, let us turn to the case 2.5. Again we compare the mass of Laplacians of $u_{i}$ and $u_{j}$ across the free boundaries. For $\sigma<r_{h}$ let us define

$$
D_{\sigma}\left(x_{h}\right):=\left\{x \in \mathcal{B}_{\sigma}\left(x_{h}\right) \mid d\left(x, \partial S_{i}\right) \leq \sigma^{2}\right\} .
$$


Then, if we denote by $\left(D_{\sigma}\left(x_{h}\right)\right)_{1}$ the sets of points at $\rho$-distance less than 1 , we have that

$$
\int_{D_{\sigma}\left(x_{h}\right)} \Delta u_{i} \leq \sum_{k \neq i} \int_{\left(D_{\sigma}\left(x_{h}\right)\right)_{1}} \Delta u_{k}
$$

as in 7.2 with $\left(D_{\sigma}\left(x_{h}\right)\right)_{1}$ in place of $\mathcal{B}_{1+S}\left(x_{0}\right)$. By Lemma 8.6 the normal derivative of $u_{i}$ with respect to the inner normal $\nu_{i}$, at any point on the boundary $\partial \mathcal{C}_{i}$ with distance to the vertex between $r_{h}$ and $3 r_{h}$ is greater than $c r_{h}^{\alpha}$, then

$$
\int_{D_{\sigma}\left(x_{h}\right)} \Delta u_{i}=\int_{\partial \mathcal{C}_{i} \cap D_{\sigma}\left(x_{h}\right)} \frac{\partial u_{i}}{\partial \nu_{i}} d \mathcal{H} \geq c \int_{2 r_{h}-c \sigma}^{2 r_{h}+C \sigma} r_{h}^{\alpha} d r=C r_{h}^{\alpha} \sigma .
$$

Remark that

$$
\left(D_{\sigma}\left(x_{h}\right)\right)_{1} \cap \partial S_{j} \subset \mathcal{B}_{c \sigma}\left(y_{h}\right) \cap \partial S_{j}
$$

therefore, for $\sigma$ small enough, again from Lemma 8.6 we have

$$
\int_{\left(D_{\sigma}\left(x_{h}\right)\right)_{1}} \Delta u_{j} \leq C r_{h}^{\beta} \sigma
$$

Then for $r_{h}$ small enough we obtain that

$$
\beta \leq \alpha
$$

and therefore

$$
\theta_{i} \leq \theta_{j}
$$

If $x_{0}=0$ is an interior point of $\Omega$, exchanging the roles of $u_{i}$ and $u_{j}$ we get the opposite inequality

$$
\theta_{j} \leq \theta_{i}
$$

This concludes the proof of the theorem in the particular case in which $\partial S_{i}$ and $\partial S_{j}$ are locally a cone around 0 and $y_{0}$ respectively.

We are now going to explain how to adapt the proof in the general case.

Proof of Theorem 8.10 in the general case. If $\theta_{i}=0$, then $\theta_{i} \leq \theta_{j}$. Assume $\theta_{i} \in(0, \pi]$ and $\theta_{j} \in[0, \pi]$, then for any $0<\delta<\theta_{i}$, there exist $r_{\delta}>0$, a cone $\mathcal{C}_{\delta}^{i}$ centered at 0 and with opening 
$\theta_{i}-\delta$, and a cone $\mathcal{C}_{\delta}^{j}$ centered at $y_{0}$ and with opening $\theta_{j}+\delta$ such that

$$
\mathcal{C}_{\delta}^{i} \cap B_{r_{\delta}}(0) \subset S_{i} \cap B_{r_{\delta}}(0) \quad \text { and } \quad S_{j} \cap B_{r_{\delta}}\left(y_{0}\right) \subset \mathcal{C}_{\delta}^{j} \cap B_{r_{\delta}}\left(y_{0}\right)
$$

Let $\left(x_{h}\right)_{h}$ be the sequence of regular points on $\partial S_{i} \cap \Omega$ given by Lemma 8.7 (consider $\Gamma_{1}$ the closest side to $\left.S_{j}\right)$ and let $r_{h}=d\left(0, x_{h}\right)$. Denote by $y_{h}$ the point on $\partial S_{j} \cap \Omega$ at $\rho$-distance 1 from $x_{h}$. Then, $d_{\rho}\left(y_{h}, y_{0}\right) \leq c r_{h}$. Now, the proof of the theorem proceeds like in the previous case and we can compare the mass of the laplacians across the free boundaries of $u_{i}$ and $u_{j}$.

Let us first consider the case (2.5). For $\sigma>0$ take $D_{\sigma}\left(x_{h}\right)$ and $\left(D_{\sigma}\left(x_{h}\right)\right)_{1}$ as defined as in 8.26). For $\sigma$ small enough, by Lemma $8.9 \partial S_{i} \cap D_{\sigma}\left(x_{h}\right)$ does not contain singular points and by Lemma 8.3 it is a $C^{1}$ curve of the plane.

By Lemma 8.7

$$
\int_{D_{\sigma}\left(x_{h}\right)} \Delta u_{i}=\int_{\partial S_{i} \cap D_{\sigma}\left(x_{h}\right)} \frac{\partial u_{i}}{\partial \nu_{i}} d \mathcal{H} \geq C r_{h}^{\alpha_{\delta}} \sigma
$$

Remark that

$$
\left(D_{\sigma}\left(x_{h}\right)\right)_{1} \cap \partial S_{j} \subset \mathcal{B}_{c \sigma}\left(y_{h}\right) \cap \partial S_{j}
$$

therefore, for $\sigma$ small enough, from Lemma 8.8, as in the proof of Lemma 8.9, we have

$$
\int_{\left(D_{\sigma}\left(x_{h}\right)\right)_{1}} \Delta u_{j} \leq \tilde{C} r_{h}^{\beta_{\delta}} \sigma
$$

Then for $h$ small enough, we obtain that

$$
\beta_{\delta} \leq \alpha_{\delta}
$$

and therefore

$$
\theta_{i} \leq \theta_{j}
$$

If $x_{0}=0$ is an interior point of $\Omega$, exchanging the roles of $u_{i}$ and $u_{j}$ we get the opposite inequality

$$
\theta_{j} \leq \theta_{i}
$$

Next, let us turn to the case (2.6). Then, we define, for $r \in\left[R_{h}-v, R_{h}+v\right]$,

$$
\underline{u}_{i}:=\inf _{\partial \mathcal{B}_{r}\left(z_{h}\right)} u_{i} \quad \text { and } \quad \bar{u}_{j}:=\sup _{\partial \mathcal{B}_{1+r}\left(z_{h}\right)} u_{j}
$$


Arguing as before, and using the Lemma 8.7 we get

$$
\beta_{\delta} \leq \alpha_{\delta}
$$

and therefore, letting $\delta$ go to 0 , we finally obtain

$$
\theta_{i} \leq \theta_{j}
$$

Remark in particular that if $\theta_{i}>0$ then $\theta_{j}>0$. If $x_{0}=0$ is an interior point of $\Omega$, exchanging the roles of $u_{i}$ and $u_{j}$ we get the opposite inequality $\theta_{j} \leq \theta_{i}$.

An immediate corollary of Theorem 8.10 is the $C^{1}$-regularity of the free boundaries when $K=2$ and under the following additional assumptions on $\Omega, f_{1}$ and $f_{2}$ :

$$
\Omega:=\left\{\left(x_{1}, x_{2}\right) \in \mathbb{R}^{2} \mid g\left(x_{2}\right) \leq x_{1} \leq h\left(x_{2}\right), x_{2} \in[a, b]\right\}, \quad b-a \geq 4
$$

where

$$
\left\{\begin{array}{l}
g, h:[a, b] \rightarrow \mathbb{R} \text { are Lipschitz functions with } \\
-m_{2} \leq g \leq-m_{1} \leq M_{2} \leq h \leq M_{1}, \quad M_{2} \geq-m_{1}+4
\end{array}\right.
$$

the boundary data are such that

$$
\left\{\begin{array}{l}
f_{1} \equiv 1, f_{2} \equiv 0 \quad \text { on }\left\{x_{1} \leq g\left(x_{2}\right)\right\} \\
f_{1} \equiv 0, f_{2} \equiv 1 \quad \text { on }\left\{x_{1} \geq h\left(x_{2}\right)\right\} \\
f_{1} \text { is monotone decreasing in } x_{1} \text { on }\left\{x_{2} \leq a\right\} \cup\left\{x_{2} \geq b\right\} \\
f_{2} \text { is monotone increasing in } x_{1} \text { on }\left\{x_{2} \leq a\right\} \cup\left\{x_{2} \geq b\right\}
\end{array}\right.
$$

These assumptions imply that $-u_{1}$ and $u_{2}$ are monotone increasing in the $x_{1}$ direction. Then we have the following

Corollary 8.11. Assume (2.8) with $p=1$ in (2.5). Assume in addition $K=n=2$, 8.28), (8.29) and (8.30). Then the sets $\partial S_{i}, i=1,2$, are of class $C^{1}$.

Proof. We know that the sets $\partial S_{i}$ are curves of the plane at $\rho$-distance 1 , one from each other. Suppose by contradiction that $\partial S_{1}$ has an angle $\theta<\pi$ at $y_{0}$. In particular, there exist two $\rho$-balls of radius 1 , centered at two points $z, w \in \partial S_{2}$ that are tangent to $\partial S_{1}$ at $y_{0}$. Then, by the monotonicity property of the $u_{i}$ 's and Theorem 7.1, the arc of the $\rho$-ball of radius 1 centered 
at $y_{0}$ between the points $z$ and $w$ must be all in $\partial S_{2}$. This means that any point inside this arc, which is a regular point of $\partial S_{2}$, is at $\rho$-distance 1 from the singular point $y_{0} \in \partial S_{1}$. This contradicts Theorem 8.10 . We have shown that any point of the free boundaries is regular. Then by Lemma 8.3 the free boundaries are of class $C^{1}$. This concludes the proof.

Another corollary of Theorem 8.10 is that the number of singular points is finite.

Corollary 8.12. Assume $(2.8)$ with $n=K=2$ and $p=1$ in (2.5). Assume in addition that the supports on $\partial \Omega$ of the boundary data $f_{1}$ and $f_{2}$ have a finite number of connected components. Then singular points form a finite set.

Proof. From Lemma 8.4, $S_{1}$ and $S_{2}$ have a finite number of connected components. Moreover, we recall that any connected component has to reach the boundary.

Let $x_{0}$ be a singular point belonging to the boundary of the support of one of the limit functions $u_{i}$. W.l.o.g. let us assume $x_{0} \in \partial S_{1}$. Let $y_{1}, y_{2} \in \partial S_{2}$ two different points where $x_{0}$ realizes the distance from $S_{2},\left(y_{1}, y_{2} \in \partial \mathcal{B}_{1}\left(x_{0}\right) \cap \partial S_{2}\right.$, see Figure 3). We can choose $y_{1}$ such that $\mathcal{B}_{1}\left(x_{0}\right)$ is the limit as $k \rightarrow+\infty$ of balls $\mathcal{B}_{1}\left(x_{k}\right)$ with $x_{k} \in \partial S_{1}$, tangent to points $y_{k} \in \partial S_{2}$ with $y_{k} \rightarrow y_{1}$ and $x_{k} \rightarrow x_{0}$ as $k \rightarrow+\infty$. Theorem 8.10 implies that $S_{2}$ has an angle at $y_{1}$ and $y_{2}$ and the intersection of the arc on $\partial \mathcal{B}_{1}\left(x_{0}\right)$ between $y_{1}$ and $y_{2}$ with $\partial C_{1}$ must have empty interior. This means that near $y_{1}$ there are points on $\partial S_{2}$ outside $\overline{\mathcal{B}_{1}\left(x_{0}\right)}$. These points are at distance greater than 1 from $x_{0}$ and from any other point of $\partial S_{1}$ close to $x_{0}$ and must realize the distance from $S_{1}$ outside $\mathcal{B}_{1}\left(y_{1}\right)$, see Figure 3. Therefore if we take a sequence $z_{k}$ of such points converging to $y_{1}$ and we consider the corresponding tangent balls centered at points that are in $\partial S_{1}$ where the $z_{k}$ 's realize the distance, we obtain a second tangent ball $\mathcal{B}_{1}\left(x_{1}\right)$ for $y_{1}$ with $x_{1} \neq x_{0}$.

Now, let us denote by $S_{1}^{1}$ the connected component of $S_{1}$ whose boundary contains $x_{0}$. Remember that since $S_{1}$ and $S_{2}$ are at $\rho$-distance 1 , we have $u_{1} \equiv 0$ in $\overline{\mathcal{B}_{1}\left(y_{1}\right)} \cup \overline{\mathcal{B}_{1}\left(y_{2}\right)}$. 


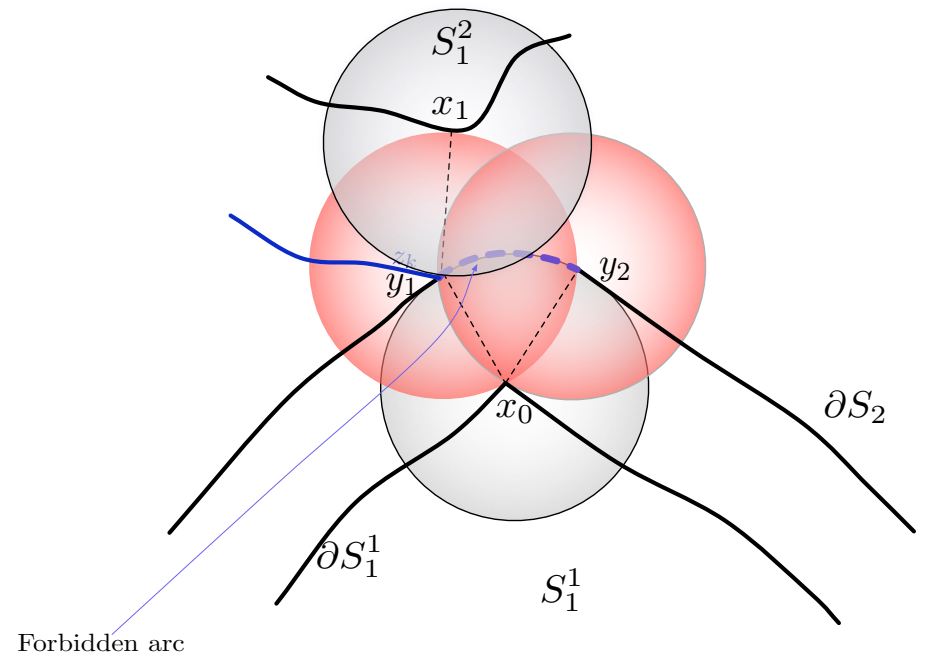

FiguRE 3. Forbidden arc

Moreover, since the connected components of $S_{2}$ whose boundaries contain $y_{1}$ and $y_{2}$ must reach the boundary of $\Omega$, they separate the components of $S_{1}$ whose boundaries contain $x_{0}$ and $x_{1}$. Therefore $x_{1}$ must belong to the boundary of different components of $S_{1}$. The same argument that we have used for $x_{1}$ and $x_{0}$ proves also that $y_{1}$ and $y_{2}$ must belong to the boundary of different components of $C_{1}$.

We conclude that a singular point $x_{0}$ of $S_{1}$ involves at least four different connected components and there correspond to it another singular point, $x_{1}$, belonging to a different component of $S_{1}$ (see Figure 4). Assume w.l.o.g. that $x_{1} \in \partial S_{1}^{2}$. Since all the connected components must reach the boundary of $\Omega, x_{1}$ is the only singular point of $S_{1}^{2}$ corresponding to a singular point of $S_{1}^{1}$. Since the connected component of $S_{1}$ are finite, we infer that there is a finite number of singular points on $\partial S_{1}^{1}$. This argument applied to any connected component of $S_{1}$ shows that singular points of $S_{1}$ are finite. This concludes the proof of the theorem.

8.2. Lipschitz regularity of the free boundaries. In this section, we will show, under some additional assumptions on the domain $\Omega$ and the boundary data $f_{i}$, that we can construct a 


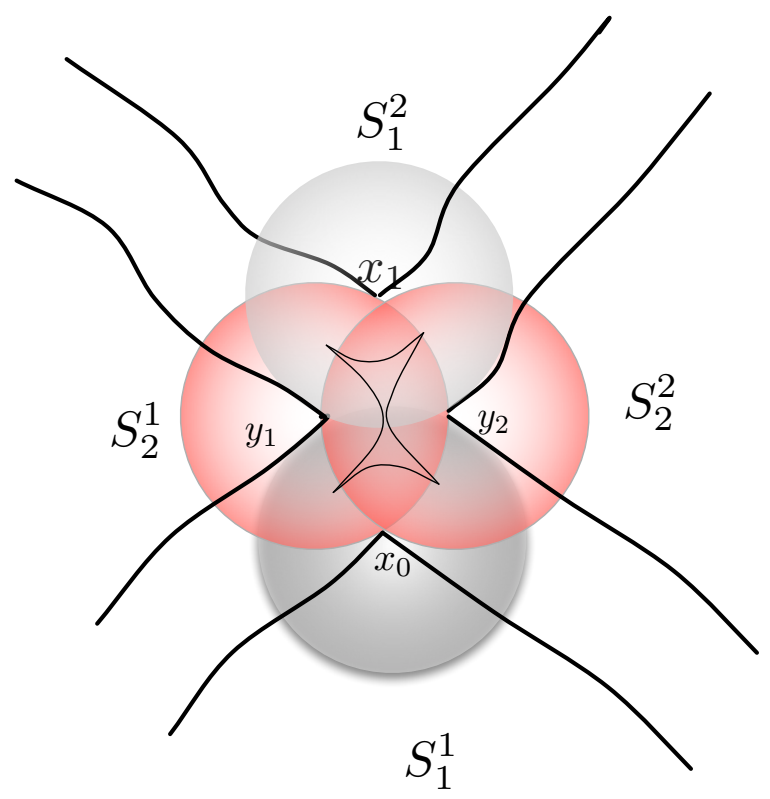

FiguRE 4. A singular point involving four components

solution of problem (2.4) such that the free boundaries $S_{i}$ of the limiting functions have the following properties: if $S_{i}$ has an angle $\theta$ at a singular point, then $\theta>0$. This result can be rephrased by saying that the free boundaries are Lipschitz curves of the plane. Let us make the assumptions precise. We assume that the domain $\Omega$ has the property that for any point of the boundary there are tangent $\rho$-balls of radius $1+\eta$, with $\eta>0$ contained in $\Omega$ and in its complementary. Precisely:

$$
\left\{\begin{array}{l}
\Omega \text { is a bounded domain of } \mathbb{R}^{2} ; \\
\quad \exists \eta>0 \text { such that } \forall x \in \partial \Omega, \exists \mathcal{B}_{1+\eta}(y), \mathcal{B}_{1+\eta}(z) \text { such that } \\
\quad x \in \partial \mathcal{B}_{1+\eta}(y) \cap \partial \mathcal{B}_{1+\eta}(z), \mathcal{B}_{1+\eta}(y) \subset \Omega, \text { and } \mathcal{B}_{1+\eta}(z) \subset \Omega^{c} .
\end{array}\right.
$$

On the boundary data $f_{i}, i=1, \ldots, K$, we assume,

$$
\left\{\begin{array}{l}
f_{i} \equiv 1 \text { in supp } f_{i} ; \\
\exists c>0 \text { s. t. } \forall x \in \partial \Omega \cap \operatorname{supp} f_{i},\left|\mathcal{B}_{r}(x) \cap \operatorname{supp} f_{i}\right| \geq c\left|\mathcal{B}_{r}(x)\right|, \\
d_{\rho}\left(\operatorname{supp} f_{i}, \operatorname{supp} f_{j}\right) \geq 1, i \neq j, \\
d_{\rho}\left(\operatorname{supp} f_{i} \cap \partial \Omega, \operatorname{supp} f_{i+1} \cap \partial \Omega\right)=1, \text { where } f_{K+1}:=f_{1} ; \\
\Gamma_{i}:=\operatorname{supp} f_{i} \cap \partial \Omega \text { is a connected }\left(C^{2}\right) \text { curve of } \partial \Omega .
\end{array}\right.
$$

We are going to build a solution of (2.4) such that the support of any limiting function $u_{i}$ contains a full neighborhood of $\Gamma_{i}$ in $\Omega$ with Lipschitz boundary. Then we prove that the free 


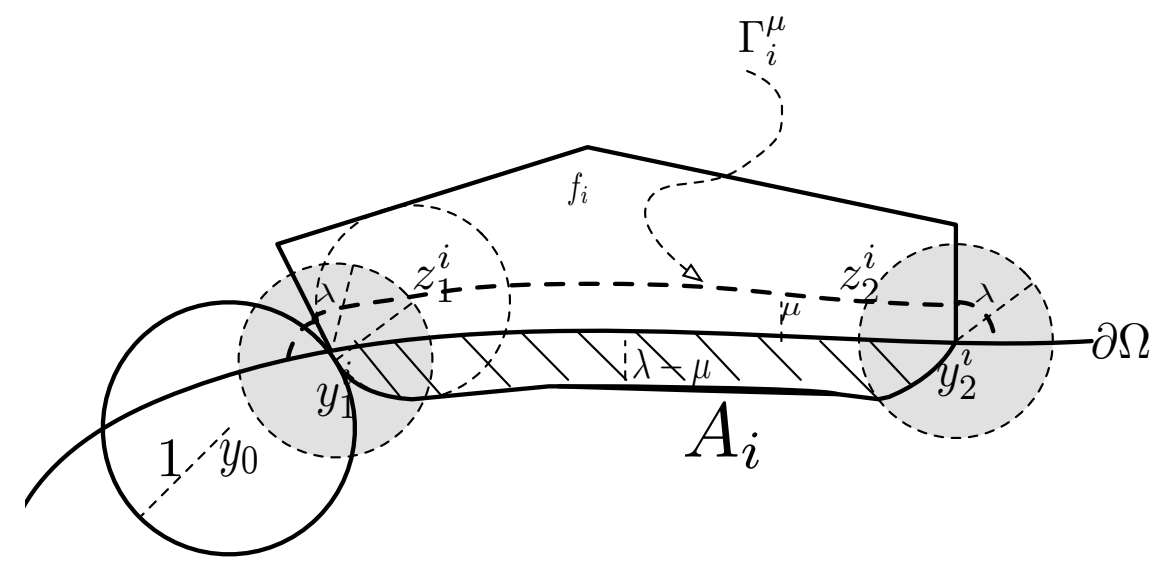

Figure 5. Construction of obstacle

boundaries are Lipschitz. In order to do it, we first prove the existence of a solution $\left(u_{1}^{\varepsilon}, \ldots, u_{K}^{\varepsilon}\right)$ of an obstacle problem associated to system 2.4. Then we show that the functions $u_{i}^{\varepsilon}$ 's never touch the obstacles, implying that $\left(u_{1}^{\varepsilon}, \ldots, u_{K}^{\varepsilon}\right)$ is actually solution of $(2.4)$. We consider obstacle functions $\psi_{i}$, for $i=1, \ldots, K$ defined as follows. Let $y_{1}^{i}, y_{2}^{i}$ be the endpoints of the curve $\Gamma_{i}$. For $0<\mu<\lambda<1$, we set:

$$
\begin{gathered}
\Gamma_{i}^{\mu}:=\left\{x \in \Omega^{c} \mid d\left(x, \Gamma_{i}\right)=\mu\right\}, \\
\Gamma_{i}^{\mu, \lambda}:=\left\{x \in \Gamma_{i}^{\mu} \mid d\left(x, y_{1}^{i}\right), d\left(x, y_{2}^{i}\right) \geq \lambda\right\} .
\end{gathered}
$$

For $\mu$ and $\lambda$ small enough, $\Gamma_{i}^{\mu, \lambda}$ is a $C^{1,1}$ curve of $\Omega^{c}$ with endpoints $z_{1}^{i}, z_{2}^{i}$ such that $d\left(z_{l}^{i}, y_{l}^{i}\right)=\lambda$, $l=1,2$. We finally set

$$
A_{i}:=\left\{x \in \Omega \mid d\left(x, \Gamma_{i}^{\mu, \lambda}\right)<\lambda\right\}=\Omega \cap\left(\cup_{x \in \Gamma_{i}^{\mu, \lambda}} B_{\lambda}(x)\right) .
$$

Remark that

$$
\partial A_{i}=\Gamma_{i} \cup\left(\partial A_{i} \cap \Omega\right),
$$


where $\partial A_{i} \cap \Omega$ is given by the union of two arcs contained respectively in the balls $B_{\lambda}\left(z_{1}^{i}\right)$ and $B_{\lambda}\left(z_{2}^{i}\right)$, and a curve contained in the set of points of $\Omega$ at distance $\lambda-\mu$ from $\Gamma_{i}$, (see Figure 5). Denote by $\alpha_{l}^{i}$ the angle of $A_{i}$ at $y_{l}^{i}, l=1,2$. Remark that

$$
\begin{cases}\alpha_{l}^{i} \rightarrow \frac{\pi}{2}+o_{\lambda}(1) & \text { if } \mu \rightarrow 0 \\ \alpha_{l}^{i} \rightarrow 0 & \text { if } \mu \rightarrow \lambda\end{cases}
$$

where $o_{\lambda}(1) \rightarrow 0$ as $\lambda \rightarrow 0$.

We take as obstacles the functions $\psi_{i}:(\Omega)_{1} \rightarrow \mathbb{R}$ defined as the solutions of the following problem, for $i=1, \ldots, K$,

$$
\begin{cases}\Delta \psi_{i}=0 & \text { in } A_{i} \\ \psi_{i}=f_{i} & \text { on }(\partial \Omega)_{1} \\ \psi_{i}=0 & \text { in } \Omega \backslash A_{i} .\end{cases}
$$

In this section we deal with the solution $\left(u_{1}^{\varepsilon}, \ldots, u_{K}^{\varepsilon}\right)$ of the following obstacle system problem: for $i=1, \ldots, K$,

$$
\begin{cases}u_{i}^{\varepsilon} \geq \psi_{i} & \text { in } \Omega, \\ \Delta u_{i}^{\varepsilon}(x) \leq \frac{1}{\varepsilon^{2}} u_{i}^{\varepsilon}(x) \sum_{j \neq i} H\left(u_{j}^{\varepsilon}\right)(x) & \text { in } \Omega, \\ \Delta u_{i}^{\varepsilon}(x)=\frac{1}{\varepsilon^{2}} u_{i}^{\varepsilon}(x) \sum_{j \neq i} H\left(u_{j}^{\varepsilon}\right)(x) & \text { in }\left\{u_{i}^{\varepsilon}>\psi_{i}\right\} \\ u_{i}^{\varepsilon}=f_{i} & \text { on }(\partial \Omega)_{1} .\end{cases}
$$

In the whole section we make the following assumptions:

$$
\left\{\begin{array}{l}
\varepsilon>0, \\
(8.31) \text { and }(8.32) \text { hold true, } \\
H \text { is either of the form } 2.5 \text { with } p=1 \text {, or } 2.6 \text { and } 2.7 \text { holds true; } \\
\text { For } i=1, \ldots, K, A_{i} \text { and } \psi_{i} \text { are defined by } 8.33 \text { and } 8.35 \text { respectively. }
\end{array}\right.
$$

Theorem 8.13. Assume (8.37). Then, there exist continuous positive functions $u_{1}^{\varepsilon}, \ldots, u_{K}^{\varepsilon}$, depending on the parameter $\varepsilon$, viscosity solutions of the problem (8.36). In particular

$$
\Delta u_{i}^{\varepsilon}(x)=\frac{1}{\varepsilon^{2}} u_{i}^{\varepsilon}(x) \sum_{j \neq i} H\left(u_{j}^{\varepsilon}\right)(x) \quad \text { in } \Omega \backslash A_{i} .
$$

Moreover, for $i=1, \ldots, K$,

$$
\Delta u_{i}^{\varepsilon} \geq 0 \quad \text { in } \Omega
$$


in the viscosity sense.

Proof. The proof of the existence of a solution $\left(u_{1}^{\varepsilon}, \ldots, u_{K}^{\varepsilon}\right)$ of 8.36 is a slightly modification of the proof of Theorem 4.1 . Here

$$
\Theta=\left\{\left(u_{1}, u_{2}, \ldots, u_{K}\right) \mid u_{i}: \Omega \rightarrow \mathbb{R} \text { is continuous, } \psi_{i} \leq u_{i} \leq \phi_{i} \text { in } \Omega, u_{i}=f_{i} \text { on }(\partial \Omega)_{1}\right\} \text {. }
$$

In the set $\Omega \backslash A_{i}$, we have that $u_{i}^{\varepsilon}>0=\psi_{i}$ which implies (8.38). Inequality (8.39) is a consequence of the following facts: in the set $\left\{u_{i}^{\varepsilon}>\psi_{i}\right\}$ we have $\Delta u_{i}^{\varepsilon}=\frac{1}{\varepsilon^{2}} u_{i}^{\varepsilon} \sum_{j \neq i} H\left(u_{j}^{\varepsilon}\right) \geq 0$; in the interior of the set $\left\{u_{i}^{\varepsilon}=\psi_{i}\right\}, \Delta u_{i}^{\varepsilon}=\Delta \psi_{i}=0$; the free boundaries $\partial\left\{u_{i}^{\varepsilon}>\psi_{i}\right\}$ have locally finite $n-1$-Hausdorff measure, see [2].

Theorem 8.14. Assume (8.37). Let $\left(u_{1}^{\varepsilon}, \ldots, u_{K}^{\varepsilon}\right)$ be viscosity solution of the problem 8.36). Then, there exists a subsequence $\left(u_{1}^{\varepsilon_{l}}, \ldots, u_{K}^{\varepsilon_{l}}\right)$ and continuous functions $\left(u_{1}, \ldots, u_{K}\right)$ defined on $\bar{\Omega}$, such that

$$
\left(u_{1}^{\varepsilon_{l}}, \ldots, u_{K}^{\varepsilon_{l}}\right) \rightarrow\left(u_{1}, \ldots, u_{K}\right) \quad \text { as } l \rightarrow+\infty, \quad \text { a.e. in } \Omega
$$

and the convergence of $u_{i}^{\varepsilon_{l}}$ to $u_{i}$ is locally uniform in the support of $u_{i}$. Moreover, we have:

i) the $u_{i}$ 's are locally Lipschitz continuous in $\Omega$, in particular, there exists $C_{0}>0$ such that, if $d_{\rho}(x, \partial \Omega) \geq r$, then

$$
\left|\nabla u_{i}(x)\right| \leq \frac{C_{0}}{r}
$$

ii) the $u_{i}$ 's have disjoint supports, more precisely:

$$
u_{i} \equiv 0 \quad \text { in the set } \quad\left\{x \in \Omega \mid d_{\rho}\left(x, \operatorname{supp} u_{j}\right) \leq 1\right\} \quad \text { for any } j \neq i .
$$

iii) $\Delta u_{i}=0$ when $u_{i}>0$.

iv) $u_{i} \geq \psi_{i}$ in $\Omega$.

v) $u_{i}=f_{i}$ on $\partial \Omega$.

Proof. The convergence theorem is again a consequence of Lemma 5.3, Corollary 5.4 and Lemma 5.5 which hold true with $\operatorname{supp} f_{i}$ and $\operatorname{supp} f_{j}$ replaced respectively by supp $\psi_{i}=A_{i}$ and 
$\operatorname{supp} \psi_{j}=A_{j}$ (in Lemma 5.3 and Corollary 5.4), and $\bar{\Gamma}_{j}^{\sigma}$ defined as the set $\left\{\psi_{j} \geq \sigma\right\}$ (in Lemma 5.5). Estimates (5.7) of Corollary 5.4 in particular imply (8.40). Property (iv) is an immediate consequence of $u_{i}^{\varepsilon} \geq \psi_{i}$ in $\Omega$. Finally, (v) is implied by the fact that $\psi_{i} \leq u_{i}^{\varepsilon} \leq \phi_{i}$ in $\Omega$, and $\phi_{i}=\psi_{i}=f_{i}$ on $\partial \Omega$, where $\phi_{i}$ is given by (4.1).

As proven in Corollary 6.2, one can show that the free boundaries satisfy the exterior $\rho$-ball condition with radius 1 , that they have finite 1-Hausdorff dimensional measure and that the distance between the support of two different functions is precisely one. We are now going to prove that, if $\lambda-\mu$ is small enough, then any solution of the obstacle problem (8.36) never touches the obstacles inside the domain $\Omega$. To this aim, we first need the following lemma:

Lemma 8.15. Assume (8.37). Then, there exists $c>0$ such that, for $i=1, \ldots, K$, we have

$$
\frac{\partial \psi_{i}}{\partial \nu_{i}}(x) \leq-\frac{c}{\lambda-\mu} \quad \text { for any } x \in \partial A_{i} \cap \Omega,
$$

where $\nu_{i}$ is the exterior normal vector to the set $A_{i}$.

Proof. Fix any point $x_{0} \in \partial A_{i} \cap \Omega$. Then, by definition of $A_{i}$, there exists a point $z \in \Omega^{c}$ such that $d(z, \partial \Omega)=\mu, B_{\lambda}(z) \cap \Omega \subset A_{i}$ and $x_{0} \in \partial B_{\lambda}(z)$. Consider now the ring $\{x|\mu<| x-z \mid<\lambda\}$ and the barrier function $\phi$ solution of

$$
\begin{cases}\Delta \phi=0 & \text { in }\{x|\mu<| x-z \mid<\lambda\} \\ \phi=1 & \text { on } \partial B_{\mu}(z) \\ \phi=0 & \text { on } \partial B_{\lambda}(z) .\end{cases}
$$

The function $\psi_{i}$ is harmonic in $B_{\lambda}(z) \cap \Omega, \psi_{i} \geq 0=\phi$ on $\partial B_{\lambda}(z) \cap \Omega$ and $\psi_{i}=1 \geq \phi$ on $\partial \Omega \cap B_{\lambda}(z)$. Therefore by the comparison principle, we have that $\psi_{i}(x) \geq \phi(x)$ for any $x \in B_{\lambda}(z) \cap \Omega$, and this implies (8.41) at $x=x_{0}$.

Theorem 8.16. Assume (8.37). Let $\left(u_{1}, \ldots, u_{K}\right)$ be the limit of a converging subsequence of $\left(u_{1}^{\varepsilon}, \ldots, u_{K}^{\varepsilon}\right)$, solution of 8.36. Set $a:=\lambda-\mu$. Then, there exists $a_{0}>0$ such that for any $a<a_{0}$, we have, for $i=1, \ldots, K$,

$$
u_{i}>\psi_{i} \quad \text { in } \bar{A}_{i} \cap \Omega \text {. }
$$


Proof. In order to prove $(8.42)$, it is enough to show that

$$
u_{i}(x)>\psi_{i}(x), \quad \text { for any } x \in \partial A_{i} \cap \Omega .
$$

Indeed, if 8.43 holds true, since by 8.35 and Theorem 8.14, both $u_{i}$ and $\psi_{i}$ are harmonic in $A_{i}$, the strong maximum principle implies $u_{i}>\psi_{i}$ in $A_{i}$. This and 8.43 give 8.42 . Suppose by contradiction that there exists a point $x_{0} \in \partial A_{i} \cap \Omega$ such that $u_{i}\left(x_{0}\right)=\psi_{i}\left(x_{0}\right)=0$. Then, by 8.41 , we have that

$$
\frac{\partial u_{i}}{\partial \nu_{i}}\left(x_{0}\right) \leq \frac{\partial \psi_{i}}{\partial \nu_{i}}\left(x_{0}\right) \leq-\frac{c}{\lambda-\mu}=-\frac{c}{a}
$$

Assumptions (8.31) imply that if the angles $\alpha_{l}^{i}$ of $A_{i}$ at $y_{l}^{i}, l=1,2$, are small enough, the sets defined by

$$
\Sigma_{i}=\left\{y: y=x+\nu_{i}(x), x \in \partial A_{i} \cap \Omega\right\}
$$

and

$$
\Sigma_{i}^{-}=\left\{y: y=x+t \nu_{i}(x), x \in \partial A_{i} \cap \Omega, 0<t<1\right\}
$$

are compactly supported in $\Omega$ and

$$
d_{\rho}\left(x_{0}, \operatorname{supp} \psi_{j}\right)>1 \quad \text { for any } j \neq i \text {. }
$$

Therefore, by 8.34, we can choose $a$ so small that 8.45 holds true. Moreover, from 8.45, there exists a small $\sigma>0$ such that $\mathcal{B}_{1+\sigma}\left(x_{0}\right) \cap \operatorname{supp} \psi_{j}=\emptyset, j \neq i$, and from (8.36), we know that

$$
\Delta u_{j}^{\varepsilon} \geq \frac{1}{\varepsilon^{2}} u_{j}^{\varepsilon} H\left(u_{i}^{\varepsilon}\right) \quad \text { in } \mathcal{B}_{1+\sigma}\left(x_{0}\right)
$$

(consider $u_{j}^{\varepsilon}$ extended by zero if the ball falls out of $\Omega$ ). When $H$ is defined as in (2.5) with $p=1$, arguing as in 8.27 in proof of Theorem 8.10 we obtain that

$$
\sum_{j \neq i} \int_{\left(D_{\sigma}\left(x_{0}\right)\right)_{1}} \Delta u_{j} \geq \int_{D_{\sigma}\left(x_{0}\right)} \Delta u_{i} .
$$

Now, since $u_{i} \geq \psi_{i}>0$ in $A_{i}$ and $u_{i}\left(x_{0}\right)=0$, the point $x_{0}$ belongs to $\partial\left\{u_{i}>0\right\} \cap \partial A_{i} \cap \Omega$. Since $\partial A_{i} \cap \Omega$ has an interior tangent ball and $\partial\left\{u_{i}>0\right\}$ has a exterior tangent ball, we know 
that $x_{0}$ is a regular point. Since the set of regular points is an open set, see Lemma 8.9, for $\sigma$ small enough we have

$$
\int_{D_{\sigma}\left(x_{0}\right)} \Delta u_{i} \geq-\int_{\partial\left\{u_{i}>0\right\} \cap D_{\sigma}\left(x_{0}\right)} \frac{\partial u_{i}}{\partial \nu_{i}} d \mathcal{H}
$$

where $\nu_{i}$ is still the exterior normal vector to $A_{i}$. On another hand, if $y_{0}$ is the point that realizes the distance one with $x_{0}$, assume w.l.o.g. that $y_{0} \in \partial \operatorname{supp} u_{j}, y_{0}$ has to be in $\Sigma_{i}$ and $y_{0}$ has to be a regular point. Then, for $\rho$ small enough such that $\partial\left\{u_{j}>0\right\} \cap B_{\rho}\left(y_{0}\right)$ is $C^{1}$ we have

$$
\int_{B_{\rho}\left(y_{0}\right)} \Delta u_{j}=-\int_{\partial\left\{u_{j}>0\right\} \cap B_{\rho}\left(y_{0}\right)} \frac{\partial u_{j}}{\partial \nu_{j}} d \mathcal{H} .
$$

Now, using the fact that for $\sigma$ small enough such that $\rho>c \sigma, \operatorname{supp} u_{j} \cap\left(D_{\sigma}\left(x_{0}\right)\right)_{1} \subset \mathcal{B}_{c \sigma}\left(y_{0}\right)$, we have

$$
\int_{B_{c \sigma}\left(y_{0}\right)} \Delta u_{j} \geq \int_{\left(D_{\sigma}\left(x_{0}\right)\right)_{1}} \Delta u_{i}
$$

Putting all together, dividing (8.46) and 8.47) respectively by $\mathcal{H}\left(\partial\left\{u_{i}>0\right\} \cap D_{\sigma}\left(x_{0}\right)\right)$ and $\mathcal{H}\left(\partial\left\{u_{j}>0\right\} \cap B_{c \sigma}\left(y_{0}\right)\right)$, and passing to the limit when $\sigma \rightarrow 0$ we obtain

$$
-\frac{\partial u_{j}}{\partial \nu_{j}}\left(y_{0}\right) \geq-c \frac{\partial u_{i}}{\partial \nu_{i}}\left(x_{0}\right) \geq \frac{\tilde{c}}{a}
$$

We are now going to show that 8.48 yields a contradiction. Indeed, the point $y_{0}$ realizes its distance from the set $\left\{u_{i}>0\right\}$ at $x_{0}$, therefore the ball $\mathcal{B}_{1}\left(y_{0}\right)$ is tangent to $\left\{u_{i}>0\right\}$ at $x_{0}$. Moreover, since $A_{i} \subset\left\{u_{i}>0\right\}$, the ball $\mathcal{B}_{1}\left(y_{0}\right)$ is tangent to $A_{i}$ at $x_{0}$. On the other hand, for $a$ small enough, by assumption (8.31), $\mathcal{B}_{1}\left(y_{0}\right)$ is contained in $\Omega$. In particular, the $\rho$-distance of $y_{0}$ from $\partial \Omega$ is greater than 1 . Therefore, from $(8.40)$, we infer that $\left|\nabla u_{j}\left(y_{0}\right)\right| \leq C_{0}$, which is in contradiction with 8.48 for $a$ small enough.

When $H$ is defined as in (2.6), we argue as in case (b) in the proof of Theorem 7.1 and similarly, we get a contradiction for a small enough. 
Corollary 8.17. Under the assumptions of Theorem 8.16, if a $<a_{0}$ then $\left(u_{1}^{\varepsilon}, \ldots, u_{K}^{\varepsilon}\right)$ is solution of the following problem

$$
\begin{cases}u_{i}^{\varepsilon} \geq \psi_{i} & \text { in } \Omega, \\ \Delta u_{i}^{\varepsilon}(x)=\frac{1}{\varepsilon^{2}} u_{i}^{\varepsilon}(x) \sum_{j \neq i} H\left(u_{j}^{\varepsilon}\right)(x) & \text { in } \Omega \\ u_{i}^{\varepsilon}=f_{i} & \text { on }(\partial \Omega)_{1} .\end{cases}
$$

In particular, $\left(u_{1}^{\varepsilon}, \ldots, u_{K}^{\varepsilon}\right)$ is solution of (2.4).

We are now ready to show that free boundaries are Lipschitz.

Theorem 8.18. Let $\left(u_{1}^{\varepsilon}, \ldots, u_{K}^{\varepsilon}\right)$ be the solution of (2.4) given by Corollary 8.17, Let $\left(u_{1}, \ldots, u_{K}\right)$ be the limit as $\varepsilon \rightarrow 0$ of a converging subsequence, then the free boundaries $\partial\left\{u_{i}>0\right\}$, $i=1, \ldots, K$, are Lipschitz curves of the plane.

Proof. By contradiction let's assume that the free boundaries are not Lipschitz. This would imply that there exists at least one singular point with asymptotic cone with zero opening.

Let $x_{0}$ be an interior singular point with asymptotic cone with zero angle. W.l.o.g. suppose $x_{0} \in \partial\left\{u_{1}>0\right\}$. Let $e_{1}$ be the line perpendicular to the cone axis and passing through $x_{0}$, in which we choose an orientation such that the cone is below the axis $e_{1}$. As we proved in Theorem 8.10 and Corollary 8.12 there exist $y_{0}$ and $y_{1}$, with $y_{0}, y_{1} \in \cup_{j \neq 1} \partial\left\{u_{j}>0\right\}$ singular points at distance one from $x_{0}$ with asymptotic cones with zero opening. Also, by Theorem 7.1 for any regular point $x \in \partial\left\{u_{1}>0\right\} \cap B_{1}\left(x_{0}\right)$ there exists a correspondent $y \in \cup_{j \neq 1} \partial\left\{u_{j}>0\right\}$ such that

$$
y=x+\nu(x)
$$

with $\nu(x)$ the external normal vector to $\partial\left\{u_{1}>0\right\}$ at $x$. Observe that $y_{0}, y_{1}$ must lie on $e_{1}$. In fact, let $x_{n}^{l} \in \partial\left\{u_{1}>0\right\}$ be regular points converging to $x_{0}, x_{n}^{l} \rightarrow x_{0}$ as $n \rightarrow+\infty$, from the left side of the cone axis and let $x_{n}^{r} \in \partial\left\{u_{1}>0\right\}$ be the regular points such that $x_{n}^{r} \rightarrow x_{0}$ as $n \rightarrow+\infty$, from the right side of the cone axis. Then, the limit of the normal vectors $\nu\left(x_{n}^{l}\right) \rightarrow \nu^{l}$ and $\nu\left(x_{n}^{r}\right) \rightarrow \nu^{r}$, are both on the direction $e_{1}$ since they are orthogonal to the cone axis. Let 
$y_{0}$ and $y_{1}$ be w.l.o.g. the points defined by

$$
y_{0}=x_{0}+\nu^{l} \quad y_{1}=x_{0}+\nu^{r} .
$$

So we have to have three singular points at distance one, all on the line $e_{1}$. Repeating the same argument and using $y_{1}$ as the reference singular point now, we conclude that there must exist another singular point, $y_{2}$, with 0 opening cone, at distance one from $y_{1}$ and also on the axis $e_{1}$. Iterating, we will be able to proceed until the prescribed boundary of the domain stops us from finding the next point. We will have all singular points with cone with zero opening aligned on the axis $e_{1}$, until we reach the boundary $\partial \Omega$ and we cannot proceed with this process, i.e., until we cannot obtain the next point aligned in the direction of $e_{1}$ which implies that $\partial \Omega$ crosses the axis $e_{1}$ and the distance of $y_{k}$ to the boundary of $\Omega$ along $e_{1}$ is less or equal than 1 .

Now, there are two cases: either $y_{k} \in \partial \Omega$ or $y_{k} \in \Omega$. If $y_{k} \in \partial \Omega$ assume w.l.o.g. that $y_{k} \in \partial\left\{u_{1}>0\right\}$. Since $u_{1} \geq \psi_{1}$ we have $A_{1} \subset\left\{u_{1}>0\right\}$ and that $y_{k}$ must coincide with one of the points $y_{l}^{1}, l=1,2$, endpoints of the curve $\Gamma_{1}$. Indeed, by the forth assumption in 8.32 , no points of $\partial\left\{u_{1}>0\right\}$ are on $\partial \Omega$ between the curves $\Gamma_{1}$ and $\Gamma_{2}$, and $\Gamma_{1}$ and $\Gamma_{K}$. Assume w.l.o.g. that $y_{k}=y_{1}^{1}$. Let $\theta$ be the angle of $\partial\left\{u_{1}>0\right\}$ at $y_{1}^{1}$. Then, from (8.14) of Theorem 8.10 applied to $y_{k}=y_{1}^{1}$ and $y_{0}=y_{k-1}$, we get $\theta=0$. On the other hand, since $A_{1} \subset\left\{u_{1}>0\right\}$ then $\theta \geq \alpha_{1}^{1}>0$, where $\alpha_{1}^{1}$ is the angle of $A_{1}$ at $y_{1}^{1}$. We have obtained a contradiction. Suppose now that $y_{k}$ is an interior point. Again, assume w.l.o.g. that $y_{k} \in \partial\left\{u_{1}>0\right\}$. Let $z_{k} \in \partial \Omega$ be the closest point to $y_{k}$ in the direction $e_{1}$ and $d\left(y_{k}, z_{k}\right)=l<1$. Recall that by 8.31 there is an exterior tangent ball at $z_{k}, B_{1+\eta}$, so once the axis $e_{1}$ is crossed, $\Omega$ will remain outside of the tangent ball at $z_{k}$ and so $\partial \Omega$ will not cross again $e_{1}$ in $\overline{\mathcal{B}}_{1}\left(y_{k}\right)$. We know that $z_{k}$ cannot belong to $\partial\left\{u_{j}>0\right\}$ since it does not respect the distance one and also $A_{j} \subset\left\{u_{j}>0\right\}$. And by Theorem 7.1 for any point on the free boundary there exists a correspondent point at distance one belonging to the support of another function. Taking in account the previous case, the only 


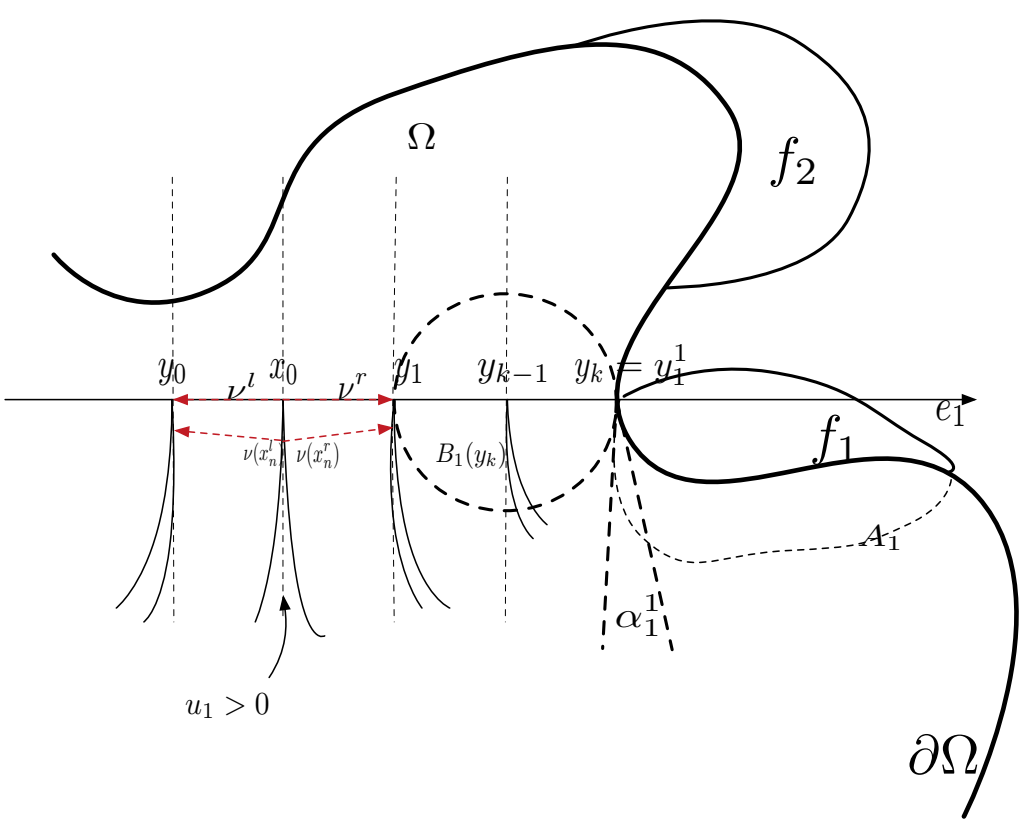

Figure 6. Contradiction in the case $y_{k} \in \partial \Omega$

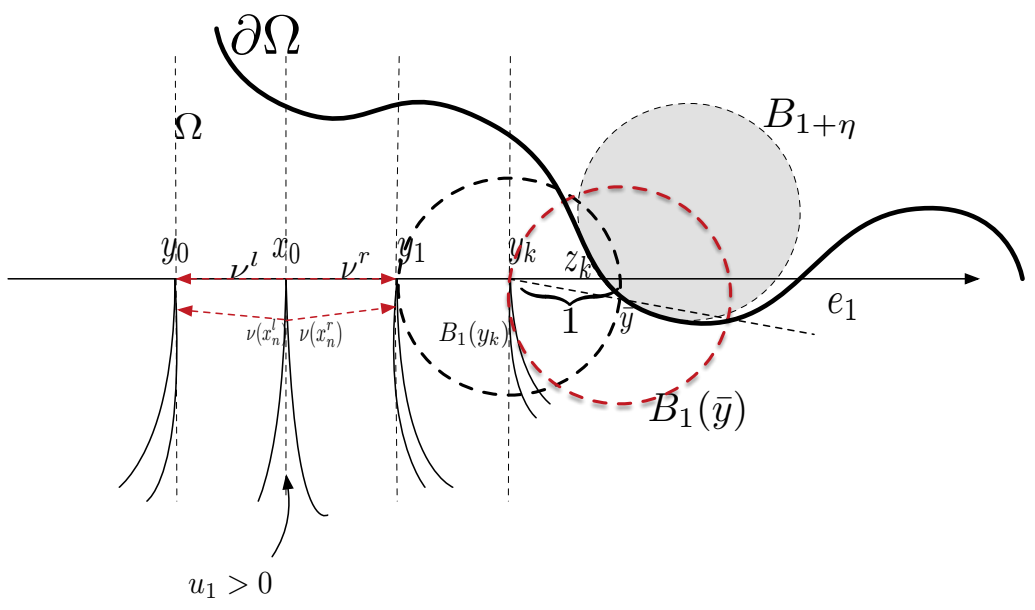

Figure 7. Contradiction in the case $y_{k} \in \Omega$ 
option is that the point that realizes the distance from $y_{k}, \bar{y}$, belongs to $B_{1}\left(y_{k}\right)$ and it must be such that the angle between $e_{1}$ and the line that contains both $y_{k}$ and $\bar{y}$ is strictly positive, see Figure 7. Therefore, we must conclude that $B_{1}(\bar{y}) \cap\left\{u_{1}>0\right\} \neq \emptyset$.

We have obtained a contradiction. We conclude that the free boundaries cannot have a zero angle at a singular point, therefore they are Lispschitz curves of the plane.

\section{A Relation Between the nORMAL DERIVATIVES AT THE FREE BOUNDARY}

In this section we restrict ourself to the following case:

$$
\left\{\begin{array}{l}
K=2 \\
H \text { defined like in }(2.5), \text { with } \\
p=1, \varphi \equiv 1 \text { and } \rho \text { the Euclidian norm. }
\end{array}\right.
$$

Therefore, the system (2.4) becomes

$$
\begin{aligned}
& \Delta u_{1}^{\varepsilon}(x)=\frac{1}{\varepsilon^{2}} u_{1}^{\varepsilon}(x) \int_{B_{1}(x)} u_{2}^{\varepsilon}(y) \mathrm{d} y \quad \text { in } \Omega, \\
& \Delta u_{2}^{\varepsilon}(x)=\frac{1}{\varepsilon^{2}} u_{2}^{\varepsilon}(x) \int_{B_{1}(x)} u_{1}^{\varepsilon}(y) \mathrm{d} y \quad \text { in } \Omega,
\end{aligned}
$$

where we denote by $B_{1}(x)$ the Euclidian ball of radius 1 centered at $x$. Let $\left(u_{1}, u_{2}\right)$ be the limit functions of a converging subsequence that we still denote $\left(u_{1}^{\varepsilon}, u_{2}^{\varepsilon}\right)$ and for $i=1,2$ let

$$
S_{i}:=\left\{u_{i}>0\right\}
$$

From Section 7 we know that the $u_{i}$ 's have disjoint support and that there is a strip of width exactly one that separates $S_{1}$ and $S_{2}$. Moreover, Corollary 6.2 guarantees that at any point of the boundary of the two sets, the principal curvatures are less or equal 1 . For $i=1,2$, let $x_{i} \in \partial S_{i}$ be such that $x_{1}$ is at distance 1 from $x_{2}, \partial S_{i}$ is of class $C^{2}$ in a neighborhood of $x_{i}$, and all the principal curvatures of $\partial S_{i}$ at $x_{i}$ are strictly less than 1 . Without loss of generality we can assume $x_{1}=0$ and $x_{2}=e_{n}$, where $e_{n}=(0, \ldots, 1)$. Let us denote by $u_{\nu}^{1}(0)$ and $u_{\nu}^{2}\left(e_{n}\right)$ the exterior normal derivatives of $u_{1}$ and $u_{2}$ respectively at 0 and $e_{n}$. Note that the two normals have opposite direction. We want to deduce a relation between $u_{\nu}^{1}(0)$ and $u_{\nu}^{2}\left(e_{n}\right)$. Let us start by recalling some basic properties about the level surfaces of the distance function to a set. 


\subsection{Level surfaces of the distance function to a set. Some basic Properties. Consider}

a bounded open set $S$ and its boundary $\partial S$, of the class $C^{2}$. Let $\varkappa_{i}(x)$ be the principal curvatures of $\partial S$ at $x$ (outward is the positive direction). Assume that for any point $x \in \partial S$ there exists a tangent ball $B_{R}(z)$ to $\partial S$ at $x$ such that $B_{R}(z) \subset S^{c}$. In particular the principle curvatures satisfy $\varkappa_{i}(x) \leq 1 / R, i=1, \ldots, n-1$. Then:

a) the distance function to $S, d_{S}(x)=d(x, \bar{S})$, is defined and is $C^{2}$ as long as

$$
0<d_{S}(x)<R
$$

In the following lemma, which may be known in the literature, we provide a proof of the $C^{1,1}$-regularity for a more general set, which is not necessary $C^{2}$, it may have edges as well, but it has the property that for any tangent ball there exists a "clean area", in the sense explained below. For the $C^{2}$-regularity in the case of $C^{2}$-boundaries, see for instance Theorem 14.16 in $[23]$.

Given a bounded closed set $F$, we say that $\Pi$ is a supporting hyperplane at $x \in \partial F$, if $x \in \Pi$ and there exists a ball $B \subset F^{c}$ such that $B$ is tangent to $\Pi$ at $x$.

Lemma 9.1. Let $F$ be a bounded closed set. Assume that there exists $R>0$ such that, for any $x \in \partial F$ and any supporting hyperplane $\Pi$ at $x$, there is a ball $B_{R}(z)$ tangent to $\Pi$ at $x$ such that $B_{R}(z) \subset F^{c}$. Let us denote by $d_{F}(x)=d(x, F)$ the distance function from $F$. Then $d_{F}$ is of class $C^{1,1}$ in the set $\left\{0<d_{F}<R\right\}$.

Proof. Let $y_{0} \in\left\{0<d_{F}<R\right\}$. To prove that $d_{F}$ is of class $C^{1,1}$ at $y_{0}$, we show that there are smooth functions whose graphs are tangent from below and above the graph of $d_{F}$ at $\left(y_{0}, d_{F}\left(y_{0}\right)\right)$. As proven in Lemma 6.3), the distance function from a closed bounded set has always a smooth tangent function from above. Indeed, let $x \in \partial F$ be a point where $y_{0}$ realizes the distance from $F$. Assume, without loss of generality, that $x=0$. Then $d\left(y_{0}, 0\right)=\left|y_{0}\right|=d_{F}\left(y_{0}\right)$. Moreover, the ball $B_{\left|y_{0}\right|}\left(y_{0}\right)$ is contained in $F^{c}$ and tangent to $F$ at 0 . For any $y \in B_{\left|y_{0}\right|}\left(y_{0}\right)$, we have that $d_{F}(y) \leq d(y, 0)=|y|$. 
Therefore the cone, graph of the function $y \rightarrow|y|$ (which is smooth at $y_{0} \neq 0$ ) is tangent from above to the graph of $d_{F}$ at $\left(y_{0}, d_{F}\left(y_{0}\right)\right)$.

Next, we prove the existence of a smooth function tangent from below. Note that the tangent line to $B_{\left|y_{0}\right|}\left(y_{0}\right)$ at 0 is a supporting hyperplane to $F$ at 0 . Therefore, there exists a ball $B_{R}(z)$ tangent to $F$ at 0 such that $B_{R}(z) \subset F^{c}$. We must have $z=R y_{0} /\left|y_{0}\right|$. Moreover, since $B_{R}\left(R \frac{y_{0}}{\left|y_{0}\right|}\right) \subset F^{c}$, for any $y \in B_{R}\left(R \frac{y_{0}}{\left|y_{0}\right|}\right) \cap\left\{0<d_{F}<R\right\}$, we have that

$$
d_{F}(y) \geq d\left(y, \partial B_{R}\left(R \frac{y_{0}}{\left|y_{0}\right|}\right)\right)=R-d\left(y, R \frac{y_{0}}{\left|y_{0}\right|}\right)
$$

and $d_{F}\left(y_{0}\right)=\left|y_{0}\right|=R-d\left(y_{0}, R \frac{y_{0}}{\left|y_{0}\right|}\right)$. That is to say, the cone, graph of the function $y \rightarrow R-d\left(y, R \frac{y_{0}}{\left|y_{0}\right|}\right)$ is tangent by below to the graph of $d_{F}$ at $\left(y_{0}, d_{F}\left(y_{0}\right)\right)$. We conclude that $d_{F}$ is $C^{1,1}$ at $y_{0}$.

Let $S(k)$ denote the surface that is at distance $k$ from $S$

$$
S(k):=\left\{x: d_{S}(x)=k\right\}
$$

then, for $k<1+\varepsilon$ and $x \in S(k)$, there is a unique point $x_{0} \in S(0)$, such that $x=x_{0}+k \nu\left(x_{0}\right)$ where $\nu\left(x_{0}\right)$ is the unit normal vector at $x_{0}$ in the positive direction. More precisely, if we denote $K:=\max \left\{\left|\varkappa_{i}(x)\right|: 1 \leq i \leq n-1, x \in \partial S\right\}$ and $f(x, t):=$ $x+t \nu(x)$, then $f$ is a diffeomorphism between $\partial S \times(-k, k)$ and the neighborhood of $\partial S, N_{k}(S)=\{x+t \nu(x): x \in \partial S,|t|<k\}$ with $k<\frac{1}{K}$.

b) for all $x_{0} \in \partial S$ if we consider the linear transformation $x_{t}=x_{0}+t \nu\left(x_{0}\right)$ we obtain $S(t)$. Hence, since the tangent plane for each $S(t)$ is always perpendicular to $\nu\left(x_{0}\right)$, the eigenvectors of the principal curvatures remain constant along the trajectories of $d_{S}$, for $d_{S}<1+\varepsilon$.

c) the curvatures of $S(k)$ satisfy, see Figure 8

$$
\varkappa_{i}\left(x_{0}+k \nu\left(x_{0}\right)\right)=\frac{1}{\frac{1}{\varkappa_{i}\left(x_{0}\right)}-k}=\frac{\varkappa_{i}\left(x_{0}\right)}{1-\varkappa_{i}\left(x_{0}\right) k}, \quad i=1, \ldots, n-1, \quad k<1+\varepsilon
$$




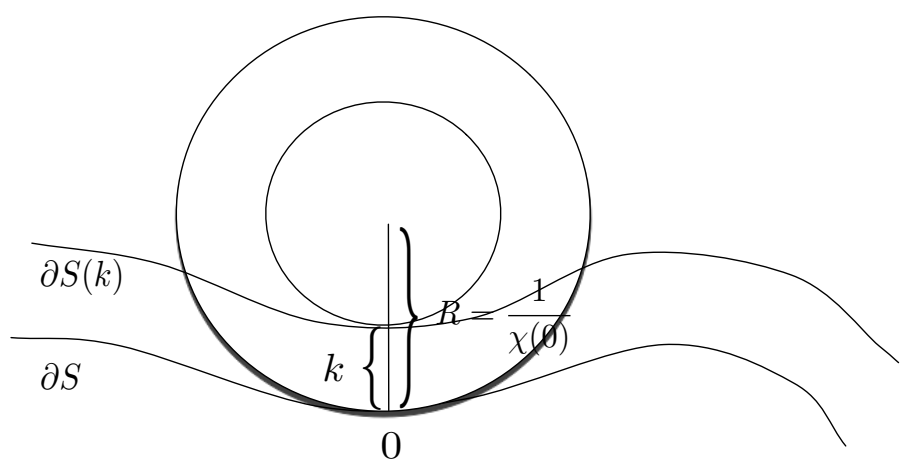

FiguRE 8. Curvatures relation

for $x_{0} \in \partial S$.

d) for $x_{0} \in \partial S$, the ball $B_{1}\left(x_{0}\right)$ touches $S(1)$ at the point $x_{0}+\nu\left(x_{0}\right)$, where $\nu$ is the outward normal. Moreover, it separates quadratically from $S(1)$, that is, for any small $r>0$ and for any $x \in B_{r}\left(x_{0}+\nu\left(x_{0}\right)\right) \cap \partial B_{1}\left(x_{0}\right)$, we have that $d(x, S(1)) \leq C r^{2}$, for some $C>0$.

9.2. Free boundary condition. Following Subsection 9.1, we denote by $\varkappa_{i}(0)$ the principal curvatures of $\partial S_{1}$ at 0 where outward is the positive direction and by $\varkappa_{i}\left(e_{n}\right)=\frac{\varkappa_{i}(0)}{1-\varkappa_{i}(0)}$, the principal curvatures of $\partial S_{2}$ at $e_{n}$. Remark that since the normal vectors to $S_{1}$ and $S_{2}$ respectively at 0 and $e_{n}$, have opposite directions, for $\varkappa_{i}\left(e_{n}\right)$ the inner direction of $S_{2}$ is the positive one. The main result of this section is the following:

Theorem 9.2. Assume (9.1). Let $0 \in \partial S_{1}$ and $e_{n} \in \partial S_{2}$. Assume that $\partial S_{1}$ is of class $C^{2}$ in $B_{4 h_{0}}(0)$ and that the principal curvatures satisfy: $\varkappa_{i}(0)<1$ for any $i=1, \ldots, n-1$. Then, we have the following relation:

$$
\frac{u_{\nu}^{1}(0)}{u_{\nu}^{2}\left(e_{n}\right)}=\prod_{\substack{i=1 \\ \varkappa_{i}(0) \neq 0}}^{n-1} \frac{\varkappa_{i}(0)}{\varkappa_{i}\left(e_{n}\right)} \quad \text { if } \varkappa_{i}(0) \neq 0 \text { for some } i=1, \ldots, n-1 \text {, }
$$

and

$$
u_{\nu}^{1}(0)=u_{\nu}^{2}\left(e_{n}\right) \quad \text { if } \varkappa_{i}(0)=0 \text { for any } i=1, \ldots, n-1 \text {. }
$$

In order to prove Theorem 9.2 , we first prove a lemma that relates the mass of the Laplacians of the limit functions across the interfaces. For a point $x$ belonging to a neighborhood of $\partial S_{1}$ 
around 0 , let us denote by $\nu(x)=\nu\left(x_{0}\right)$ the exterior normal vector at $x_{0} \in \partial S_{1}$, where $x_{0}$ is the unique point such that $x=x_{0}+t \nu\left(x_{0}\right)$, for some small $t>0$. From (a) in Subsection 9.1. $\nu(x)$ is well defined.

Lemma 9.3. Under the assumptions of Theorem 9.2, for small $h<h_{0}$, let

$$
D_{h}:=B_{h}(0) \cap\left\{x: d\left(x, \partial S_{1}\right) \leq h^{2}\right\}
$$

and

$$
E_{h}:=\left\{y \in \mathbb{R}^{n} \mid y=x+\nu(x), x \in D_{h}\right\}
$$

Then

$$
\int_{D_{h}} \Delta u_{1}=\int_{E_{h}} \Delta u_{2}
$$

Proof. Remark that the surface $E_{h} \cap \partial S_{2}$ is of class $C^{2}$ for $h$ small enough, being $\varkappa_{i}(0)<1$ for $i=1, \ldots, n-1$, see Subsection 9.1. The Laplacians of the $u_{i}$ 's are positive measures and

$$
\int_{D_{h}} \Delta u_{1}=\lim _{\varepsilon \rightarrow 0} \int_{D_{h}} \Delta u_{1}^{\varepsilon}(x) \mathrm{d} x=\lim _{\varepsilon \rightarrow 0} \frac{1}{\varepsilon^{2}} \int_{D_{h}} \int_{B_{1}(x)} u_{1}^{\varepsilon}(x) u_{2}^{\varepsilon}(y) \mathrm{d} y \mathrm{~d} x,
$$

and

$$
\int_{E_{h}} \Delta u_{2}=\lim _{\varepsilon \rightarrow 0} \int_{E_{h}} \Delta u_{2}^{\varepsilon}(y) \mathrm{d} y=\lim _{\varepsilon \rightarrow 0} \frac{1}{\varepsilon^{2}} \int_{E_{h}} \int_{B_{1}(y)} u_{1}^{\varepsilon}(x) u_{2}^{\varepsilon}(y) \mathrm{d} x \mathrm{~d} y .
$$

Let $s$ be such that $\varepsilon^{\frac{1}{4 \alpha}}<s<h$, where $\alpha$ is given by Lemma 5.3 . We split the set $D_{h}$ in the following way

$$
D_{h}=D_{h, s}^{+} \cup D_{h, s}^{-} \cup D_{h, s},
$$

where

$$
\begin{gathered}
D_{h, s}^{+}:=\left\{x \in D_{h} \mid d\left(x, \partial S_{1}\right)>s^{2} \text { and } u_{1}(x)>0\right\}, \\
D_{h, s}^{-}:=\left\{x \in D_{h} \mid d\left(x, \partial S_{1}\right)>s^{2} \text { and } u_{1}(x)=0\right\}, \\
D_{h, s}:=\left\{x \in D_{h} \mid d\left(x, \partial S_{1}\right) \leq s^{2}\right\} .
\end{gathered}
$$

Similarly

$$
E_{h}=E_{h, s}^{+} \cup E_{h, s}^{-} \cup E_{h, s},
$$


where

$$
\begin{gathered}
E_{h, s}^{+}:=\left\{x \in E_{h} \mid d\left(x, \partial S_{2}\right)>s^{2} \text { and } u_{2}(x)>0\right\}, \\
E_{h, s}^{-}:=\left\{x \in E_{h} \mid d\left(x, \partial S_{2}\right)>s^{2} \text { and } u_{2}(x)=0\right\}, \\
E_{h, s}:=\left\{x \in E_{h} \mid d\left(x, \partial S_{2}\right) \leq s^{2}\right\},
\end{gathered}
$$

see Figure 9. Since $\partial S_{1}$ is a smooth surface around 0 , and $\Delta u_{1}=0$ in $S_{1}$, we have that $u_{1}$ grows linearly away from the boundary in a neighborhood of 0 . This and the uniform convergence of $u_{1}^{\varepsilon}$ to $u_{1}$, imply that there exists $c>0$ such that $u_{1}^{\varepsilon}(x)>c s^{2}$, for any $x \in D_{h, s}^{+}$for $\varepsilon$ small enough. Then, by Lemma $5.3, u_{2}^{\varepsilon}(y) \leq a e^{-\frac{b\left(c s^{2}\right)^{\alpha}}{\varepsilon}},\left(a, b\right.$ positive constants), for $y \in B_{1}(x)$ and any $x \in D_{h, s}^{+}$. In an analogous way, if $y \in E_{h, s}^{+}$, we know that for $\varepsilon$ small enough $u_{2}^{\varepsilon}(y)>c s^{2}$ and by Lemma 5.3. $u_{1}^{\varepsilon}(x) \leq a e^{-\frac{b\left(c s^{2}\right)^{\alpha}}{\varepsilon}}$ for $x \in B_{1}(y)$. Since we have chosen $s$ such that $s^{2 \alpha}>\varepsilon^{\frac{1}{2}}$, we have that $u_{2}^{\varepsilon}(y)=o\left(\varepsilon^{2}\right)$ uniformly in $y$, for any $y \in \cup_{x \in D_{h, s}^{+}} B_{1}(x)$ and $u_{1}^{\varepsilon}(x)=o\left(\varepsilon^{2}\right)$ uniformly in $x$, for any $x \in \cup_{y \in E_{h, s}^{+}} B_{1}(y)$. Remark that

$$
D_{h, s}^{-} \subset \cup_{y \in E_{h, s}^{+}} B_{1}(y)
$$

Therefore we have

$$
\begin{aligned}
\frac{1}{\varepsilon^{2}} \int_{x \in D_{h}} \int_{y \in B_{1}(x)} u_{1}^{\varepsilon}(x) u_{2}^{\varepsilon}(y) \mathrm{d} y \mathrm{~d} x & =\frac{1}{\varepsilon^{2}} \int_{x \in D_{h, s}^{+}} \int_{y \in B_{1}(x)} u_{1}^{\varepsilon}(x) \underbrace{u_{2}^{\varepsilon}(y)}_{\text {negligible }} \mathrm{d} y \mathrm{~d} x \\
& +\frac{1}{\varepsilon^{2}} \int_{x \in D_{h, s}} \int_{y \in B_{1}(x)} u_{1}^{\varepsilon}(x) u_{2}^{\varepsilon}(y) \mathrm{d} y \mathrm{~d} x \\
& +\frac{1}{\varepsilon^{2}} \int_{x \in D_{h, s}^{-}} \int_{y \in B_{1}(x)} \underbrace{u_{1}^{\varepsilon}(x)}_{\text {negligible }} u_{2}^{\varepsilon}(y) \mathrm{d} y \mathrm{~d} x \\
& =\frac{1}{\varepsilon^{2}} \int_{x \in D_{h, s}} \int_{y \in B_{1}(x)} u_{1}^{\varepsilon}(x) u_{2}^{\varepsilon}(y) \mathrm{d} y \mathrm{~d} x+o(1) .
\end{aligned}
$$

Analogously

$$
\frac{1}{\varepsilon^{2}} \int_{y \in E_{h}} \int_{x \in B_{1}(y)} u_{1}^{\varepsilon}(x) u_{2}^{\varepsilon}(y) \mathrm{d} x \mathrm{~d} y=\frac{1}{\varepsilon^{2}} \int_{E_{h, s}} \int_{B_{1}(y)} u_{1}^{\varepsilon}(x) u_{2}^{\varepsilon}(y) \mathrm{d} x \mathrm{~d} y+o(1) .
$$

Next, for fixed $x \in D_{h, s}$, we have

$$
B_{1}(x) \cap\left\{y \mid d\left(y, \partial S_{2}\right)>s^{2}\right\} \subset B_{1+h}(0) \cap\left\{y \mid d\left(y, \partial S_{2}\right)>s^{2}\right\} \cap\left\{u_{2} \equiv 0\right\} .
$$




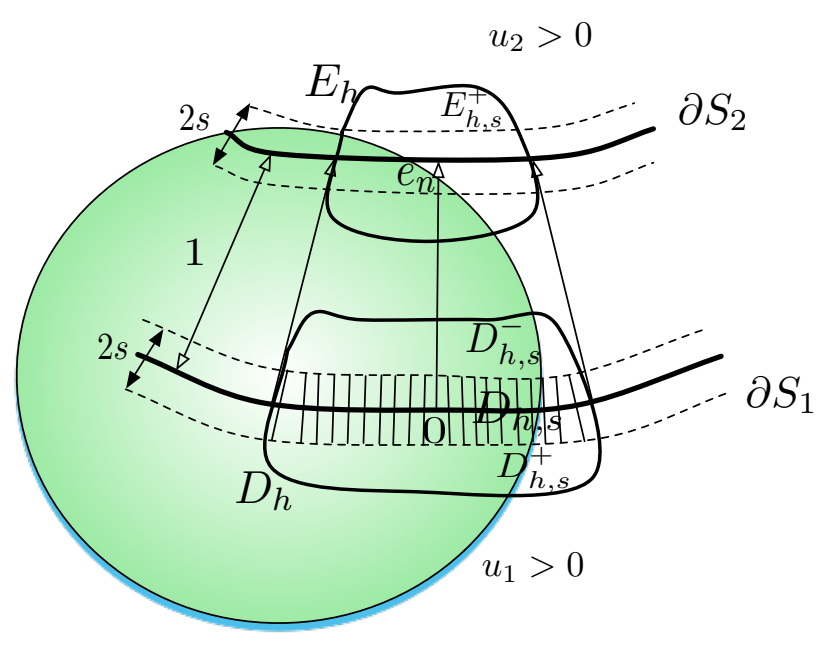

Figure 9. Relation between the mass of the Laplacians

Therefore for any $y \in B_{1}(x) \cap\left\{y \mid d\left(y, \partial S_{2}\right)>s^{2}\right\}$, the ball $B_{1}(y)$ enters in $S_{1} \cap B_{2 h}(0)$ at distance at least $s^{2}$ from $\partial S_{1}$. Since $\partial S_{1} \cap B_{4 h}(0)$ is of class $C^{2}, u_{1}$ has linear growth away from the boundary in $\partial S_{1} \cap B_{2 h}(0)$ and therefore there exists a point in $B_{1}(y)$ where $u_{1} \geq c s^{2}$ for some $c>0$. Like before, Lemma 5.3 implies that $u_{2}^{\varepsilon}(y)=o\left(\varepsilon^{2}\right)$. We infer that

$\frac{1}{\varepsilon^{2}} \int_{x \in D_{h, s}} \int_{y \in B_{1}(x)} u_{1}^{\varepsilon}(x) u_{2}^{\varepsilon}(y) \mathrm{d} y \mathrm{~d} x=\frac{1}{\varepsilon^{2}} \int_{x \in D_{h, s}} \int_{y \in B_{1}(x) \cap\left\{y \mid d\left(y, \partial S_{2}\right) \leq s^{2}\right\}} u_{1}^{\varepsilon}(x) u_{2}^{\varepsilon}(y) \mathrm{d} y \mathrm{~d} x+o(1)$.

Finally, remark that (d) of Subsection 9.1 implies that for $x \in D_{h, s}$

$$
B_{1}(x) \cap\left\{y \mid d\left(y, \partial S_{2}\right) \leq s^{2}\right\} \subset E_{h+c s, s}
$$

for some $c>0$. From 9.2, 9.3, 9.4 and 9.5, we get

$$
\begin{aligned}
\int_{D_{h}} \Delta u_{1}^{\varepsilon}(x) d x & =\frac{1}{\varepsilon^{2}} \int_{x \in D_{h}} \int_{y \in B_{1}(x)} u_{1}^{\varepsilon}(x) u_{2}^{\varepsilon}(y) \mathrm{d} y \mathrm{~d} x \\
& =\frac{1}{\varepsilon^{2}} \int_{x \in D_{h, s}} \int_{y \in B_{1}(x) \cap\left\{y \mid d\left(y, \partial S_{2}\right) \leq s^{2}\right\}} u_{1}^{\varepsilon}(x) u_{2}^{\varepsilon}(y) \mathrm{d} y \mathrm{~d} x+o(1) \\
& \leq \frac{1}{\varepsilon^{2}} \int_{x \in D_{h, s}} \int_{y \in E_{h+c s, s}} u_{1}^{\varepsilon}(x) u_{2}^{\varepsilon}(y) \mathrm{d} y \mathrm{~d} x+o(1) \\
& \leq \frac{1}{\varepsilon^{2}} \int_{y \in E_{h+c s, s}} \int_{x \in B_{1}(y)} 70 u_{1}^{\varepsilon}(x) u_{2}^{\varepsilon}(y) \mathrm{d} x \mathrm{~d} y+o(1) \\
& =\int_{E_{h+c s}} \Delta u_{2}^{\varepsilon}(y) d y+o(1)
\end{aligned}
$$


Similar computations give

$$
\int_{E_{h}} \Delta u_{2}^{\varepsilon}(y) d y \leq \int_{D_{h+c s}} \Delta u_{1}^{\varepsilon}(x) d x+o(1) .
$$

Letting first $\varepsilon$ and then $s$ go to 0 , the conclusion of the lemma follows.

Lemma 9.4. Under the assumptions of Theorem 9.2, let $\Gamma_{h}^{1}=\partial S_{1} \cap B_{h}(0)$ and let $\Gamma_{h}^{2}=$ $\left\{x+\nu(x): x \in \Gamma_{h}^{1}\right\}$. Then we have the limits

$$
\lim _{h \rightarrow 0} \frac{\int_{\Gamma_{h}^{2}} d A}{\int_{\Gamma_{h}^{1}} d A}=\prod_{\substack{i=1 \\ \varkappa_{i}(0) \neq 0}}^{n-1} \frac{\varkappa_{i}(0)}{\varkappa_{i}\left(e_{n}\right)} \quad \text { if } \varkappa_{i}(0) \neq 0 \text { for some } i=1, \ldots, n-1 \text {, }
$$

and

$$
\lim _{h \rightarrow 0} \frac{\int_{\Gamma_{h}^{2}} d A}{\int_{\Gamma_{h}^{1}} d A}=1 \quad \text { if } \varkappa_{i}(0)=0 \text { for any } i=1, \ldots, n-1 .
$$

Proof. Consider the diffeomorphism $f_{t}(x)=f(x, t)=x+t \nu(x)$. Then $\Gamma_{h}^{2}=f_{1}\left(\Gamma_{h}^{1}\right)$ and

$$
\int_{\Gamma_{h}^{2}} d A=\int_{\Gamma_{h}^{1}}\left|J f_{1}(x)\right| d A
$$

where $\left|J f_{1}\right|$ is the determinant of the Jacobian of $f_{1}$. Taking as basis of the tangent space at 0 the principal directions, $\tau_{i}$, then the differential of $f_{1}$ at $x$ is given by

$$
\left(d f_{1}\right)\left(\tau_{i}\right)=\tau_{i}+(d \nu)\left(\tau_{i}\right)=\tau_{i}-\varkappa_{i} \tau_{i}
$$

So,

$$
\left|J f_{1}(x)\right|=\prod_{i=1}^{n-1}\left(1-\varkappa_{i}(x)\right)
$$

and

$$
\frac{\int_{\Gamma_{h}^{2}} d A}{\int_{\Gamma_{h}^{1}} d A}=\frac{1}{\text { Area }\left(\Gamma_{h}^{1}\right)} \int_{\Gamma_{h}^{1}} \prod_{i=1}^{n-1}\left(1-\varkappa_{i}(x)\right) d A .
$$

Passing to the limit when $\mathrm{h}$ converges to zero, we obtain

$$
\lim _{h \rightarrow 0} \frac{\int_{\Gamma_{h}^{2}} d A}{\int_{\Gamma_{h}^{1}} d A}=\prod_{i=1}^{n-1}\left(1-\varkappa_{i}(0)\right) .
$$

Now, if $\varkappa_{i}(0) \neq 0$ for some $i=1, \ldots, n-1$, then

$$
\prod_{i=1}^{n-1}\left(1-\varkappa_{i}(0)\right)=\prod_{\substack{i=1 \\ \varkappa_{i}(0) \neq 0}}^{n-1}\left(1-\varkappa_{i}(0)\right)=\prod_{\substack{i=1 \\ \varkappa_{i}(0) \neq 0}}^{n-1}\left(\frac{1-\varkappa_{i}(0)}{\varkappa_{i}(0)} \varkappa_{i}(0)\right)=\prod_{\substack{i=1 \\ \varkappa_{i}(0) \neq 0}}^{n-1} \frac{\varkappa_{i}(0)}{\varkappa_{i}\left(e_{n}\right)},
$$


and $(9.6)$ follows.

$$
\begin{aligned}
& \text { If } \varkappa_{i}(0)=0 \text { for any } i=1, \ldots, n-1 \text {, then } \\
& \qquad \prod_{i=1}^{n-1}\left(1-\varkappa_{i}(0)\right)=1
\end{aligned}
$$

and we get 9.7 .

Proof of Theorem 9.2.

Let $\Gamma_{h}^{1}=\partial S_{1} \cap D_{h}$ and $\Gamma_{h}^{2}=\partial S_{2} \cap E_{h}$. The Laplacians $\Delta u_{i}$, are jump measures along $\partial S_{i}$, $i=1,2$, and satisfy

$$
\int_{D_{h}} \Delta u_{1}=-\int_{\Gamma_{h}^{1}} u_{\nu}^{1} d A \text { and } \int_{E_{h}} \Delta u_{2}=-\int_{\Gamma_{h}^{2}} u_{\nu}^{2} d A
$$

Then, using Lemma 9.3 we get

$$
1=\frac{\int_{D_{h}} \Delta u_{1}}{\int_{E_{h}} \Delta u_{2}}=\frac{\int_{\Gamma_{h}^{1}} u_{\nu}^{1} d A}{\int_{\Gamma_{h}^{2}} u_{\nu}^{2} d A},
$$

and so

$$
\frac{\int_{\Gamma_{h}^{1}} u_{\nu}^{1} d A}{\oint_{\Gamma_{h}^{2}} u_{\nu}^{2} d A}=\frac{\int_{\Gamma_{h}^{2}} d A}{\int_{\Gamma_{h}^{1}} d A} .
$$

Since, when $h \rightarrow 0$,

$$
\frac{\oint_{\Gamma_{h}^{1}} u_{\nu}^{1} d A}{\oint_{\Gamma_{h}^{2}} u_{\nu}^{2} d A} \rightarrow \frac{u_{\nu}^{1}(0)}{u_{\nu}^{2}\left(e_{n}\right)},
$$

by Lemma 9.4 the conclusion of Theorem 9.2 follows.

\section{REFERENCES}

[1] L. Ambrosio, N. Fusco, and D. Pallara. Functions of bounded variation and free discontinuity problems. Oxford university press, 2000.

[2] L. Caffarelli. The obstacle problem revisited. Journal of Fourier Analysis and Applications, 4:383-402, 1998.

[3] L. Caffarelli and X. Cabré. Fully nonlinear elliptic equations, volume 43. American Mathematical Society, 1995.

[4] L. Caffarelli, A. L. Karakhanyan, and F.-H. Lin. The geometry of solutions to a segregation problem for nondivergence systems. Journal of Fixed Point Theory and Applications, 5:319-351, 2009.

[5] L. Caffarelli and F.-H. Lin. Singularly perturbed elliptic systems and multi-valued harmonic functions with free boundaries. Journal of the American Mathematical Society, 21:847-862, 2008.

[6] L. Caffarelli, P. A. Markowich, and J.-F. Pietschmann. On a price formation free boundary model by lasry and lions. Comptes Rendus Mathematique, 349(11):621-624, 2011. 
[7] G.-Q. Chen, W. P Ziemer, and M. Torres. Gauss-green theorem for weakly differentiable vector fields, sets of finite perimeter, and balance laws. Communications on Pure and Applied Mathematics, 62(2):242-304, 2009.

[8] M. Conti and V. Felli. Coexistence and segregation for strongly competing species in special domains. Interfaces and Free Boundaries, 10(2):173-195, 2008.

[9] M. Conti and V. Felli. Minimal coexistence configurations for multispecies systems. Nonlinear Analysis: Theory, Methods 8 Applications, 71(7):3163-3175, 2009.

[10] M. Conti and V. Felli. Global minimizers of coexistence for competing species. Journal of the London Mathematical Society. Second Series, 83(3):606 618, 2011.

[11] M. Conti, S. Terracini, and G. Verzini. Nehari's problem and competing species systems. Ann. I. H. Poincare, 19:871-888, 2002.

[12] M. Conti, S. Terracini, and G. Verzini. Asymptotic estimates for the spatial segregation of competitive systems. Advances in Mathematics, 195:524-560, 2005.

[13] M. Conti, S. Terracini, and G. Verzini. A variational problem for the spatial segregation of reaction-diffusion systems. Indiana University mathematics journal, 54(3):779-815, 2005.

[14] M. Conti, G. Verzini, and S. Terracini. A regularity theory for optimal partition problems. In SPT 2004 Symmetry and perturbation theory, pages 91-98. World Sci. Publ., Hackensack, NJ, 2005.

[15] J. H. Cushman, G. D. Martinsen, and A. I. Mazeroll. Density-and size-dependent spacing of ant nests: evidence for intraspecific competition. Oecologia, 77(4):522-525, 1988.

[16] E. N. Dancer. Competing species systems with diffusion and large interactions. Rendiconti del Seminario Matematico e Fisico di Milano, 65:2333 (1997), 1995.

[17] E. N. Dancer and Y. Du. Positive solutions for a three-species competition system with diffusion i. general existence results. Nonlinear Analysis. Theory, Methods, Appl. An International Multidisciplinary Journal. Series A: Theory and Methods, 24(3):337-357, 1995.

[18] E. N. Dancer and Y. Du. Positive solutions for a three-species competition system with diffusion ii.the case of equal birth rates. Nonlinear Analysis. Theory, Methods, Appl. An International Multidisciplinary Journal. Series A: Theory and Methods, 24(3):359-373, 1995.

[19] E. N. Dancer and Yihong Du. On a free boundary problem arising from population biology. Indiana University Mathematics Journal, 52(1):5167, 2003.

[20] E. N. Dancer, D. Hilhorst, M. Mimura, and L. A. Peletier. Spatial segregation limit of a competition-diffusion system. European Journal of Applied Mathematics, 10(2):97115, 1999.

[21] E. N. Dancer and Z. Wang, K.and Zhang. The limit equation for the gross-pitaevskii equations and s. terracini's conjecture. Journal of Functional Analysis, 262(3):1087-1131, 2012.

[22] L. C. Evans and R. F. Gariepy. Measure theory and fine properties of functions. CRC press, 2015.

[23] D. Gilbarg and N.S. Trudinger. Elliptic partial differential equations of second order, volume 224. Springer Verlag, 2001.

[24] H. Ishii. On the equivalence of two notions of weak solutions, viscosity solutions and distribution solutions. Funkcial. Ekvac, 38(1):101-120, 1995.

[25] J.-M. Lasry and P.-L. Lions. Mean field games. Japanese Journal of Mathematics, 2(1):229-260, 2007.

[26] M. Mimura, S.-I. Ei, and Q. Fang. Effect of domain-shape on coexistence problems in a competition-diffusion system. J. Math. Biol., pages 219-237, 1991.

[27] B. Noris, S. Terracini, H. Tavares, and G. Verzini. Uniform hölder bounds for nonlinear schrödinger systems with strong competition. Communications on pure and applied mathematics, 63(3):267-302, 2010.

[28] K. Ohsawa, K. Kawasaki, F. Takasu, and N. Shigesada. How does spatio-temporal disturbance influence species diversity in a hierarchical competitive system? prospective order of species coexistence and extinction. Popul Ecol, 45:239-247, 2003.

[29] R. T. Paine. Ecological determinism in the competition for space: The robert h. macarthur award lecture. Ecology, 65(5):1339-1348, 1984.

[30] V. Quitalo. A free boundary problem arising from segregation of populations with high competition. Archive for Rational Mechanics and Analysis, 210(3):857-908, 2013.

[31] N. Shigesada, K. Kawasaki, and E. Teramoto. The effects of interference competition on stability, structure and invasion of a multi-species system. Journal of Mathematical Biology, 21:97-113, 1984.

[32] N. Soave and A. Zilio. Uniform bounds for strongly competing systems: the optimal lipschitz case. Archive for Rational Mechanics and Analysis, 218(2):647-697, 2015. 
[33] J. Wei and T. Weth. Asymptotic behaviour of solutions of planar elliptic systems with strong competition. Nonlinearity, 21(2):305, 2008.

The University of Texas at Austin, Department of Mathematics - RLM 8.100, 2515 Speedway Stop C1200, Austin, TX 78712-1202

E-mail address: caffarel@math.utexas.edu

The University of Texas at Austin, Department of Mathematics - RLM 8.100, 2515 Speedway Stop C1200, Austin, TX 78712-1202

E-mail address: spatrizi@math.utexas.edu

Purdue University, Department of Mathematics, 150 N. University Street, West Lafayette, IN 47907-2067

E-mail address: vquitalo@math.purdue.edu 